Aus dem Institut für Neuropathologie (Prof. Dr. med. W. Brück)

der Medizinischen Fakultät der Universität Göttingen

\title{
Identification of Prognostically Relevant Cellular Markers of Differentiation in Glioblastoma
}

\author{
INAUGURAL - DISSERTATION \\ zur Erlangung des Doktorgrades \\ der Medizinischen Fakultät der \\ Georg-August-Universität zu Göttingen
}

vorgelegt von

Felix Behling

aus

Greven

Göttingen den 29.05.2015 
Identification of Prognostically Relevant Cellular Markers of Differentiation in Glioblastoma 
Dekan:

I. Berichterstatterin:

II. Berichterstatter/in:

III. Berichterstatter/in:

Tag der mündlichen Prüfung:
Prof. Dr. rer. nat. H.K. Kroemer

Prof. Dr. med. C. Stadelmann-Nessler 
Identification of Prognostically Relevant Cellular Markers of Differentiation in Glioblastoma 


\section{Contents}

List of Figures $\quad$ v

List of Tables $\quad$ ix

Abbreviations $\quad$ xi

1 Introduction 1

1.1 Glioblastoma Multiforme . . . . . . . . . . . . . . . . . . . 1

1.1.1 Epidemiology . . . . . . . . . . . . . . . . . . 1

1.1.2 Pathology . . . . . . . . . . . . . . . . . 2

1.1.3 Clinical Presentation and Diagnosis . . . . . . . . . . 5

1.1.4 Current Treatment . . . . . . . . . . . . . . . . . 8

1.2 Prognostic Markers . . . . . . . . . . . . . . . . . . . . . . . . 12

1.2.1 Clinical Factors . . . . . . . . . . . . . . . . . . 12

1.2.2 The Cancer Genome Atlas . . . . . . . . . . . . . . . . . . 12

1.2.3 MGMT Promoter Methylation . . . . . . . . . . . . . . . 13

1.2.4 Primary and Secondary Glioblastoma . . . . . . . . . . . . . . 14

1.2 .5 IDH-1 Mutation . . . . . . . . . . . . . . . . . . . . 14

1.2.6 GBMO, 1p19q Co-deletion, NogoA and OLIG2 . . . . . . 15

$1.2 .7 \mathrm{Ki} 67 \ldots \ldots \ldots \ldots \ldots$

$1.2 .8 \quad \mathrm{P} 53 \ldots \ldots \ldots \ldots \ldots$

1.2 .9 GFAP . . . . . . . . . . . . . . . . 19

1.3 Aims of this Dissertation . . . . . . . . . . . . . . . 20

2 Materials and Methods $\quad 21$

2.1 Patient Collective . . . . . . . . . . . . . . . . . . . . . 21

2.2 Biospecimen Selection . . . . . . . . . . . . . . . . . . . 22

2.3 Tissue Microarray (TMA) . . . . . . . . . . . . . . . . . 23

2.3.1 Arrangement of Samples and Controls . . . . . . . . . . . 23

2.3.2 TMA Procedure . . . . . . . . . . . . . . . . . 24

2.4 Microtomy and Paraffin Section Preparation . . . . . . . . . . . . 26 
2.5 HE Staining . . . . . . . . . . . . . . . . . . . . . 26

2.6 Immunohistochemistry . . . . . . . . . . . . . . . . . 27

2.7 Microscopic Evaluation . . . . . . . . . . . . . . . . . 32

2.8 Quantification of Expression Levels . . . . . . . . . . . . . . . . . 32

2.9 Statistical Analysis . . . . . . . . . . . . . . . . . . . . . 33

3 Results 35

3.1 Patient Cohort Characterization . . . . . . . . . . . . . . . 35

3.2 Prognostic Impact of Clinical Factors . . . . . . . . . . . . . . . . . . 37

3.2 .1 Sex . . . . . . . . . . . . . . . . . . 37

3.2 .2 Age at Diagnosis . . . . . . . . . . . . . . . . 38

$3.2 .3 \mathrm{KPS} \ldots \ldots \ldots \ldots \ldots \ldots \ldots$

3.2.4 Extent of Resection . . . . . . . . . . . . . . . . . 43

3.2.5 Chemotherapy . . . . . . . . . . . . . . . . . 45

3.3 Prognostic Role of Immunohistochemical

Markers . . . . . . . . . . . . . . . . . 47

3.3 .1 IDH-1 Mutation . . . . . . . . . . . . . . . . . 47

3.3 .2 NogoA . . . . . . . . . . . . . . . 50

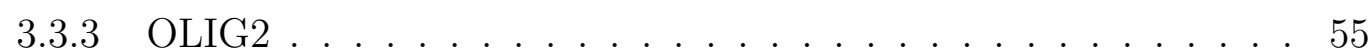

3.3 .4 Ki67 . . . . . . . . . . . . . . . . 63

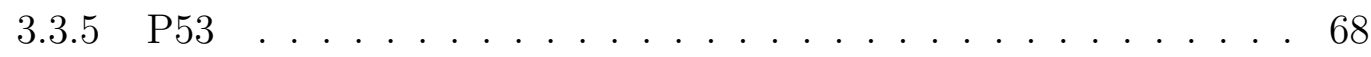

3.3.6 GFAP . . . . . . . . . . . . . . 75

3.4 Multivariate Analysis . . . . . . . . . . . . . . . . . . . 80

4 Discussion $\quad 81$

4.1 The Patient Collective . . . . . . . . . . . . . . . . . . . . 81

4.2 The Prognostic Impact of Clinical Factors . . . . . . . . . . . . . . . . 82

4.2 .1 Sex . . . . . . . . . . . . . . . . . 82

4.2 .2 Age and KPS . . . . . . . . . . . . . . . . . . . 82

4.2 .3 Extent of Resection . . . . . . . . . . . . . . . . . . . 83

4.2.4 Chemotherapy . . . . . . . . . . . . . . . . . . . 84

4.3 Prognostic Role of Immunohistochemical

Markers ....................... . . 85

4.3 .1 IDH-1 Mutation . . . . . . . . . . . . . . 85

4.3 .2 NogoA . . . . . . . . . . . . . . . . . 87

4.3 .3 OLIG2 . . . . . . . . . . . . . . . . . 88

4.3 .4 Ki67 . . . . . . . . . . . . . . . . . 90

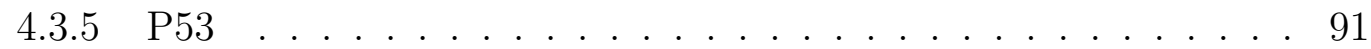

4.3 .6 GFAP . . . . . . . . . . . . . . . . 92 
Identification of Prognostically Relevant Cellular Markers of Differentiation in Glioblastoma

4.3.7 Multivariate Analysis . . . . . . . . . . . . . . . . . . . 93

$\begin{array}{lll}5 & \text { Summary } & 95\end{array}$

$\begin{array}{llr}6 & \text { Bibliography } & 97\end{array}$

$\begin{array}{lr}\text { Acknowledgements } & 119\end{array}$

$\begin{array}{ll}\text { Curriculum Vitae } & 121\end{array}$

CONTENTS iii 
Identification of Prognostically Relevant Cellular Markers of Differentiation in Glioblastoma 


\section{List of Figures}

1.1 Tumor necrosis and neovascularization in HE sections of GBM samples. . . . . . . . . . . . . . . . . . 3

2.1 Microcopic exclusion criteria. . . . . . . . . . . . . . 22

2.2 Area suitable for TMA. . . . . . . . . . . . . . . . . 23

2.3 Illustration of the TMA procedure. . . . . . . . . . . . . . 24

2.4 Completed TMA-block right before microtomy. . . . . . . . . . 25

2.5 Indirect IHC and the avidin-biotin complex method. . . . . . . 28

3.1 Distribution of overall survival within the collective of $\mathbf{1 2 0}$ GBM patients. . . . . . . . . . . . . 36

3.2 Distribution of age and KPS at the time of diagnosis of glioblastoma. . . . . . . . . . . . . . . . 36

3.3 No influence of sex on overall survival in GBM. . . . . . . . . . 38

3.4 Younger age is a significant prognostic factor in GBM I. . . . . 39

3.5 Younger age is a significant prognostic factor in GBM II. . . . 40

3.6 Higher Karnofsky scores are associated with longer overall survival in GBM I. . . . . . . . . . . . . . . . . . . . . . . . . . 41

3.7 Higher Karnofsky scores are associated with longer overall survival in GBM II. . . . . . . . . . . . . . . . . . . . . . . . . . . . 42

3.8 Higher Karnofsky scores are associated with longer overall survival in GBM III. . . . . . . . . . . . . . . . . . . . . . . . . . 43

3.9 Gross total resection shows a tendency towards longer overall survival. . . . . . . . . . . . . . . . . . . . . . 44

3.10 No differential prognostic impact among alkylating chemotherapeutic agents in GBM. . . . . . . . . . . . . . . . . . . 46

3.11 IDH-1 mutation controls. . . . . . . . . . . . . . . . . . . . . . . 48

3.12 IDH-1 mutations in GBM. . . . . . . . . . . . . . . . . . . . . . . 48

3.13 Trend towards younger age of patients with IDH-1 mutations in GBM. . . . . . . . . . . . . . . . . . . . . . . . . . . . . . . . . . . 49

3.14 NogoA controls. . . . . . . . . . . . . . . . . 50 
3.15 Quantification of NogoA immunopositivity. . . . . . . . . . . 51

3.16 Distribution of NogoA immunopositivity. . . . . . . . . . . . . 51

3.17 NogoA cutoff at +/- without significant differences. . . . . . . . 52

3.18 NogoA cutoff at + without significant differences. . . . . . . . . 53

3.19 NogoA cutoff at ++ without significant differences. . . . . . . . 54

3.20 OLIG2 controls. . . . . . . . . . . . . . . . . . . 55

3.21 Examples of different OLIG2 expression levels. . . . . . . . . . 56

3.22 Distribution of OLIG2 expression throughout the sample collective. . . . . . . . . . . . . . . . . 5 56

3.23 Longer mean overall survival of OLIG2 expression below $5 \%$ failed statistical significance. . . . . . . . . . . . . . 57

3.24 Longer mean overall survival of OLIG2 expression below $10 \%$ failed statistical significance. . . . . . . . . . . . . . . . 58

3.25 Longer mean overall survival of OLIG2 expression below $20 \%$ failed statistical significance. . . . . . . . . . . . . . . . 59

3.26 OLIG 2 expression below $\mathbf{3 0 \%}$ shows a statistical trend towards longer overall survival. . . . . . . . . . . . . . . . . . . . . 60

3.27 OLIG2 cutoff at $\mathbf{4 0 \%}$ without significant differences. . . . . . . 61

3.28 OLIG 2 cutoff at $\mathbf{5 0 \%}$ without significant differences. . . . . . . 62

3.29 Ki67 controls. . . . . . . . . . . . . . . . . . . . . . . . 63

3.30 Examples of differing Ki67 expression levels. . . . . . . . . . . . 64

3.31 Distribution of Ki67 expression rates among GBMs. . . . . . . 64

$3.32 \mathrm{Ki67}$ cutoff at $\mathbf{5 \%}$ without significant differences. . . . . . . . . 65

$3.33 \mathrm{Ki67}$ cutoff at $\mathbf{1 0 \%}$ without significant differences. . . . . . . . . 66

$3.34 \mathrm{Ki67}$ cutoff at $\mathbf{2 0 \%}$ without significant differences. . . . . . . . . 67

3.35 P53 controls. . . . . . . . . . . . . . . . . . . . 68

3.36 Examples of differing p53 immunopositivity rates. . . . . . . . . 69

3.37 Distribution of p53 immunopositivity rates among glioblas-

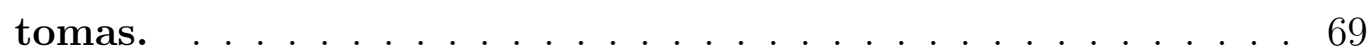

3.38 GBMs with p53 positive tumor cells have a significantly longer overall survival while being younger and of better clinical status. 70

3.39 P53 cutoff at 5\% without significant differences. . . . . . . . . . 71

3.40 P53 cutoff at $10 \%$ without significant differences. . . . . . . . . 72

3.41 Patients with p53 immunopositivity rates above $20 \%$ showed a tendency towards younger age. . . . . . . . . . . . 73

3.42 Patients with p53 immunopositivity rates above $30 \%$ showed a tendency towards longer overall survival. . . . . . . . . . . . 74

3.43 GFAP controls. . . . . . . . . . . . . . . . . . . . . . . 75

3.44 Quantification of GFAP immunopositivity. . . . . . . . . . . . 76 
Identification of Prognostically Relevant Cellular Markers of Differentiation in Glioblastoma

3.45 A GFAP score below + showed a tendency towards younger age. . . . . . . . . . . . . . . . . 77

3.46 GFAP cutoff at $+/++$ without significant differences. . . . . 78

3.47 GFAP cutoff at ++ without significant differences. . . . . . . . 79 
Identification of Prognostically Relevant Cellular Markers of Differentiation in Glioblastoma 


\section{List of Tables}

1.1 Presenting symptoms of patients suffering from glioblastoma multiforme (according to Chang and Parney 2005). . . . . . . 5

1.2 Karnofsky performance status scale (KPS) (according to Greenberg 2010). . . . . . . . . . . . . . . . . . . . . . . . . . . . . . 6

2.1 Materials used for Tissue Microarray and microtomy. . . . . . . 25

2.2 Materials used for HE staining. . . . . . . . . . . . . . . . . . 27

2.3 Materials used for immunohistochemistry. . . . . . . . . . . 31

2.4 Antibodies used for immunohistochemical staining. . . . . . . . 32

2.5 Computer Software used in this study. . . . . . . . . . . . . . 33

3.1 Results of the Cox regression analysis. . . . . . . . . . . . . 80 
Identification of Prognostically Relevant Cellular Markers of Differentiation in Glioblastoma 


\section{Abbreviations}

$\begin{array}{ll}\text { 1p } & \text { Small arm of chromosome 1 } \\ \text { 19q } & \text { Long arm of chromosome 19 } \\ \text { 5-ALA } & \text { 5-Aminolevulinic acid } \\ \text { A } & \text { Adenine } \\ \text { AED } & \text { Antiepileptic drug } \\ \text { AQP } & \text { Aquaporin } \\ \text { BCNU } & \text { Bis-chloroethylnitrosurea } \\ \text { BTRE } & \text { Brain tumor related epilepsy } \\ \text { C } & \text { Cytosine } \\ \text { CT } & \text { Computed tomography } \\ \text { DAB } & \text { 3,3'-diaminobenzidine } \\ \text { ddTMZ } & \text { Dense dose temozolomide } \\ \text { DNA } & \text { Deoxyribonucleic acid } \\ \text { EBRT } & \text { External beam radiation therapy } \\ \text { EDTA } & \text { Ethylenediaminetetraacetic acid } \\ \text { Fab } & \text { Fragment, antigen binding } \\ \text { FDA } & \text { Food and Drug Administration } \\ \text { EGFR } & \text { Epidermal growth factor receptor } \\ \text { FCS } & \text { Fetal calf serum } \\ \text { FGS } & \text { Fluorescence guided surgery } \\ \text { G } & \text { Guanine } \\ \text { GBM } & \text { Glioblastoma multiforme } \\ \text { GBMO } & \text { GBM with oligodendroglioma component } \\ \text { GC } & \text { Giant cell glioblastoma } \\ \text { GKS } & \text { Gamma knife surgery } \\ \text { GTR } & \text { Gross total resection } \\ \text { Gy } & \text { Gray (energy of ionizing radiation per kilogram of matter) } \\ \text { HDR-BRT } & \text { High dose rate brachytherapy } \\ \text { HE section } & \text { Hematoxylin and eosin section } \\ \text { HSP990 } & \text { Heat shock protein 990 } \\ & \end{array}$




\begin{tabular}{|c|c|}
\hline $\mathrm{IDH}$ & Isocitrate dehydrogenase \\
\hline $\mathrm{IHC}$ & Immunohistochemistry \\
\hline ISM & Intraoperative stimulation mapping \\
\hline KPS & Karnofsky performance status scale \\
\hline LMWH & Low molecular weight heparin \\
\hline $\mathrm{LOH}$ & Loss of heterozygosity \\
\hline MGMT & O-6-methylguanine-DNA-methyltransferase \\
\hline MMR & Mismatch repair enzyme \\
\hline $\mathrm{MP}$ & Microparticle \\
\hline MRA & Magnetic resonance angiography \\
\hline MRI & Magnetic resonance imaging \\
\hline MSH-6 & MutS homolog 6 \\
\hline NGS & Normal goat serum \\
\hline NogoA & Neurite outgrowth inhibitor A \\
\hline NVP-HSP990 & Oral inhibitor of HSP990 \\
\hline p16INK4A & Cyclin-dependent kinase inhibitor $2 \mathrm{~A}$ \\
\hline $\mathrm{p} 53$ & Protein 53 \\
\hline PBS & Phosphate buffered saline \\
\hline PDGFR & Platelet derived growth factor receptor \\
\hline PET & Positron emission tomography \\
\hline PGS & Primary gliosarcoma \\
\hline $\mathrm{PI}(3) \mathrm{K}$ & Phosphoinositide 3-kinase \\
\hline PTEN & Phosphatase and tensin homolog \\
\hline $\mathrm{R} 0$ & Complete tumor resection, no microscopic residues at resection margins \\
\hline RAS & Oncogene first described in rat sarcoma \\
\hline $\mathrm{RB}$ & Retinoblastoma protein \\
\hline RNA & Ribonucleic acid \\
\hline RTK & Receptor tyrosine kinase \\
\hline $\mathrm{T}$ & Thymine \\
\hline TBE & Tris/Borate/EDTA, a buffer solution \\
\hline TCGA & The Cancer Genome Atlas \\
\hline TMA & Tissue microarray \\
\hline TMS & Transcranial magnetic stimulation \\
\hline TP53 & Tumor protein 53 \\
\hline VEGF & Vascular endothelial growth factor \\
\hline VTE & Venous thromboembolism \\
\hline WHO & World Health Organization \\
\hline
\end{tabular}




\section{Chapter 1}

\section{Introduction}

\subsection{Glioblastoma Multiforme}

\subsubsection{Epidemiology}

Glioblastoma multiforme (GBM) is the most common malignant primary brain tumor in adults. It is also one of the most common primary brain tumors overall, ranking second after meningiomas. In the United States 3.19 individuals per 100,000 were diagnosed with GBM each year, between 2005 and 2009. An extrapolation reveals approximately 9,000 new cases per year (Dolecek et al. 2012). An earlier study conducted in Switzerland revealed a similar incidence of 3.32 and 2.24 per 100,000 individuals per year between 1980 and 1994, for male and female patients respectively (Ohgaki et al. 2004). Although any age group can develop this kind of tumor, the average American is 64 years old when diagnosed with GBM. The male to female incidence rate ratio is 1.58 . Glioblastoma is also the most aggressive primary brain tumor that holds the worst prognosis of all malignancies of the central nervous system. (Dolecek et al. 2012). There is no detailed data available on the specific epidemiology of malignant gliomas in Germany (Robert Koch Institiute 2012).

Despite great efforts in neurooncological research and a multimodal treatment approach, patients suffering from glioblastoma have a median survival of 14.6 months (Stupp et al. 2005). However, a subgroup of long-term survivors that are believed to constitute $3-5 \%$ of all glioblastomas, exceed an overall survival of 36 months (Krex et al. 2007). 


\subsubsection{Pathology}

The glioblastoma multiforme is a poorly differentiated tumor that is believed to arise from astrocytes, the most abundant glial cell of the nervous system (Golgi 1894, Koch 2007, Louis et al. 2007). Nevertheless, this context is questioned by the discovery of glioma stem cells, a primitive neural progenitor cell, which may be the source of gliomagenesis (Singh et al. 2004, Fan et al. 2007). Highly variable morphology due to the irregular presence and varying extent of necrosis, bleeding and cysts are characteristic of glioblastoma and led Percival Bailey and Harvey Cushing to coin the term multiforme (Bailey and Cushing 1926).

A crucial feature of theses tumors is the rapid diffusely infiltrative growth along white matter tracts (Scherer 1940a, Holland 2000). A common example is the so-called butterfly glioma which is the result of tumor infiltration into the opposite frontal lobe via tracts of the corpus callosum (Greenberg 2010). Not to be confused with invasive growth of glioma cells are multicentric gliomas, which present distinct lesions that cannot be attributed to tumor cell spread. They are believed to constitute 2.3 to 9.5 $\%$ of all glioblastomas (Barnard and Geddes 1987, Turola et al. 2009). In some cases glioma cells can also disseminate through the cerebrospinal fluid (Erlich and Davis 1979, Choucair et al. 1986). This is referred to as meningeal gliomatosis and can manifest as communicating hydrocephalus, cranial neuropathies or radiculopathies (Greenberg 2010). Extraneural metastases, on the other hand, are a very rare event in GBM (Choucair et al. 1986). Usually by the time of diagnosis, neoplastic cells already extend well beyond the area of enhancement seen on MRI scans (Holland 2000 ). In over $90 \%$ of operated malignant gliomas, invading glial tumor cells cause a quick recurrence within a few centimeters of the resection cavity (Giese et al. 2003, Gaspar et al. 1992, Burger et al. 1983, Hochberg and Pruitt 1980).

The identification of typical histopathological features is crucial for establishing the diagnosis of glioblastoma multiforme. According to the World Health Organization, astrocytomas are graded on the basis of these characteristic microscopic properties. The grading scheme was developed to predict clinical behavior. Pilocytic astrocytomas (WHO grad I) are non-malignant gliomas since they do not show infiltrative growth. Patients can be cured after complete surgical resection and pilocytic astrocytomas never progress to a higher grade (Jones et al. 2012). Furthermore, the grades II through IV describe the gradual changes that occur in gliomagenesis. The lowgrade diffuse astrocytomas (WHO grade II) exhibit merely a few histopathological changes like increased cellular density and pleomorphic astrocytic tumor cells. They have a relatively good prognosis. Anaplastic astrocytomas (WHO grade III) and GBMs (WHO grade IV) represent high-grade astrocytomas that are associated with decreased survival. Both tumors show diffuse infiltration of the surrounding brain 
tissue. In addition to the histological characeristics of low-grade gliomas, the presence of increased mitotic activity immediately leads to a higher grading and establishes the diagnosis of anaplastic astrocytoma (WHO grade III). The diagnosis of GBM (WHO grade IV) is made by the presence of a tumor necrosis or abnormal neovascularization (Louis et al 2007) (see Figure 1.1). Especially necroses with pseudopalisade formation have been linked to microvascular changes and are believed to play a role in accelerated tumor growth in glioblastoma (Rong et al. 2006, Brat and Van Meir 2004).

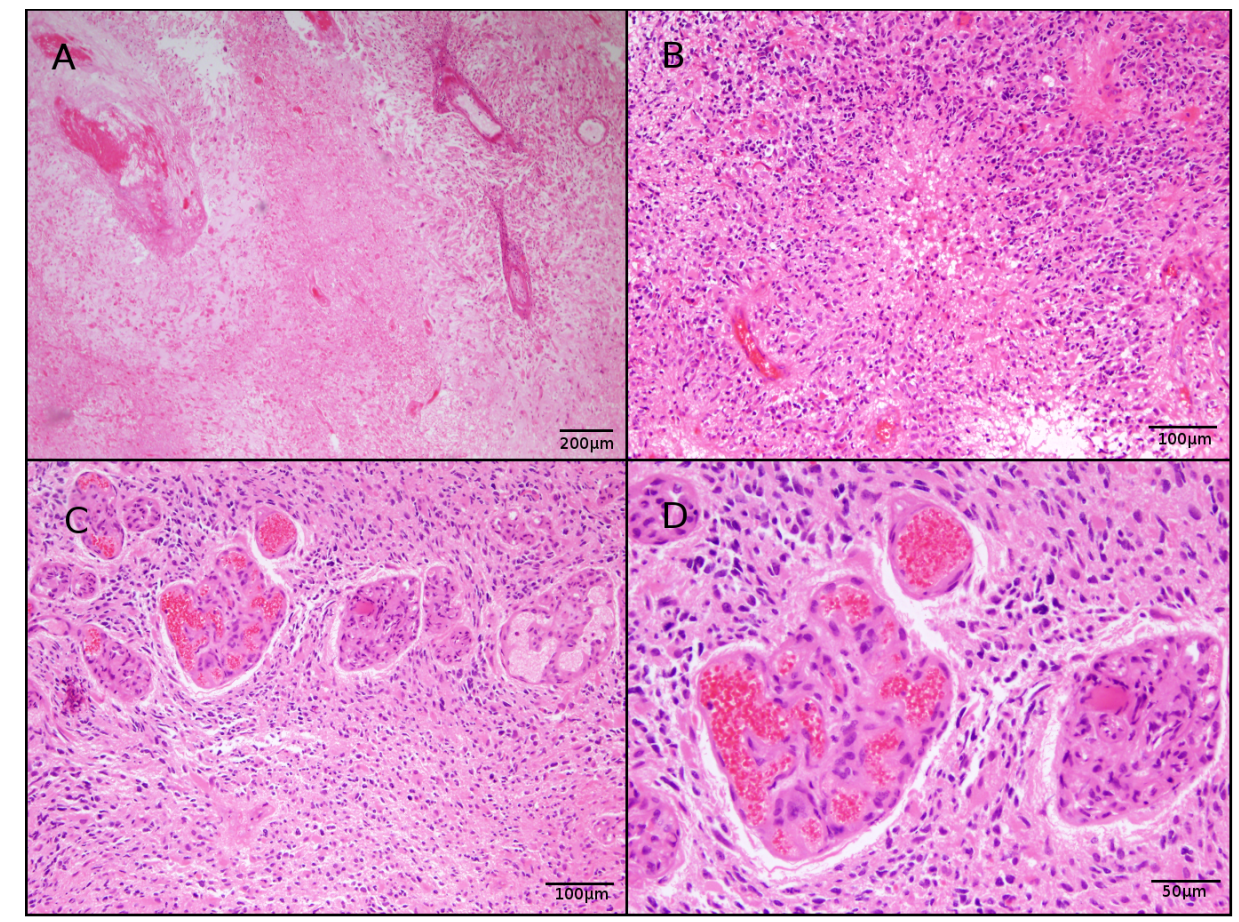

Figure 1.1: Tumor necrosis and neovascularization in HE sections of GBM samples. A: Area of extensive necrosis at 40-fold magnification. B: Typical tumor necrosis with pseudopalisade formation at 100-fold magnification. $\boldsymbol{C}$ and $\boldsymbol{D}$ : Neovascularizations at 100- and 200-fold magnification, respectively. Both, tumor necrosis and neovascularizaiton, are diagnostic histopathological features of Glioblastoma multiforme.

Two histopathological variants to the above mentioned GBM features like the primary gliosarcoma (PGS) and the giant cell glioblastoma (GC) exist. PGS is a rare variant of GBM with an incidence of 1.8-2.8\% of glioblastomas (Lutterbach et al. 2001). These tumors possess glial and metaplastic mesenchymal properties (Han et al. 2010, Miller and Perry 2007). There is some evidence of differing clinical features compared to GBMs. Temporal lobe predilection, common extracranial metastases and possible meningioma-like appearance on imaging scans and on gross pathological examination suggest gliosarcomas to represent a separate clinical entity (Han et al. 2010, Lutterbach et al. 2001, Maiuri et al. 1990, Smith et al. 1969). Due to low case numbers, a clear statement on a possible differing prognosis of gliosarcomas compared to glioblastomas could not be made yet (Han et al. 2010). The giant cell glioblastoma (GC) is another variant and accounts for 2-5\% of all GBMs (Kozac and Moody 2009). 
Its name is based on a characteristic histopathological feature, multinucleated giant cells (Louis et al. 2007). In contrast to GBM patients usually present at a younger age and have a slightly more favorable prognosis. This may be due to the fact that GCs are more likely to undergo complete resection and therefore generally receive a more aggressive treatment (Kozac and Moody 2009, Shinojima et al. 2004, Palma et al. 1989).

Less than $10 \%$ of GBMs are secondary glioblastomas that arise from lower grade gliomas and show differing clinical characteristics (Ohgaki and Kleihues 2013). They are outlined in detail in chapter 1.2.4.

Gliomas in general can occur in any region of the brain. A recent study showed that $86 \%$ of gliomas are located in the cerebral lobes with a preference for the frontal and temporal lobes of $40 \%$ and $29 \%$, respectively. With $14 \%$ parietal lobe gliomas are less common while occipital lesions are rare (3\%). Only $1.5 \%$ of all gliomas originate from the cerebellum and just $4.1 \%$ from the brainstem. The right hemisphere seems to be slightly more frequently affected than the left (Larjavaara et al. 2007). This was also observed in a prior study, but could not be linked to mobile phone use with the dominant hand as a cause of tumor localization (Ali Kahn et al. 2007).

Many potential environmental risk factors have been evaluated, but only the exposure to high-dose ionizing radiation could be proven as a true risk factor for the development of glioblastoma multiforme. Additionally, there are several genetic syndromes that are associated with an increased risk such as Li-Fraumeni syndrome, Von HippelLindau disease, multiple endocrine neoplasia type 1, neurofibromatosis type 1 and 2, tuberous sclerosis, Turcot's syndrome and nevoid basal cell carcinoma syndrome. Use of alcohol or tobacco, exposure to cellular phones or extremely low-frequency electromagnetic fields, infections, head injuries or the exposure to a variety of occupational chemicals still remain unproven in connection with gliomagenesis (Chandana et al. 2008, Fisher et al. 2006, Wrensch et al. 2002).

The cause of death of patients suffering from glioblastoma multiforme seems to be multifactorial. Autopsies of 117 patients revealed that many cases succumbed to more than one cause of death. Signs of herniation were the most common findings (61\%) but other possible lethal conditions could be revealed as well, such as neutroninduced cerebral injury, brain stem invasion, surgical complications such as bleeding and cerebral edema or severe systemic illnesses (Silbergeld et al. 1991). 


\subsubsection{Clinical Presentation and Diagnosis}

Approximately 70 percent of patients with glioblastoma multiforme are diagnosed within 6 months after the onset of symptoms (Frankel et al. 1958). In only $7 \%$ of cases symptoms have been present for over a year (Schneider et al. 2010, Busch 1963). Brain tumors in general can cause a wide variety of neurological disturbances. They can range from focal motor, sensory or visual deficits over focal or generalized seizures to personality changes, language deficits and cognitive dysfunctions (Chang and Parney 2005, Chandana et al. 2008). The symptoms depend rather on the localization of the lesion then on the histopathological type of the tumor (Newton 1994, Chandana et al. 2008, Lee et al. 2010, Schneider et al. 2010). As examples, lesions of the dominant hemisphere are more commonly associated with language deficits and cognitive dysfunctions and a tumor mass in the occipital lobe typically causes visual disturbances (Taphoorn and Klein 2004, Batchelor et al. 2012). In patients with highgrade gliomas headache is the most common complaint (Frankel et al. 1958, Roth and Elvidge 1960) and presents similar to a tension-type headache in most cases (Forsyth and Posner1993). A study including 565 patients suffering from primary malignant glioma (WHO grade III and IV) produced an overview of the frequency of presenting symptoms. Table 1.1 depicts the results for the 418 glioblastomas according to that study (Chang and Parney 2005).

Table 1.1: Presenting symptoms of patients suffering from glioblastoma multiforme (according to Chang and Parney 2005).

\begin{tabular}{lc}
\hline Symptom & Percentage of patients experiencing the symptom \\
\hline Headache & 57.3 \\
Memory loss & 39.2 \\
Cognitive changes & 38.8 \\
Language deficit & 36.2 \\
Motor deficit & 35.9 \\
Personality changes & 27.4 \\
Seizure & 23.5 \\
Visual problems & 21.2 \\
Changes in consciousness & 18.3 \\
Nausea/vomiting & 14.8 \\
Sensory deficit & 11.9 \\
Papilledema & 4.6 \\
Other & 18.5 \\
\hline
\end{tabular}

Signs of increased intracranial pressure such as headache, reduced consciousness, papilledema, nausea and vomiting indicate a lesion with a significant mass effect. This can be caused by the tumor itself or several tumor complications like bleeding, cerebral edema, occlusive hydrocephalus or rapid tumor growth (Schneider et al. 2010, Chandana et al. 2008, Greenberg 2010). If the intracranial hypertension further 
increases, life-threatening brain herniation can occur and typically presents with a decreased level of consciousness resulting in coma. Additionally, pupillary abnormalities, extensor posturing and the Cushing reflex, consisting of increased blood pressure, bradycardia and irregular respiration, are signs of brainstem compression (Schneider et al. 2010, Greenberg 2010, Fodstad 2006).

The Karnofsky Performance Status Scale (KPS) is a widely used measure to quantify and monitor the functioning of cancer patients in everyday life (Mor et al. 1984, Greenberg 2010) (see Table 1.2). Several studies showed that a KPS below 70 was associated with a shorter survival of patients with GBM (Lacroix et al. 2001, Laws et al. 2003, Habberstad et al. 2012). Besides taking a detailed patient history and conducting a physical examination, the KPS should be noted at the first patient contact and reassessed at every follow-up visit. Due to its prognostic value, it plays an important role in deciding further therapeutic strategies for the individual patient (Schneider et al. 2010).

Table 1.2: Karnofsky performance status scale (KPS) (according to Greenberg 2010).

\begin{tabular}{cl}
\hline KPS in percent & Performance status \\
\hline 100 & normal, no complaints, no signs of disease \\
90 & capable of normal activity, few symptoms or signs of disease \\
80 & normal activity with some difficulty, some symptoms or signs \\
70 & caring for self, not capable of normal activity or work \\
60 & requiring some help, can take care of most personal requirements \\
50 & requires help often, requires frequent medical care \\
40 & disabled, requires special care and help \\
30 & severely disabled, hospital admission indicated but no risk of death \\
20 & very ill, urgently requiring admission, requires supportive measures \\
& or treatment \\
10 & moribund, rapidly progressive fatal disease processes \\
0 & death \\
\hline
\end{tabular}

In order to exclude most non-neoplastic differential diagnoses, to attain information on the size and location of the lesion and to confirm the indication for surgery, a magnetic resonance imaging (MRI) scan of the brain should be the next diagnostic measure (Jenkinson et al. 2007). Furthermore, the high tissue contrast of an MRI scan allows more precise statements on secondary phenomena, such as mass effect, edema, hemorrhage and necrosis. A glioblastoma typically presents as a ring-enhancing lesion on a T1-weighted MRI or CT scan with contrast agent. Low grade gliomas, on the other hand, typically present as hypointense lesions on T1-weighted MRI with no or very little enhancement. In malignant gliomas the enhancement is based on leakage of contrast agent through the disrupted blood brain barrier and represents a hypercellu- 
lar region of the tumor (Jacob et al. 2005). However, glioblastoma tumor cells usually expand well beyond the area of enhancement (Holland 2000). The non-enhancing center of the lesion depicts an area of necrosis (Jacob et al. 2005). A hypointense region surrounding the ring-shaped enhancement can be found frequently on T1-weighted MRI scans of glioblastomas and describes parenchymal edema (Jacob et al. 2005). Studies revealed that peritumoral edema is associated with elevated expression levels of the vascular endothelial growth factor (VEGF) which increases the permeability of the blood brain barrier and causes the formation of neovascularizations (Seidel 2011, Carlson et al. 2007, Machein and Plate 2000, Strugar et al. 1995). A variety of additional scanning techniques can be utilized to asses other features of a brain tumor such as vascularization (magnetic resonance angiography (MRA)), diffusion and perfusion (diffusion and perfusion weighted imaging (DWI/PWI)), metabolic activity (positron emission tomography (PET)) or the risk of neurological deficits after surgical resection (functional MRI (fMRI) transcranial magnetic stimulation (TMS)) (Jacobs et al. 2005).

In order to assert the suspicion of a tumor of the central nervous system, a histopathological examination of the tumor according to the WHO classification is essential. Therefore a tumor biopsy or a tumor resection is necessary. Additionally to the classical characteristics of astrocytic tumors, any form of tumor necrosis or the presence of abnormal endothelial proliferations on histopathological examination confirms the diagnosis of GBM (as described in chapter 1.2) (Louis et al. 2007). 


\subsubsection{Current Treatment}

The standard of care for glioblastoma multiforme has not changed for many decades (Holland 2000) and involves the three common pillars of cancer treatment: surgery, radiotherapy and chemotherapy.

\section{Surgery}

Maximal surgical resection of the tumor mass is crucial to relieve symptoms caused by elevated intracranial pressure and significantly improves overall survival (Chandana et al. 2008, Sanai and Berger 2008, Simpson et al. 1993). The extent of resection necessary to improve overall survival has been assessed in several studies and ranges from 78 to 98\% (Yong and Lonser 2011, Sanai et al. 2011, Lacroix et al. 2001). Depending on the location of the tumor mass, more extensive resection increases the risk of surgically acquired neurological deficits, which in turn are associated with decreased survival (Yong and Lonser 2011, Gulati et al. 2011, McGirt 2009b). Therefore malignant gliomas, especially if located in eloquent areas, should undergo careful neurosurgical evaluation regarding resectability and the surgical risk of a possible extensive resection (Yong and Lonser 2011).

Based on its invasive nature, a complete resection ( $\mathrm{R} 0$ resection) of glioblastomas is not feasible (Schneider et al. 2010). Hence, the term complete resection merely describes the removal of all enhancing tumor mass seen on preoperative MRI scans often referred to as "gross total resection" (GTR). There are surgical approaches that intend to widen the extent of resection beyond this area. A technique that has become increasingly popular is fluorescence guided surgery (FGS). The prodrug 5-aminolevulinic acid (5-ALA) is administered to the patient several hours prior to surgery and accumulates in malignant glioblastoma cells as a porphyrin with fluorescent features. With the help of a special filter, that can be integrated into the operating microscope, infiltrating tumor cells outside the area of enhancement can be visualized intraoperatively and removed. Several studies suggest a significant increase in overall survival of patients undergoing FGS (Stummer et al. 2000, Stummer et al. 2008, Orzaiz 2013). Unfortunately, due to the location of some glioblastomas in eloquent areas, several cases are excluded from this aggressive surgical approach.

With the aid of intraoperative stimulation mapping (ISM), tumors in eloquent areas of the brain can undergo gross total resection while minimizing the risk of surgically acquired long-term neurological deficits (Hamer et al. 2012). In order to map brain regions of interest, the patient has to be awake and cooperative during a part of the surgery, which poses a challenge to all medical professionals involved in the perioperative management, especially to anesthesiologists (Erickson and Cole 2012, Conte et al. 2008). 


\section{Chemotherapy}

After gross total resection of the tumor mass, patients suffering from glioblastoma usually receive adjuvant chemotherapy with alkylating agents. In 2005 a randomized trial, including 573 patients, revealed an increased overall survival for individuals treated with temozolomide additionally to radiotherapy compared to radiotherapy alone (14.6 and 12.1 months respectively). Patients received temozolomide concomitantly to radiotherapy for 7 days a week, but not exceeding 49 days. After a 4 week break 6 cycles of additional temozolomide in a 28-day rhythm were administered, with each cycle lasting 5 days (Stupp et al. 2005). This treatment regimen soon evolved to the common standard of treatment for glioblastoma multiforme (Schneider et al. 2010, Chandana et al. 2008).

In general, systemic chemotherapy has several drawbacks. One is the questionable exposure of the tumor tissue to the therapeutic agent and another is the risk of toxic effects due to systemic application. To limit the effect of the chemotherapeutic agent to glioma cells, drug-impregnated biodegradable polymers have been developed. One example are the Gliadel ${ }^{\circledR}$ wafers that are placed on the surface of the resection cavity and ensure a high-dose delivery of carmustine (BCNU) over 2-3 weeks without major adverse effects. Especially patients with recurring glioblastomas, whose functional status would not allow another systemic chemotherapy, benefit from this novel therapy (Perry et al. 2007, Brem et al. 1995). Several studies also showed a positive effect on newly diagnosed GBMs, including evidence of efficacy of two phase III trials. (Hart 2011, Perry 2007, Westphal et al. 2003 and 2006, Valtonen et al. 1997). Furthermore, patients treated with radiotherapy and Gliadel ${ }^{\circledR}$ after primary tumor resection, have a significant benefit from additional temozolomide application without an increased risk of morbidity (McGirt et al. 2009a). In 2012, however, a retrospective study including 110 glioblastomas could not show a significant difference between Gliadel ${ }^{\circledR}$ implantation and standard chemotherapy (Catalan-Uribarrena et al. 2012). There is also evidence for an increased risk of adverse effects questioning the safety of the combination of local chemotherapy with standard adjuvant radiochemotherapy (Bock et al. 2010). Some authors question the cost-effectiveness of the application of BCNU wafers since conflicting data on the appropriate clinical use exist (Rogers et al. 2008, Garside et al. 2007).

Based on new insights into specific genetic aberrations of malignant gliomas, several targeted therapies have been developed. The goal is to block oncogenic pathways that were revealed to be major contributors to gliomagenesis and tumor progression. For example, the vascular endothelial growth factor (VEGF) is highly expressed in glioblastomas and is believed to play an important role in angiogenesis and tumor progression (Lamszus et al. 2003). Bevacizumab is a monoclonal antibody that tar- 
gets VEGF and consequently inhibits angiogenesis. As an additional treatment it has shown to improve the outcome of several cancerous diseases, like non-small-cell lung cancer, metastatic colorectal cancer and metastatic renal cancer (Sandler et al. 2006, Hurwitz et al. 2004, Yang et al. 2003). Bevacizumab has shown first promising results in glioblastomas and has recently been approved by the Food and Drug Administration (FDA) of the United States as a second-line treatment for GBM (Narayana 2012, Wick et al. 2011, Kreisl 2009, Cohen et al. 2009).

Many other targets, that were responsive to specific therapies in other cancerous diseases, have been identified to play major roles in the pathogenesis of glioblastoma. A great amount of research effort has been made to investigate these promising targets, like the epidermal growth factor receptor (EGFR) and the platelet derived growth factor receptor (PDGFR) (Wick et al. 2011). However, the expected clinical success, similar to first trials with bevacizumab, has not been accomplished yet. For example, a phase II trial on the efficacy of erlotinib, an inhibtor of the EGFR, was terminated due to unacceptable toxicity (Peereboom et al. 2010).

\section{Radiotherapy}

Simultaneously to adjuvant chemotherapy, postoperative external beam radiation therapy (EBRT) is initiated in most cases. A total dose of 50-60 Gy is delivered in fractions of 1.8-2 Gy on 5 days a week over approximately six weeks. The radiation dose is focused on tissue $2 \mathrm{~cm}$ beyond the resection cavity (Stupp et al. 2005, Laperriere et al. 2002).

An alternative method of radiotherapy is stereotactic radiosurgery. Two forms of this method are the gamma knife surgery (GKS) and the so-called CyberKnife. The patient receives a high-dose of radiation stereotactically targeted to the tumor, usually in a single session. Radiation damage to the surrounding tissue is kept to a minimum due to a rapid radiation fall-off. It is a possible treatment option for recurring glioblastomas (Romanelli et al. 2009, Crowley et al. 2006). But there is no clear benefit for newly diagnosed GBMs when compared to the current primary radiation regimen (Crowley et al. 2006, Souhami et al. 2004).

Another treatment option for recurrent GBM is high dose rate brachytherapy (HDR-BRT). Guided by CT under local anesthesia, several radiation sources are placed directly into the tumor mass (Chin et al. 1992). A recent study demonstrated a measurable benefit of HDR-BRT compared to re-resection alone or sole dense dose temozolomide chemotherapy (ddTMZ), regarding the overall survival of recurrent glioblastomas (Archavlis et al. 2013). 


\section{Symptomatic Support}

Vasogenic cerebral edema is common in brain tumors, especially in malignant gliomas. It can cause a significant mass effect and neurological deficits (Schneider et al. 2010). Some studies suggest that edema also plays a role in tumor cell migration, since, similar to invading glioma cells, interstitial fluid in vasogenic edema flows along white matter tracts (Geer and Grossman 1997). Various causes of peritumoral edema formation have been identified, such as endothelial abnormalities and vasoactive cytokines secreted by the tumor itself (Wick and Kueker 2004, Kalkanis et al. 1996, Shibata 1989). The positive effect of corticosteroids on tumor-associated cerebral edema has been known for several decades (Galicich 1961) and is a common supportive treatment for brain tumor patients with evidence of cerebral edema. Usually dexamethasone is applied, since it has less mineralocorticoid activity and is believed to have a lower risk of infections and cognitive impairment (Wick and Kueker 2004). In the light of wellknown side effects of systemic corticosteroid use, alternative treatments have been investigated. The phytotherapeutic agent H15, also known as boswellic acid, showed some promising results and could be used in the future to reduce steroid dosages (Kirste et al. 2011, Streffer et al. 2001).

Even though $20-40 \%$ of patients suffering from GBM experience seizures (Isoardo et al. 2012, Maschio 2012), a prophylactic administration of anticonvulsants is not recommended (Tremont-Lukats et al. 2008, Stevens 2006). Brain tumor related epilepsy (BTRE) is more common in low grade tumors (Chang and Parney 2005, Hildebrand et al. 2005) and most frequently presents as the focal type but also regularly shows secondary generalization (Maschio 2012). A wide range of antiepileptic drugs (AED) proved to be effective in controlling seizures in brain tumor patients Due to possible interference with the metabolizaiton of TMZ or other chemotherapeutic agents, older anticonvulsants, like phenytoin or valproic acid, should be avoided. Moreover, newer agents, like levetiracetam or lamotrigine, are also less likely to produce adverse effects (Kargiotis et al. 2011, Schneider et al. 2010).

Many cancerous diseases are associated with an increased risk of thromboembolic events (Horsted et al. 2012). Venous thromboembolism (VTE) is also a common complication of high grade gliomas (Perry 2012). Sartori et al. (2011) showed that glioblastomas have an increased procoagulant activity associated with elevated levels of circulating microparticles (MP). These MPs have been investigated in other malignancies and are believed to play a role in cancer-related VTEs (Thaler et al. 2012). Therefore low molecular weight heparin (LMWH) is used in several neurosurgical departments, even though it has not been approved for patients undergoing cerebral tumor operations due to the risk of postoperative bleeding (Schneider et al. 2010). This has only been surveyed in a few small scale studies (Hamilton et al. 1994). 


\subsection{Prognostic Markers}

\subsubsection{Clinical Factors}

A number of clinical factors that correlate with a better outcome for patients with glioblastoma multiforme have been described in the past. Patient age at diagnosis below 60 years, Karnofsky Performance Score (KPS) of at least 70\%, surgical resection exceeding $78-98 \%$ of the tumor mass seen on imaging scans, clinical presentation with seizures, frontal lobe localization and limited extent of tumor necrosis have all been found to be associated with longer survival (Lacroix et al. 2001, Laws et al. 2003, Yong and Lonser 2011, Sanai et al. 2011, Habberstad et al. 2012). There exist contradictory data suggesting a survival advantage for both sexes while other studies constitute no difference in overall survival (Scott et al. 1998, Caloglu et al. 2009, Verger et al. 2011, Johnson et al. 2012).

Especially patients with a poor functional status (low KPS) usually do not receive maximal treatment due to the risk of possible complications outweighing potential benefits (Marina et al. 2011, Villa et al. 1998, Baumann et al. 1994). Nonetheless, in 2011 Marina et al. reviewed treatment results of 74 patients with a KPS of $50 \%$ or lower at the time of diagnosis of GBM and presented data suggesting a benefit from radiotherapy and tumor resection. The role of chemotherapy within that patient group remained unclear (Marina et al. 2011). Advanced age is also a factor that used to withhold treatment from a subgroup of patients. But recently, several studies revealed a benefit of aggressive treatment despite the decreased overall survival of older patients (Weller and Wick 2011, Scott et al. 2011, Stupp et al. 2006, Roa et al. 2004, Chinot 2004). Thus, advanced age and poor functional status should not generally exclude patients from receiving appropriate treatment.

\subsubsection{The Cancer Genome Atlas}

In 2008, The Cancer Genome Atlas Research Network (TCGA) published openaccess data of a large-scale analysis of the most common mutations and their frequency in glioblastoma multiforme. Genetic aberrations of the p53, RB and the RTK/RAS/PI(3)K pathway were revealed to be key features that drive gliomagenesis in the majority of cases. Specifically, certain mutations were highlighted as possible therapeutic targets in the future (TCGA 2008).

In 2010, Verhaak et al. proclaimed the division of glioblastoma into 4 molecular subgroups based on gene expression profiles. It was divided into proneural, neural, mesenchymal and classical subtypes, which showed prognostic significance. $97 \%$ of tumors of the classical subtype held an amplification of the EGFR gene. A large portion of the mesenchymal subtype had a decreased expression of the NF-1 gene, 
which is known to be mutated in neurofibromatosis type 1. The proneural subtype was characterized by the combination of PDGFR-amplification and a point mutation in the IDH-1 gene and showed the least response to therapeutic approaches. The neural subtype simply displayed an increased expression of several neuronal markers, which can be found in normal brain tissue. However, there was no correlation between the subgroups and the MGMT-promoter methylation, an established prognostic marker in GBM (Verhaak et al. 2010).

\subsubsection{MGMT Promoter Methylation}

Over the last decade, several attempts have been made to establish a differentiation of glioblastomas into prognostic subtypes. One of the most important recent findings was the identification of the MGMT-promoter methylation in glioblastoma multiforme as a prognostic and predictive marker regarding the response to alkylating agents (Esteller et al. 2000, Hegi et al. 2005). After maximal treatment patients holding an MGMT-promoter methylation had an increased overall survival of 21.7 months compared to 12.7 months of patients receiving the same treatment but lacking the genetic aberration (Hegi et al. 2005). The data even suggested a benefit that was greater than most of the established clinical prognostic factors (Esteller et al. 2000). The hypermethylation of the promoter region silences the expression of the DNA repair enzyme O-6-methylguanine-DNA-methyltransferase (MGMT) (Watts et al. 1997). Consequently its function to restore guanine nucleosides that have been methylated by alkylating agents like temozolomide is impaired. The attached methyl group forces guanine to pair with thymine instead of cytosine, leading to a $\mathrm{C}: \mathrm{G}$ to A:T DNA-transition. This initiates several unsuccessful attempts at correction by mismatch repair enzymes which eventually result in impaired DNA synthesis and further lead to cytotoxicity by double strand breaks (Hegi et al. 2008, Yoshimoto et al. 2012). On the other hand The Cancer Genome Atlas (TCGA) study of 2008 noted that the application of temozolomide may lead to resistance towards alkylating agents. This is believed to be caused by an increased selection pressure in GBMs to attain mutations of the mismatch repair enzymes (MMR), which are responsible for the cytotoxic effect of alkylating agents (TCGA 2008). Loss of the mismatch repair enzyme MSH-6 has been described to correlate with resistance towards temozolomide treatment. Interestingly no genetic aberration of the MSH-6 gene was observed in the group of pretreatment glioblastomas (Cahill et al. 2007). It was also reported that a group of GBMs that were reassessed at recurrence, had lost their MGMT-promoter methylation status, which still had been present before initial treatment (Brandes et al. 2010). 


\subsubsection{Primary and Secondary Glioblastoma}

The concept of a possible existence of primary and secondary glioblastomas with significantly differing clinical behaviors was first suggested by Scherer (Scherer 1940). Over $90 \%$ of GBMs are primary and develop de novo, while less than $10 \%$ are secondary and emerge from preexisting precursor lesions such as low-grade diffuse or anaplastic gliomas. The latter subgroup usually has a longer overall survival rate. In contrast to glioblastomas in general, secondary GBMs typically occur in younger patients (mean age at diagnosis is 45 years compared to 64) and are more common in women (male to female ratio 0.65 compared to 1.58) (Dolecek et al. 2012, Ohgaki and Kleihues 2009, Ohgaki et al. 2004). A significant difference in the mutational profile of the two groups could be pointed out as well. Primary glioblastomas more commonly show epidermal growth factor receptor (EGFR) amplifications (approx. $40 \%$ ) and phosphatase and tensin homolog (PTEN) mutations (15-40\%), while both are quite rare in secondary GBMs (Ohgaki and Kleihues 2009, Ohgaki et al. 2004, Thoma et al. 1998, Watanabe et al. 1996, Ekstrand et al.1992). TP53 mutations, on the other hand, seem to be more frequent in secondary than in primary GBMs, with $63 \%$ compared to $28 \%$ respectively, and are already present in the lower-grade precursor lesions (Ohgaki and Kleihues 2009, Ohgaki and Kleihues 2004). Loss of heterozygosity $(\mathrm{LOH})$ on chromosome 10 seems to be a very common genetic aberration in both primary and secondary GBMs, with approximately $80 \%$ and $70 \%$ respectively (Ohgaki and Kleihues 2009, Ohgaki et al. 2004).

Mutations of the IDH-1 (isocitrate dehydrogenase 1) gene have been established as a reliable marker to differentiate secondary from primary glioblastomas and are further described in chapter 2.5. (Ohgaki and Kleihues 2013, Ohgaki and Kleihues 2009).

Recently, new insights into the regulation of lineage diversification of oligodendrocytes and astrocytes have been gained. Two transcription factors with antagonistic behaviour have been described. SOX10 and NFIA direct the diversification of astrocytes and oligodendrocytes and may play a role in the development of glioma subtypes (Glasgow et al. 2014).

\subsubsection{IDH-1 Mutation}

A prognostic factor that has been progressively established is the mutation of IDH-1. Five IDH genes code for three different enzymes, isocitrate dehydrogenase 1, 2 and 3. All three enzymes are involved in different pathways of the cellular metabolism ( $\mathrm{Yu}$ et al. 2010). A study in 2008 revealed that $12 \%$ of glioblastomas harbor an IDH-1 mutation. All mutations were observed at the amino acid R132, which serves as the substrate binding site. Such aberrations were mainly discovered in younger patients 
as well as secondary glioblastoma cases and were associated with longer overall survival (Parsons et al. 2008). Further investigations indicated the presence of IDH-1 mutations in approximately $70 \%$ of astrocytomas and oligodendrogliomas WHO grade II and III and secondary glioblastomas WHO grade IV. The mutation was associated with decreased activity of the gene product (isocitrate dehydrogenase 1) and, most importantly, with prolonged survival. Again, all mutations of IDH-1 occurred at the amino acid R132 (Yan et al. 2009). On average, patients suffering from anaplastic gliomas with IDH-1 mutations were 6 years younger at the time of diagnosis than their wildtype counterparts (Hartmann et al. 2009). IDH-1 mutations could also be detected in a small group of glioblastomas that were initially rated as primary GBMs, according to their clinical course. Likewise this group mainly consisted of younger patients with a prolonged overall survival. These cases were believed to have developed particularly fast from lower-grade gliomas, thus having led to incorrect initial assessment. This underlined the possible role of IDH-1 mutations in identifying secondary glioblastomas (Combs et al. 2011). IDH-2 mutations are less frequently found in glioma. Together with IDH-1 mutations they are mutually exclusive (Yan et al. 2009). In contrast to IDH-1 mutations, IDH-2 mutations can not be assessed via immunohistochemistry. Several studies analyzed the impact of IDH-1 and -2 mutations as a combined group (Zou et al. 2013).

In 2013, Ohgaki and Kleihues proclaimed the detection of an IDH-1 mutation as a definitive diagnostic marker for secondary glioblastoma multiforme, superior to clinical features (Ohgaki and Kleihues 2012).

\subsubsection{GBMO, 1p19q Co-deletion, NogoA and OLIG2}

Glioblastoma multiforme with an oligodendroglioma component (GBMO) represents a subgroup of GBM that seems to have differing genetic aberrations and clinical features. 1p19q co-deletion, MGMT-promoter methylation and IDH-1 mutations are more frequently detected in GBMOs than in GBMs. However, the oligodendroglial component does not provide a favorable outcome. But the identification of this subgroup has led to further investigations of a possible differing therapeutic management of GBMOs (Ha et al. 2013, Wang et al. 2012, Vordermark et al. 2006).

The presence of the co-deletion of $1 p$ and $19 q$ has been established as a positive prognostic marker in low- and high-grade oligodendrogliomas (Jenkins et al. 2006, Felsberg et al. 2004). It is detected in approximately $70 \%$ of oligodendrogliomas and $50 \%$ of oligoastrocytomas (Felsberg et al. 2004) and is associated with longer overall survival (Cairncross et al. 2006, van den Bent et al. 2006, Felsberg et al. 2004). The prognostic role of this co-deletion in malignant gliomas has been investigated in several studies. Isolated deletions of $1 \mathrm{p}$ or $19 \mathrm{q}$ in glioblastoma multiforme were detected in 
$6.2 \%$ and $5.3 \%$, respectively (Kaneshiro et al. 2009). However, no study could show a significant prognostic impact on overall survival of patients suffering from malignant glioma (Boots-Sprenger et al. 2013, Kaneshiro et al. 2009, Pinto et al. 2008, Brat et al. 2004, Smith et al. 2000).

NogoA (neurite outgrowth inhibitor A) is a member of the reticulon gene family, a group of proteins that are associated with the endoplasmic reticulum (GranPre et al. 2000). The name derives from the first discovered role of this protein in the inhibition of neuroregeneration (Fournier et al. 2001, Chen et al. 2000). The encoding gene produces 3 different proteins, Nogo-A, -B and -C (Chen et al. 2000). There is evidence that NogoA may also play a role in differentiating oligodendrogliomas from diffuse and anaplastic astrocytomas (Marucci et al. 2012, Kuhlmann et al. 2008). It was also discovered to be a positive prognostic marker, whose expression negatively correlated with malignancy in oligodendrogliomas (Xiong et al. 2007). Two recent studies on this matter also investigated the expression of NogoA in several other tumors, including glioblastoma. Interestingly in both publications, a subgroup of approximately $20 \%$ of GBMs showed highly increased expression levels of this protein. However, only small groups of 30 and 29 GBMs were evaluated by Marucci et al. and Kuhlmann et al. , respectively (Marucci et al. 2012, Kuhlmann et al. 2008). There has not yet been a study that delineates the prognostic role of NogoA expression and its possible correlation with clinical data in a large group of GBMs.

The oligodendrozyte transcription factor 2 (OLIG2) plays a major role in the structural development of the spinal cord. It promotes the differentiation of motor neurons and oligodendrocytes (Zhou and Anderson 2002) and is a member of the basic helix-loop-helix protein (bHLH) family (Takebayashi et al. 2000, Zhou et al. 2000). Despite its crucial function for CNS development, OLIG2 is also linked to brain tumor development. Increased expression levels can be found in diffuse gliomas including astrocytomas (Marie et al. 2001, Ligon et al. 2004). Thus it is not restricted to tumors of oligodendroglial origin. Several studies suggest OLIG2 to be a key factor responsible for proliferation of glioma cells. As an example, it was shown that OLIG2 directly inhibits the tumor suppression factor p21 in neural progenitor and glioma cells (Ligon et al. 2007). It also hinders the function of p53, regarding its response towards genetic damages (Mehta et al. 2011). The proliferative impact of OLIG2 seems to be associated with the phosphorylation of a triple serine motif (Sun et al. 2011).

In GBM the role of OLIG2 is somewhat unclear. Compared to lower graded anaplastic oligodendrogliomas and astrocytomas, expression levels of OLIG2 in GBMs are decreased (Onishi et al. 2003). Recently Fu et al. investigated the impact of the novel HSP990 inhibitor NVP-HSP990 in GBM cell lines. HSP990 is a molecular chaperone, which is involved in many signaling pathways that are believed to go astray 
in gliomagenesis. The obtained results suggest that higher expression rates of OLIG2 in GBM cells are associated with increased sensitivity towards NVP-HSP990 (Fu et al. 2013, Menezes et al. 2012, Whitesell and Lindquist 2004, Picard 2002). A recent publication showed that OLIG2 serves as a marker for glioma stem cells (Trepant et al. 2014). No data has yet been published on the prognostic and clinical role of OLIG2 expression levels in glioblastoma.

\subsubsection{Ki67}

Ki67 is a nuclear protein that is associated with cell proliferation. The name originates from the german city of Kiel where the first monoclonal antibody was developed (Gerdes et al. 1983, Scholzen and Gerdes 2000). Nowadays, it is a widespread tool in pathology laboratories to evaluate the proliferation index of tumor cells. Ki67 immunopositivity is cell cycle dependent and absent in resting cells (Schlüter et al. 1993, Braun et al. 1988). It is linked to rRNA synthesis and its inactivation leads to inhibition of ribosomal RNA production (Bullwinkel et al. 2006, Rahmanzadeh et al. 2007). A correlation between Ki67 expression and WHO astrocytoma grade is well established (Karamitopoulou et al. 1994, Khalid et al. 1997, Di et al. 1997, Heesters et al. 1999, Johannessen and Torp 2006). On the other hand, the prognostic value of Ki67 expression levels among astrocytomas and especially glioblastomas is still unclear (Johannessen and Torp 2006). There are several studies that suggest a significant disadvantage regarding the patients' clinical course if higher expression levels are detected in astrocytoma tissue (Neder et al 2004, Di et al. 1997, Sallinen et al. 1994, Jin et al. 2011). On the contrary a number of authors state that Ki67 does not represent a prognostic marker (Vaquero et al. 2000, Litofsky et al. 1998, Chiba et al.2010, Berghoff et al. 2013). Studies that specifically addressed the prognostic value of Ki67 expression in glioblastomas are rare and also produced contradictory results. A series of 38 and 37 GBMs investigated by Vaquero and Chiba, respectively, did not show Ki67 to have any prognostic impact (Chiba et al. 2010, Vaquero et al. 2000). In 2011 a larger study conducted by Jin et al. involved 156 glioblastomas. The cutoff was set at an expression rate of $25 \%$ of tumor cells. Individuals with increased expression rates $(62.8 \%$ of the samples) showed significantly shorter progression free and overall survival, while there was no difference in gender, age, extent of resection and KPS. Thus Jin et al. proclaimed Ki67 to be an independent prognostic marker (Jin et al. 2011). 


\subsubsection{P53}

One of the most important cancer genes is TP53. It encodes the tumor suppressor protein p53 and plays a major role in the inhibition of cell proliferation. When a cell encounters a stress signal or acquires DNA-damage p53 can block the cell cycle and even induce programmed cell death (apoptosis). In this way the protein can single out potential cancerous cells and thus suppress tumor development (Vousden and Prives 2009). Due to its genome stabilizing function it was awarded the role of the "guardian of the genome" (Lane 1992). Following the loss of p53 function, a cell can uncontrollably acquire numerous additional mutations that finally drive cancer development (Yahanda et al. 1995). Mutations of TP53 are frequently found in many human cancers, including brain tumors (Hollstein et al. 1991). In vitro, astrocytes of TP53 knock-out mice show a higher proliferation rate compared to their wildtype counterparts (Bögler et al. 1995). The addition of the missing TP53 via transduction into p53-deficient glioblastoma cells concluded in increased rates of apoptosis (Gomez-Manzano et al.1997). Mutations of the TP53 signaling pathway belong to the common genetic alterations of glioblastoma multiforme. The Cancer Genome Atlas Research Network revealed that $87 \%$ of primary glioblastomas show alterations of genes involved in the TP53 signaling pathway. Mutations of TP53 itself occurred in approximately 35\% (TCGA 2008). As mentioned above, 4 subgroups of GBM were developed depending on multivariate analysis of genetic alterations. The so-called proneural subtype included most cases with TP53 mutations. It showed significant association with younger age, secondary GBMs and a non-significant trend towards longer survival (Verhaak et al. 2010). The higher frequency of TP53 mutations in secondary GBMs (63\% compared to $28 \%$ ) had already been described earlier by Ohgaki and Kleihues. TP53 mutations are already present in the lower-grade precursor lesions of secondary GBMs (Ohgaki and Kleihues 2009, Ohgaki and Kleihues 2004).

There were several other studies in the past delineating the prognostic role of TP53 mutations in GBM. Most studies suggest no significant prognostic impact of TP53 mutations (Felsberg et al. 2009). In 2004 Ohgaki et al. assessed a large patient collective of 386 glioblastomas and found TP53 mutations to be of prognostic significance. Patients with mutated tumors seemed to survive longer. However, these patients were younger and after age-adjusted multivariate analysis the difference lost statistical significance (Ohgaki et al. 2004). Similar results were attained by other research groups (Batchelor et al. 2004, Smith et al. 2001) suggesting that TP53 mutations in GBM are rather associated with younger age and better clinical status at the time of diagnosis. Even though this issue seems to have been resolved, data has not yet been published regarding differing mutation rates of TP53 and a possible association with overall survival and other clinical data. 


\subsubsection{GFAP}

Glial fibrillary acidic protein (GFAP) is a type III intermediate filament protein which is generally expressed in astrocytes and ependymal cells of the brain (Eng et al. 1971 and 2000, Roessmann et al. 1980). The protein plays an important role in brain development and the recovery of the central nervous system from conditions such as trauma or inflammation which lead to an increase in GFAP expression in astrocytes (Smith and Eng 1987, Middeldorp and Hol 2011). Astrocytic tumors are also known to hold a high expression rate of this filament (Abaza et al. 1998, Hamaya et al. 1985, Jung et al. 2007). In vitro studies suggested a correlation of loss of GFAP expression and dedifferentiation of astrocytoma cells (Wilhelmsson et al. 2003, Chen and Liem 1994, Rutka et al. 1994, Weinstein et al. 1991). Several attempts were made to show a correlation between GFAP expression levels and clinical course of astrocytomas. In 1978 Jacque et al. stated that low levels of GFAP in glial tumor tissue were associated with malignant grading while higher levels were rather found in healthy astrocytic tissue (Jacque et al 1978). In contrast, Reyaz et al. constituted a significant correlation of GFAP immunoreactivity with WHO grading of astrocytic brain tumors (Reyaz et al. 2005) while Heo et al. published similar results for astrocytic tumors of the spinal cord (Heo et al. 2012). Interestingly, in cases of leptomeningeal spread of intracranial GBMs the disseminated cells showed lower GFAP expression levels than intramedullary or intracranial lesions, suggesting a tendency to disseminate due to loss of astrocytic differentiation (Maslehaty et al. 2011). GFAP was also investigated regarding its use as a tumor marker. Serum measurements of GFAP from patients suffering from high grade gliomas showed some interesting results. Elevated preoperative GFAP serum levels correlated with tumor volume and WHO grading while it presented as a highly specific tumor marker (Jung et al. 2007, Brommeland et al. 2007, Wei et al. 2013). The question whether there is a correlation between GFAP expression and prognosis or clinical features within glioblastoma has been addressed by Schmidt et al. in 2002 without any significant finding when assessing 97 GBMs (Schmidt et al. 2002). 


\subsection{Aims of this Dissertation}

As delineated above there are several immunohistochemical markers whose prognostic value in glioblastoma is still unclear. The aim of this dissertation is to evaluate the prognostic significance of several tumor markers and their possible correlation with clinical parameters. With a sufficiently sized patient collective of 120 GBMs new insights can be attained regarding the prognostic role of expression levels of NogoA and OLIG2, as markers of oligodendroglial differentiation, as well as GFAP and Ki67. Mutations of p53 and IDH-1 will also be studied. In the long-run we hope that our findings will contribute to the development of targeted individual therapies for glioblastomas. 


\section{Chapter 2}

\section{Materials and Methods}

\subsection{Patient Collective}

This study included patients that were diagnosed with glioblastoma multiforme between 10th March 1997 and 10th May 2011 at the University Medical Center Göttingen. All but three patients had already succumbed to their disease by the end of this study. All considered cases were pseudonymized and the associated clinical information was organized in a brain tumor database (PD Dr. Florian Stockhammer, Department of Neurosurgery, University Medical Center Göttingen). Clinical information was obtained from medical reports and from the clinical cancer registry. The patient selection was done in cooperation with the Department of Hematology and Oncology of the University Medical Center Göttingen (PD Dr. Tobias Pukrop). A permission to use archival material for immunohistochemistry of tumor cell differentiation markers and clinical data was obtained from the Ethics Committee of the University Medical Center Göttingen (Antrag-Nr. 24/10/05, Amendment 21/3/11 and Antrag/Nr. 03/10/14).

Considered were cases that received adjuvant chemotherapy with an alkylating agent and external beam radiation therapy. From a total of 120 patients, 97 were treated with temozolomide alone while 8 were given carmustine (BCNU) additionally, which was administered locally in form of a Gliadel ${ }^{\circledR}$ wafer. 15 patients received nimustine (ACNU) as a single chemotherapeutic agent. All 120 patients were treated with adjuvant radiotherapy including up to 2 centimeters beyond the tumor margin. 93 tumors were extensively resected (gross total resection) while 26 were only partially removed. In one case the patient merely underwent a biopsy to confirm the diagnosis of glioblastoma multiforme and did not receive further tumor resection. 
Cases were excluded if the paraffin-embedded tumor sample was not suitable for tissue microarray processing or if the tissue sample was not available. Patients were also excluded if only incomplete data on their clinical course and management could be obtained.

\subsection{Biospecimen Selection}

Paraffin-embedded tumor samples were acquired from the tissue bank of the Department of Neuropathology of the University Medical Center Göttingen. The samples were received from diagnostic biopsies and from therapeutic surgical tumor resections that were performed in the Department of Neurosurgery of the University Medical Center Göttingen between 10th March 1997 and 10th May 2011. All cases received adjuvant chemotherapy with alkylating agents and external beam radiation therapy. Only samples that led to the initial diagnosis were used for this study. The diagnoses were made histopathologically according to the WHO classification of astrocytic tumors (Louis et al. 2007). In most cases there were several paraffin-embedded tissue samples for one diagnostic event. Microscopic evaluation of the respective hematoxylin and eosin sections (HE-sections) was performed to single out the eligible samples. The focus was on samples that presented the diagnostic criteria of the tumor and an area of viable tumor tissue that was large enough to allow further processing into tissue microarrays (TMAs). Some paraffin-embedded tissue samples showed poor suitability for TMAs. The paraffin blocks held tissue samples that were too small to be punched out with a $2 \mathrm{~mm}$-bore biopsy puncher (see Chapter 2.2) or samples that mainly consisted of necrotic areas or blood (Figure 2.1). These samples were not included into the study since they could not undergo tissue microarray processing.

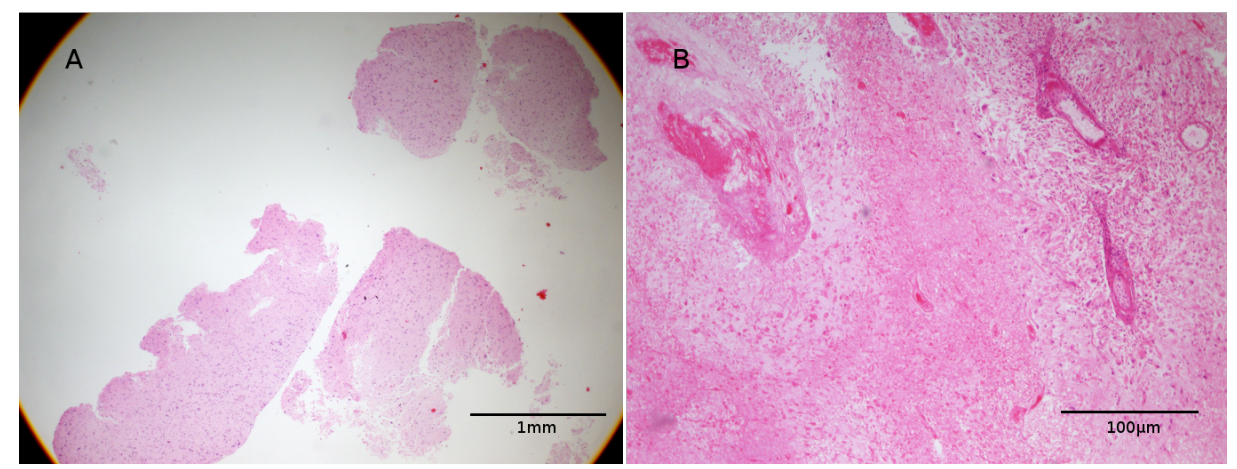

Figure 2.1: Microcopic exclusion criteria. A: HE-section of a paraffin-embedded tissue sample that was too small for a $2 \mathrm{~mm}$ biopsy punch (20-fold magnification). B: HE-section of a paraffin-embedded tissue sample that mainly consisted of necrotic areas with too little viable tumor tissue for TMA (40-fold magnification). 
HE sections of all tumor samples that were selected for further work up, were sent to Professor Christian Hartmann, director of the Department of Neuropathology at the Hannover Medical School. There the histopathology of each tumor sample was reviewed and areas best suitable for tissue microarrays were marked. The marked HE sections were sent back to the University Medical Center Göttingen and the paraffinembedded tumor samples underwent further processing into tissue microarrays. Figure 2.2 shows a typical area on a HE-section that was suitable for TMA processing.



Figure 2.2: Area suitable for TMA. HE-section of a paraffin-embedded tissue sample (A: 20-fold magnification, B: 100-fold magnification). The tissue shows a high density of viable tumor cells and is large enough to be punched out with a $2 \mathrm{~mm}$-bore biopsy puncher.

\subsection{Tissue Microarray (TMA)}

To enable comparative analysis of several tissue samples on one microscopic slide, tissue microarrays (TMA) were utilized. With this technique a large number of tissue samples were arranged on a single paraffin block, which made further processing more comparable due to uniformity of immunhistochemical staining conditions. In this way a large number of samples underwent comparative evaluation.

\subsubsection{Arrangement of Samples and Controls}

Each recipient block held 60 slots each with a $2 \mathrm{~mm}$ diameter, 10 slots a row. One row of each recipient block was filled with 6 controls and the 4 remaining slots were left empty. The row number of the control-row indicated the numbering of the TMA. Each TMA block held a unique positioning of its control-row to minimize the risk of accidental mixing up of TMA slides. Two of the 6 control samples comprised of normal cerebellum and normal hippocampus and served as healthy tissue controls. The remaining 4 control samples were chosen according to the aim of the study. Oligodendrogliomas WHO grade II and III served as OLIG2 and NogoA positive controls while a sample of a pilocytic astrocytoma WHO grade I was used as a positive control for GFAP. A cerebral melanoma metastasis served as a non-glial tumor control. 


\subsubsection{TMA Procedure}

Representative regions of paraffin-embedded tumor samples were punched out with a 2mm-bore biopsy puncher and placed into the recipient paraffin block (see Figure 2.3). In most cases there was enough tumor tissue available to take two samples from each case. Prior to this step the sample blocks and the recipient blocks were warmed up at $37^{\circ} \mathrm{C}$ for 2 hours to prevent cracking of the sample blocks or breaking of the sample cylinder due to brittle paraffin. To ensure an even surface for proper microtomy, each sample was gently pushed into the slot with a flat object until it formed a level surface with the recipient block. After all slots of the block had been filled with tumor samples and control tissue, it was placed in a fitting basemold, facedown, and heated at $60^{\circ} \mathrm{C}$ until the paraffin block appeared completely transparent. Subsequently a tissue-embedding cassette was placed on top of the block and the basemold was filled with warm liquid paraffin until it was covered up through the grit of the cassette. Then the basemold holding the TMA block was placed on a cold surface $\left(-10^{\circ} \mathrm{C}\right)$ for approximately 10 minutes until the paraffin became solid and the new block could easily be removed from the basemold. Surplus paraffin at the edges of the cassette of the new block was cut off with a knife to ensure proper placement into the microtome (see Figure 2.4).
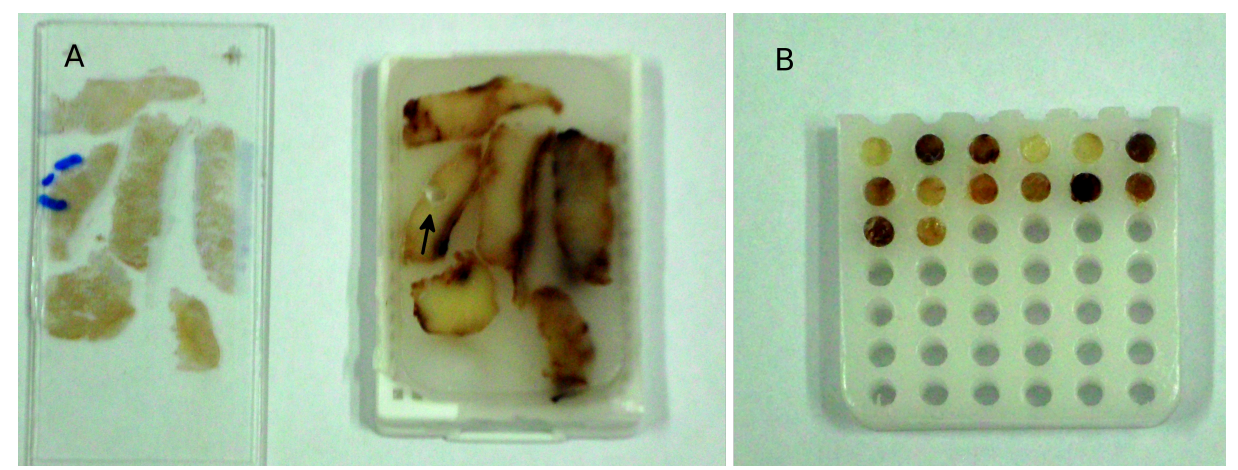

Figure 2.3: Illustration of the TMA procedure. A: Marked representative region on a stained slide and the corresponding paraffin-embedded tissue block with a sample already punched out (arrow). B: Recipient block filled with several punched out samples cylinders. 


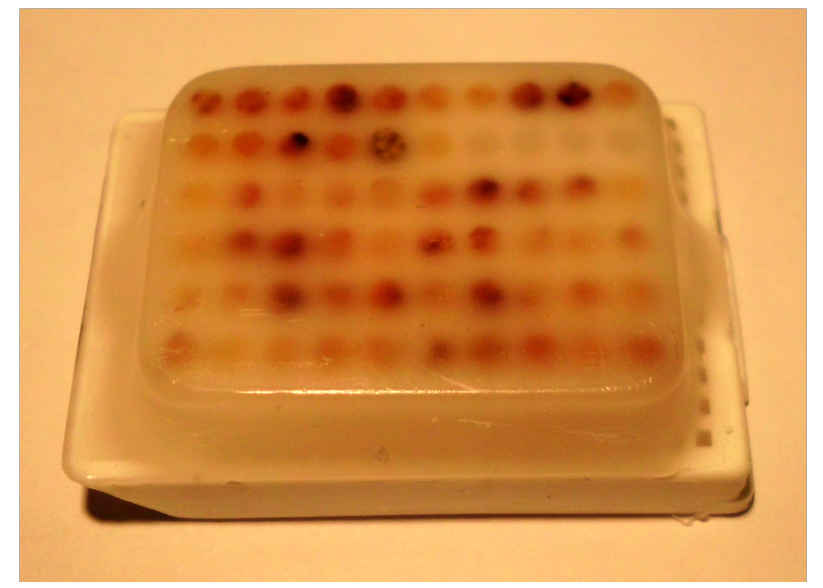

Figure 2.4: Completed TMA-block right before microtomy.

Table 2.1: Materials used for Tissue Microarray and microtomy.

\begin{tabular}{lc}
\hline Material & Provider $^{1}$ \\
\hline Adhesion microscope slides & Menzel Gläser $^{1}$ \\
Basemold for tissue embedding & Thermo Fisher Scientific $^{2}$ \\
Cooling surface & MEDITE $^{3}$ \\
Disposable Biopsy Punch $2 \mathrm{~mm}$ & pfm medical $^{4}$ \\
Incubator & Memmert $^{5}$ \\
Microscope & Olympus $^{6}$ \\
Microtome & Leica $^{7}$ \\
Microtome blades & Leica $^{7}$ \\
Paraffin & McCormick Scientific $^{8}$ \\
Paraffin embedding machine & MEDITE $^{3}$ \\
Quick-Ray & Recipient Block $2 \mathrm{~mm}$ \\
Tissue embedding cassettte & Sakura $^{\circledR 8}$ \\
\hline
\end{tabular}

\footnotetext{
${ }^{1}$ Menzel Gläser GmbH, Braunschweig, Germany

${ }^{2}$ Thermo Fisher Scientific Inc., Waltham, MA, USA

${ }^{3}$ MEDITE GmbH, Burgdorf, Germany

${ }^{4}$ pfm medical ag, Köln, Germany

${ }^{5}$ Memmert GbmH + Co.KG, Schwabach, Germany

${ }^{6}$ Olympus Deutschland GmbH, Hamburg, Germany

${ }^{7}$ Leica Microsystems GmbH, Wetzlar, Germany

${ }^{8}$ VWR International GmbH, Darmstadt, Germany

${ }^{9}$ Sakura Finetek Europe B.V, Alphen aan den Rijn, The Netherlands

${ }^{10} \mathrm{KABE}$ LABORTECHNIK GmbH, Nümbrecht Elsenroth, Germany
} 


\subsection{Microtomy and Paraffin Section Preparation}

Before processing into tissue microarrays, each paraffin-embedded tumor sample underwent microtomy to produce $2 \mathrm{HE}$ sections. One of the HE sections was categorized for an overview of all included samples. The other HE section was sent to the Neuropathology Department of the Hannover Medical School as described above (see chapter 2.2.1). The microtome was also used for the preparation of paraffin sections from the tissue microarray blocks, which then underwent immunohistochemical staining. Before placement into the microtome, each sample was placed facedown on a $12^{\circ} \mathrm{C}$ cooling plate to make the paraffin wax harder. This ensured a better support for the tissue sample within and allowed for thinner cutting. Subsequently, the cassettes of TMA-blocks were adequately fixed in the object clamp and positioned to meet the cutting edge of the knife with their complete surface. The angle of the blade holder was set at 3 degrees. Trimming of the TMA-blocks was carefully performed at a thickness of $10 \mu \mathrm{m}$ until the first sample cylinders were obtainable. From then on paraffin sections were cut at $3 \mu \mathrm{m}$. To flatten out and expand, paraffin sections were then placed on a water surface at a temperature of $58^{\circ} \mathrm{C}$ for a few seconds and subsequently placed on a slide. The water was skimmed frequently between microtomy of different blocks to avoid cross-contamination. All sections were dried in an upright position at room temperature overnight.

\subsection{HE Staining}

Hematoxylin and Eosin (HE) staining enabled the histopathological diagnosis of the sample as well as the assessment of suitability for tissue microarrays. For these purposes paraffin sections of each sample were placed into an incubator at $58^{\circ} \mathrm{C}$ for 10 minutes and subsequently deparaffinized by bathing in xylene 3 times for 4 minutes and once in iso-xylene for 4 minutes. The sections were then hydrated in $100 \%$ alcohol two times for 3 minutes each and in a serial of diluted alcohol three times (90\%, 70\% and $50 \%$ ) for 2 minutes each. To finish the hydration process, the sections were placed in distilled water for 2 minutes. The first staining step in Mayer's hematoxylin was done for 8 minutes. Subsequently the sections were briefly dipped in distilled water and $\mathrm{HCl}$-alcohol. To remove all residues the samples were then carefully rinsed under running tap water from the reverse side for 10 minutes. For the second coloration step the sections were put into an Eosin bath for 5 minutes, followed by the placement into distilled water for 2 minutes. After completion of the two coloring processes, all sections underwent dehydration by bathing in a serial of diluted alcohol three times (50\%, 70\% and 90\%) for 2 minutes each. The samples were then placed into $100 \%$ alcohol twice for 3 minutes each. Then they were set into an iso-xylene bath for 4 
minutes and subsequently into a xylene bath three times for 4 minutes each. Finally all stained sections were mounted with DePex and dried at room temperature before undergoing microscopy.

Table 2.2: Materials used for $\mathrm{HE}$ staining.

\begin{tabular}{lc}
\hline Material & Provider \\
\hline DePex & SERVA $^{1}$ \\
Eosin G Certistain & Merck $^{2}$ \\
Hydrogen chloride $(\mathrm{HCl})$ & Merck $^{2}$ \\
Incubator & Memmert $^{3}$ \\
Isopropyl alcohol & Chemie Vertrieb Hannover $^{4}$ \\
Mayer's hematoxylin & Merck $^{2}$ \\
Xylene & J.T.Baker $^{5}$ \\
\hline
\end{tabular}

\subsection{Immunohistochemistry}

Immunohistochemistry is a frequently used method to detect antigens on tissue samples with the help of specifically binding antibodies. In this study indirect immunohistochemistry was used to allow comparative analysis of the expression rates of GFAP, Ki67, P53, OLIG2 and NogoA as well as the detection of IDH-1(R132H)-mutations among the tumor samples. The method of immunohistochemical staining is depicted in Figure 2.5. The primary antibody is directed at the protein of interest and binds to it with its Fab (Fragment, antigen binding) region. Depending on the origin of the primary antibody (mouse, rabbit, goat, etc.), the secondary antibody is selected accordingly to allow binding to the Fc (Fragment, crystallizable) region of the primary antibody. Several secondary antibodies can bind to the primary antibody, which causes significant signal amplification. The secondary antibodies carry several biotin molecules on their Fc region. Due to its high affinity to avidin-bound enzymes, biotin can recruit avidin-peroxidase complexes to the antigen of interest. Subsequently $\mathrm{H}_{2} \mathrm{O}_{2}$, the substrate of the peroxidase, and 3,3'-diaminobenzidine (DAB), an electron donator, are added to the tissue sample. This leads to the oxidation and precipitation of DAB restricted to the areas where the primary and secondary antibodies have successfully bound to the antigen of interest. The precipitation of oxidized DAB results in a brown discoloration of the tissue.

\footnotetext{
${ }^{1}$ SERVA Electrophoresis GmbH, Heidelberg, Germany

${ }^{2}$ Merck KGaA, Darmstadt, Germany

${ }^{3}$ Memmert GmbH + Co.KG, Schwabach, Germany

${ }^{4} \mathrm{CVH}$ Chemie-Vertrieb GmbH \& Co. Hannover KG, Hannover, Germany

${ }^{5}$ Avantor Performance Materials, Center Valley, PA, USA
} 


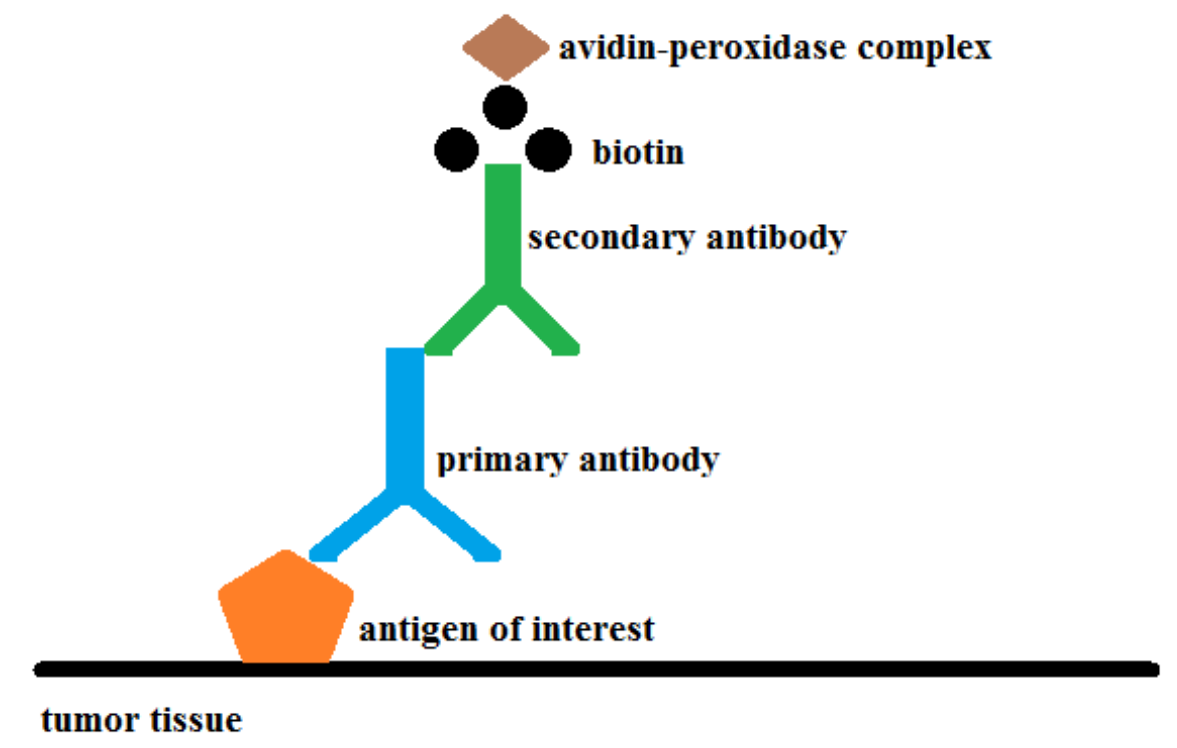

Figure 2.5: Indirect IHC and the avidin-biotin complex.

The first step was heating up the dried sections at $58^{\circ} \mathrm{C}$ for at least 10 minutes in an incubator to melt the paraffin. Subsequently the samples were bathed in xylene 4 times for 5 minutes each. After a quick wash in isoxylene for 1 minute the sections were further deparaffinized in 100\% alcohol twice for 4 minutes each and afterwards consecutively in 90\%, 70\% and 50\% alcohol for 3 minutes each. The deparaffinization process was finished with a thorough wash in distilled water. In order to break down molecular cross links formed by formalin fixation, the samples were treated with antigen retrieval reagents, depending on the applied primary antibody. For immunohistochemsitry with P53, Ki67 or NogoA the samples were treated with 10mM citrate, for OLIG2 tris/borate/EDTA buffer (TBE) was utilized. GFAP staining did not require such pretreatment. For the purpose of antigen retrieval all samples were transferred into a plastic sample holder, which was filled with the desired antigen retrieval reagent and underwent 3 heating cycles in a microwave for 3-10 minutes, depending on the amount of sample holders placed in the microwave. After each heating cycle evaporated reagent was replaced to ensure that the tissue samples were completely covered throughout the antigen retrieval process. Subsequently the hot citrate/TBE was washed out of the sample holder with room temperature distilled water and the samples were transferred into a glass sample holder filled with phosphate buffered saline (PBS). In the next step the samples were treated with $3 \%$ hydrogen peroxide for 10-15 minutes to block endogenous peroxidases. Since the antigen of interest was later made visible with the help of peroxidase, endogenous enzymes of this type would cause false positive results. The samples were then washed in distilled water and subsequently in PBS. At this point samples that underwent staining for NogoA or OLIG2 received an additional treatment. After a thorough wash in PBS the slides were placed 
into an upright position to allow the PBS to drain off the tissue samples. Remaining PBS was carefully wiped off with a tissue without touching the tumor samples. Subsequently, $100 \mu \mathrm{L}$ of Triton (for NogoA staining) or $0.2 \%$ Casein (for OLIG2 staining) was applied onto the tumor tissue samples. Cover slips were placed on top to ensure even distribution of the applied fluid. The slides were placed into a humidity chamber for 1 hour (NogoA) or 15 minutes (OLIG2). Afterwards the samples were thoroughly washed in PBS 3-4 times and entered the blocking step. Before the primary antibody was applied to the slides, each tissue sample was covered in $10 \%$ fetal calf serum (FCS) diluted in PBS to block unspecific proteins. Depending on the size of the tissue sample on the slide, 100-200 $\mu \mathrm{L}$ FCS was applied for approximately 30 minutes. The slides were placed into a humidity chamber at room temperature. For immunohistochemical staining for OLIG2, the blocking process, as well as all other steps, were done with normal goat serum (NGS) instead of FCS. This was due to the origin of the secondary antibody. Afterwards the slides were put into an upright position to allow the blocking solution to drain off the tumor tissue. Remaining $10 \%$ FCS could dilute the primary antibody solution and was therefore carefully soaked up with tissue without touching the tumor samples. The primary antibody was diluted in $10 \%$ FCS and $20-100 \mu \mathrm{L}$ was applied to each slide, depending on the size of the tumor tissue. Cover slips were placed on top to ensure even distribution of the antibody. The slides incubated in a humidity chamber at $4^{\circ} \mathrm{C}$ overnight. Before the secondary antibody was applied all slides were stripped off their cover slips and washed thoroughly in PBS for at least 4 times. Remaining PBS was drained by putting the slides in an upright position and carefully soaking up excess PBS with tissue without touching the tumor samples. The secondary antibody was chosen according to the origin of the primary antibody (anti-mouse or anti-rabbit). It was also diluted in $10 \%$ FCS. $100 \mu \mathrm{L}$ were applied to each tumor tissue slide for $1 \mathrm{~h}$ in a humidity chamber at room temperature without a cover slip. Subsequently all slides were thoroughly washed in PBS for at least 4 times and afterwards treated with avidin-peroxidase, at a dilution of 1:1000 in PBS. $100 \mu \mathrm{L}$ were applied to each sample with a cover slip on top to ensure even distribution. The samples incubated in a humidity chamber at room temperature for 45 minutes. This was followed by another thorough washing process in PBS (4 times). For the developing process 3,3-diaminobenzidine (DAB) was used as a chromogen. 25 mg of DAB and $20 \mu \mathrm{L}$ of $30 \%$ hydrogen peroxide were diluted in $50 \mathrm{ml}$ of PBS in a glass sample holder. The samples were placed into the developing solution and were viewed under the microscope every couple of minutes to avoid overstaining and background activity. When satisfactory staining was accomplished the developing process was stopped with a thorough wash under running distilled water. For counterstaining of the nuclei all samples we treated with Mayers Hematoxylin for 20 seconds (5 seconds maximum for OLIG2 immunohistochemistry) and briefly washed in distilled 
water. Afterwards they were differentiated by a quick wash in $1 \%$ hydrochloric acid alcohol and thoroughly washed under running tap water for 10 minutes. Dehydration of the samples was achieved by repeating the steps of the deparaffinization process in a reverse order. Starting with a quick wash in distilled water, the slides consecutively went through a bath in 50\%,70\%, 90\% and 100\% alcohol for 3 minutes each. After a quick wash in isoxylene the samples were dehydrated in xylene four times for 5 minutes each. And finally the stained slides were mounted with DePex and dried at room temperature. 
Identification of Prognostically Relevant Cellular Markers of Differentiation in Glioblastoma

Table 2.3: Materials used for immunohistochemistry.

\begin{tabular}{|c|c|}
\hline Material & Provider \\
\hline Casein & Merck $^{1}$ \\
\hline Citric acid monohydrate & Merck $^{1}$ \\
\hline DAB & Sigma Aldrich ${ }^{2}$ \\
\hline DePex & Serva $^{3}$ \\
\hline Disodium tetraborate & Carl Roth ${ }^{4}$ \\
\hline Ethylenediaminetetraacetic acid (EDTA) & Carl Roth ${ }^{4}$ \\
\hline ExtrAvidin Peroxidase & Sigma Aldrich ${ }^{2}$ \\
\hline Fetal calf serum (FCS) & Biochrom $^{5}$ \\
\hline Humidity chamber & in-house manufacture \\
\hline Hydrogen chloride (HCL) & Merck $^{1}$ \\
\hline Hydrogen peroxide $30 \%$ & Merck $^{1}$ \\
\hline Incubator & Memmert $^{6}$ \\
\hline Isopropyl alcohol & Chemie Vertrieb Hannover ${ }^{7}$ \\
\hline Mayer's hematoxylin & Merck $^{1}$ \\
\hline Microwave & Panasonic $^{8}$ \\
\hline Microscope & Olympus $^{9}$ \\
\hline Microscope camera & Olympus ${ }^{9}$ \\
\hline Microscope cover slips & Menzel Gläser ${ }^{10}$ \\
\hline Normal goat serum (NGS) & GE Healthcare ${ }^{11}$ \\
\hline Phosphate buffered saline (PBS) & AppliChem ${ }^{12}$ \\
\hline Refrigerator & Liebherr $^{13}$ \\
\hline Tris(hydroxymethyl)aminomethane & Carl $\operatorname{Roth}^{4}$ \\
\hline Triton X-100 & Merck $^{1}$ \\
\hline Xylene & GE Healthcare ${ }^{11}$ \\
\hline
\end{tabular}

\footnotetext{
${ }^{1}$ Merck KGaA, Darmstadt, Germany

${ }^{2}$ Sigma-Aldrich Co. LLC., St. Louis, MO, USA

${ }^{3}$ SERVA Electrophoresis GmbH, Heidelberg, Germany

${ }^{4}$ Carl Roth GmbH + Co.KG, Karlsruhe, Germany

${ }^{5}$ Biochrom AG, Berlin, Germany

${ }^{6}$ Memmert GbmH + Co.KG, Schwabach, Germany

${ }^{7} \mathrm{CVH}$ Chemie-Vertrieb GmbH \& Co. Hannover KG, Hannover, Germany

${ }^{8}$ Panasonic Marketing Europe GmbH, Wiesbaden, Germany

${ }^{9}$ Olympus Deutschland GmbH, Hamburg, Germany

${ }^{10}$ Menzel Gläser GmbH, Braunschweig, Germany

${ }^{11}$ GE Healthcare Europe GmbH, Freiburg, Germany

${ }^{12}$ AppliChem GmbH, Darmstadt, Germany

${ }^{13}$ Liebherr-International Deutschland GmbH, Biberach an der Riss, Germany
} 
Table 2.4: Antibodies used for immunohistochemical staining.

\begin{tabular}{llll}
\hline & Provider & Isotype & Dilution \\
\hline Primary & & & \\
GFAP & Dako $^{1}$ & rabbit & $1: 1000$ \\
Ki67 & Dako $^{1}$ & mouse & $1: 500$ \\
NogoA & Santa Cruz $^{2}$ & mouse & $1: 500$ \\
OLIG2 & IBL $^{3}$ & rabbit & $1: 300$ \\
P53 & BD Bioscience $^{4}$ & mouse & $1: 25$ \\
\hline Secondary & & & \\
Anti-rabbit & GE Healthcare & & $1: 200$ \\
Anti-mouse & GE Healthcare & & $1: 200$ \\
Anti-mouse & Dianova $^{6}$ & & $1: 500$ \\
\hline
\end{tabular}

\subsection{Microscopic Evaluation}

The evaluation of HE stains and immunohistochemical stains was done by light microscopy under 40-, 100-, 200- and 400-fold magnification. Microscopic images were taken with an Olympus DP71 digital camera that was attached to the Olympus BX41 light microscope. The processing of digital images was done with CellSens imaging software by Olympus. A tumor sample was not considered for further evaluation if less than one third of the cylinders' diameter contained properly stained viable tumor cells. Reasons for this measure were incomplete adhesion of the sample, extensive necrosis within the sample or missing tumor tissue cylinder in the slot of the receiver block at this specific sectioning level.

\subsection{Quantification of Expression Levels}

The expression rates of antigens of interest varied throughout the tumor samples. For each tumor marker that was investigated in this study (IDH1, GFAP, Ki67, P53, NogoA and OLIG2) quantification of the expression rate was necessary for proper statistical analysis. The expression pattern of Ki67, P53 and OLIG2 is nuclear and therefore immunohistochemical staining leads to simple nuclear coloring. This allowed for counting of positive cells and estimation of the immunopositivity percentage via extrapolation throughout the sample cylinder. Since IDH1, GFAP and NogoA show

\footnotetext{
${ }^{1}$ Dako Deutschland GmbH, Hamburg, Germany

${ }^{2}$ Santa Cruz Biotechnology Inc., Heidelberg, Germany

${ }^{3}$ IBL-America, Minneapolis, MN, USA

${ }^{4}$ BD Biosciences, Heidelberg, Germany

${ }^{5}$ GE Healthcare Europe GmbH, Glattbrugg, Switzerland

${ }^{6}$ Dianova GmbH, Hamburg, Germany
} 
perinuclear staining patterns quantification was more challenging. The stained cytoplasm could often not clearly be allocated to the appropriate nuclei due to the dense structure of the tumor tissue. Therefore a scoring system was developed to allow quantification of the observed expression levels of each of the three proteins. The scoring systems are explained in detail together with the results in chapter 3.

\subsection{Statistical Analysis}

The raw data and the collected clinical information were organized with Microsoft Excel 2010. Statistical analysis was performed with the help of GraphPad Prism 5 and RStudio. Statistical significance was evaluated using the Gehan's Wilcoxon test for the comparison of Kaplan-Meier curves. The independent two-sample t-test was applied to evaluate differences in clinical status and age. Cox regression analysis was used for multivariate analysis. A significance level of p-value $<0.05$ was applied.

Table 2.5: Computer Software used in this study.

\begin{tabular}{lc}
\hline Software & Provider \\
\hline cellSens digital imaging software & Olympus $^{1}$ \\
Microsoft Office 2010 & Microsoft $^{2}$ \\
MiKTeX & Christian Schenk \\
GraphPad Prism 5 & GraphPad Software Inc. $^{3}$ \\
RStudio & RStudio Inc. \\
Texmaker & Pascal Brachet \\
\hline
\end{tabular}

\footnotetext{
${ }^{1}$ Olympus Deutschland GmbH, Hamburg, Germany

${ }^{2}$ Microsoft Corporation, Redmond, WA, USA

${ }^{3}$ GraphPad Software Inc., La Jolla, CA, USA

${ }^{4}$ RStudio Inc., Boston, MA, USA
} 
Identification of Prognostically Relevant Cellular Markers of Differentiation in Glioblastoma 


\section{Chapter 3}

\section{Results}

\subsection{Patient Cohort Characterization}

The aim of this dissertation is to assess several tumor markers and their possible correlations with clinical parameters in glioblastoma multiforme. For this purpose 120 paraffin-embedded tumor tissue samples were studied. All patients received chemotherapy with alkylating agents and radiation therapy that included cerebral tissue 2 centimeters beyond the resection cavity. In 93 cases the tumor underwent gross total resection while in 26 cases it could only be partially removed. One glioblastoma was merely biopsied to establish the histopathological diagnosis and the patient did not receive further tumor resection.

117 patients had already succumbed to their disease by the end of the study. A survival time of 24 months was exceeded in merely 18 of 120 cases (15.0\%). Only three patients lived longer than 36 months after the time of diagnosis $(2.50 \%)$. The mean overall survival of the patient cohort was 14.54 months (see Figure 3.1). The majority of the patients suffering from GBM were older than 60 years of age $(65.83 \%)$ and had a KPS of $70 \%$ or more (mean KPS 70.83\%) at the time of diagnosis (see Figure 3.2). 



Figure 3.1: Distribution of overall survival within the patient collective (120 glioblastomas). Left panel: Overall survival of the observed patient collective illustrated as a Kaplan-Meier curve. Right panel: Number of observed patients with regard to the overall survival in 6 months intervals.
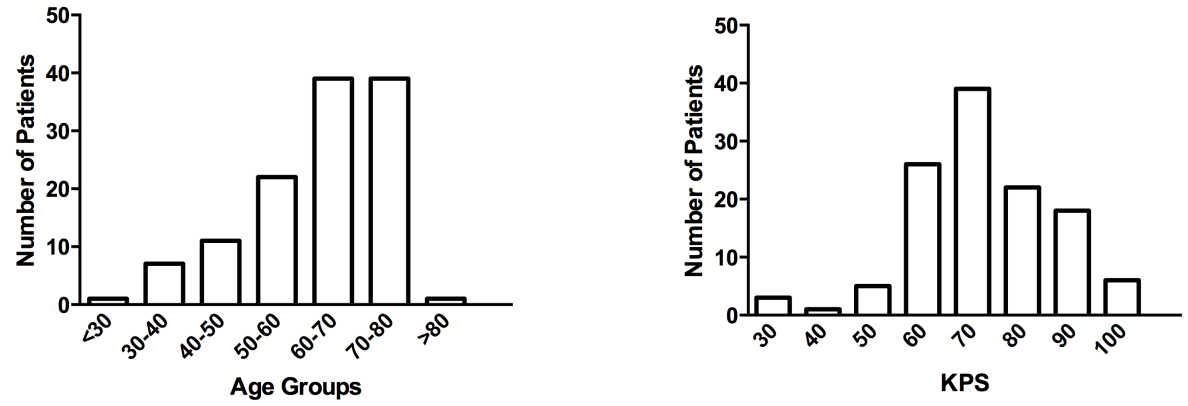

Figure 3.2: Distribution of age and KPS at the time of diagnosis of glioblastoma. 


\subsection{Prognostic Impact of Clinical Factors}

It is well established that the overall survival of patients diagnosed with GBM depends on several clinical factors such as age, clinical status and extent of surgical resection (see chapter 1.2.1). Therefore in order to identify the relative contributions of these factors to the overall survival in our patient cohort we examined age and KPS at diagnosis as well as sex, type of chemotherapy and extent of surgical resection regarding the prognostic impact on overall survival.

\subsubsection{Sex}

The data available on the prognostic impact of biological gender in GBM is contradictory with results attributing longer overall survival to both sexes. Nevertheless, a male predominance of glioblastoma is widely accepted (see chapter 1.1.1 and 1.2.1). In our patient cohort 72 of 120 patients were male and 48 female, resulting in a male to female incidence ratio of exactly 1.50 (see figure 3.1). Female patients tended to have a slightly shorter mean overall survival (13.74 months) compared to their male counterparts (15.07 months). However, no statistically significant difference was found $(\mathrm{p}=0.2456)$. Out of 10 patients with an overall survival of less than 6 months 7 were male. Eighteen patients exceeded an overall survival of 24 months of which 12 were men. The mean age of the two gender groups showed no difference (males 62.36, females 62.12, $\mathrm{p}=0.9138$ ) while males presented with a slightly higher clinical status (KPS) at the time of diagnosis without statistical significance (73.33\% compared to $70.21 \%, \mathrm{p}=0.2552$ ) (see Figure 3.1). There was no difference in the proportion of female and male patients who received gross total resection $(77.08 \%$ of female and $77.78 \%$ of male patients, respectively). 




Figure 3.3: No influence of sex on overall survival in GBM. A: The overall survival of both genders is depicted as a Kaplan-Meier curve. B: The mean overall survival according to the gender depicted as bar graphs. $\boldsymbol{C}$ and $\boldsymbol{D}$ : Age and KPS of male and female patients with no apparent difference. Bars represent mean values with standard deviation. Asterisks represent statistically significant differences (p-values $<0.05)$.

\subsubsection{Age at Diagnosis}

In glioblastoma multiforme younger age at the time of diagnosis has an established positive prognostic impact. Patients that are younger than 60 years show a longer overall survival. An age-cutoff at 60 years is an established prognostic factor which is widely used to make decisions concerning adjuvant treatment (see chapter 1.2.1).

In this patient cohort the mean age at the time of diagnosis was 62.26 years. 79 of 120 patients were over 60 years of age $(65.33 \%)$ while the youngest was 24 and the oldest 80 years old (see figure 3.2). The cohort was divided into groups according to patient age. Cutoffs were placed at 55, 60, 65 and 70 years. The corresponding Kaplan-Meier curves and bar graphs are shown in figures 3.4 and 3.5. All 4 cutoffs suggest a prognostic difference in favor of the younger patient group, yet not all reached statistical significance. The cutoff at 55 years of age at the time of diagnosis divided the patient collective into 31 under and 89 patients over 55 . The younger collective had a mean overall survival of 17.19 months compared to 13.61 months of their older counterparts. However, statistical analysis did merely indicate a trend without statistical significance $(\mathrm{p}=0.0815)$. On the other hand, the cutoffs at 60,65 and 70 years of age all showed statistical significance. Patients exceeding the age 
of 60 displayed a mean overall survival of 13.36 months while younger individuals lived 16.81 months on average $(\mathrm{p}=0.0234)$. The difference between older and younger patient groups at the cutoff at 65 years was even more apparent with 12.00 and 16.91 months, respectively $(p=0.0010)$. Even the cutoff at 70 years showed prognostic value with a mean overall survival of 12.06 and 15.77 months of patients above and below 70 years of age, respectively $(\mathrm{p}=0.0278)$. In the multivariate analysis (see section 3.4) patient age was not a significant independent prognostic factor in glioblastoma $(\mathrm{p}=0.4052$, see section 3.4$)$.


D



Figure 3.4: Younger age is a significant prognostic factor in GBM I. Kaplan-Meier curves of different age groups of patients suffering from GBM. The prognostic impact of age was evaluated at cutoffs at $55(\boldsymbol{A}), 60(\boldsymbol{B}), 65(\boldsymbol{C})$ and 70 years $(\boldsymbol{D})$. In all cutoff analyses the younger age group showed a longer mean overall survival, with all cutoffs but the one at 55 years being statistically significant. Asterisks represent statistically significant differences $(p$-values $<0.05)$. 
A

C
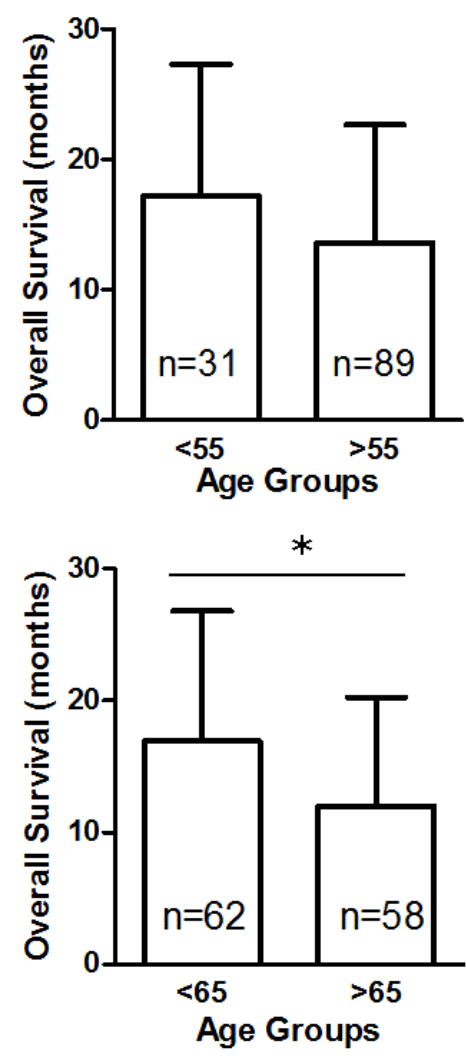

B

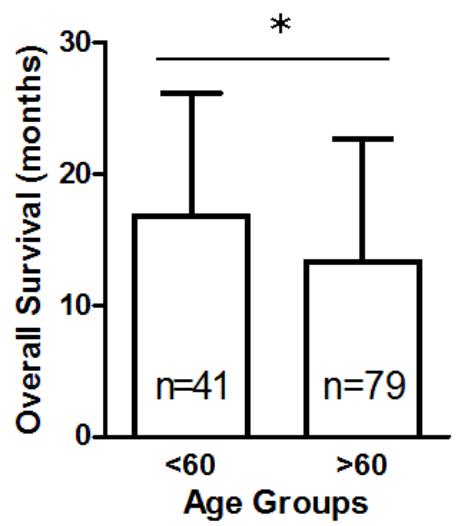

$\mathrm{D}$

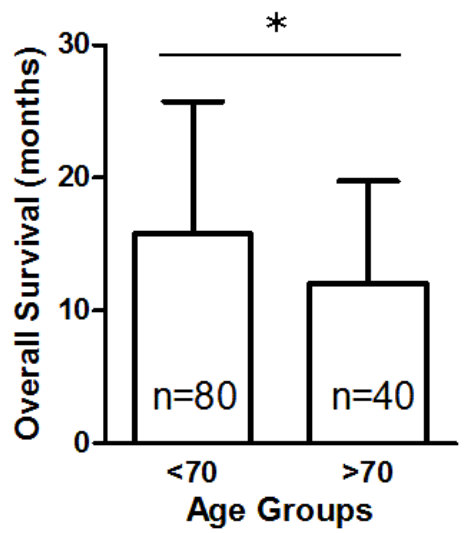

Figure 3.5: Younger age is a significant prognostic factor in GBM II. Mean overall survival of patients diagnosed with $G B M$ divided into age groups at cutoffs at $55(\boldsymbol{A}), 60(\boldsymbol{B})$, $65(\boldsymbol{C})$ and 70 years $(\boldsymbol{D})$. Bars represent mean values with standard deviation. Asterisks represent statistically significant differences ( $p$-values $<0.05)$.

\subsubsection{KPS}

Similar to the cutoff at 60 years of age, a KPS of $70 \%$ or higher is used to decide further adjuvant treatment. It serves as an indicator for the general state of health required to endure the strain and adverse effects of chemotherapy. Its prognostic role is also well established (see section 1.2.1). In this study patients presented with a mean KPS of $72.08 \%$ at the time of diagnosis while 85 of 120 patients held a KPS of $70 \%$ or higher $(70.83 \%)$. Figure 3.6 shows the correlation of higher KPS with increasing overall survival of the surveyed GBM population ( $p=0.0006$, slope significantly not zero). 

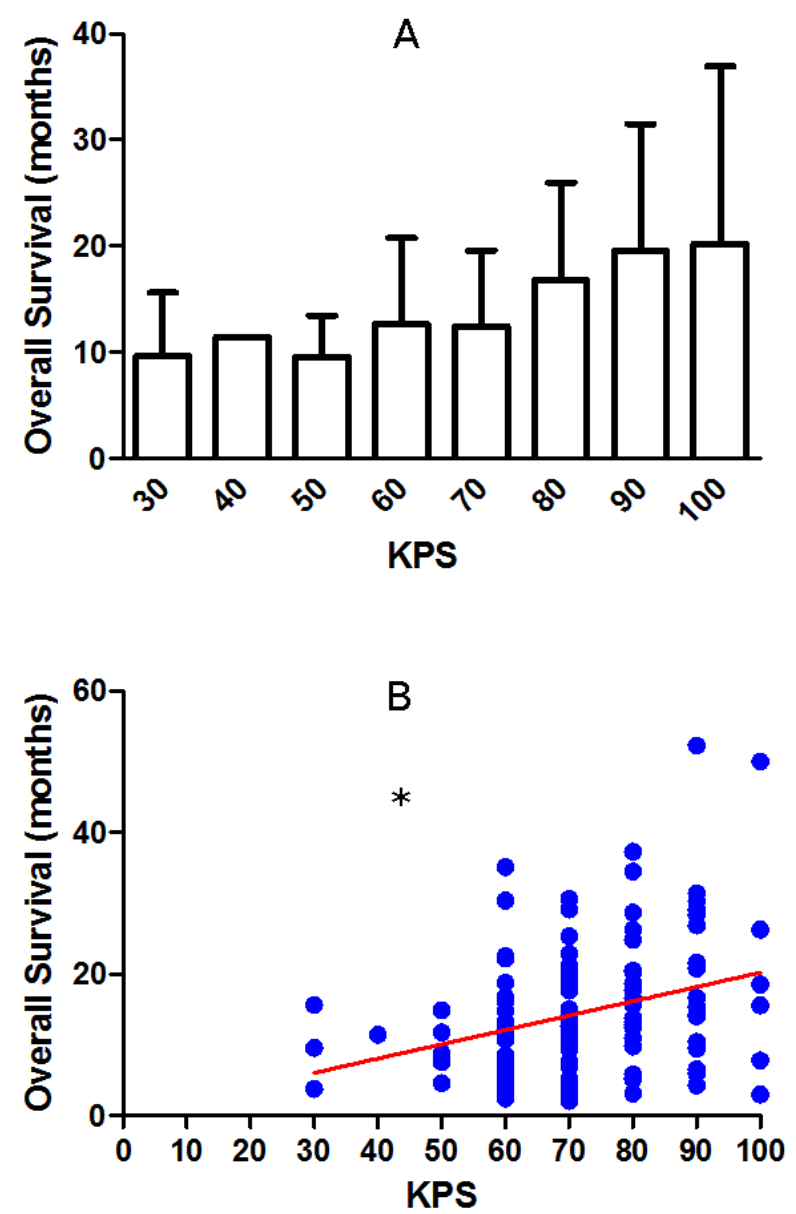

Figure 3.6: Higher Karnofsky scores are associated with longer overall survival in GBM I. A: Bar graphs illustrating the mean overall survival with standard deviation for each KPS score. B: Scatterplot depicting KPS scores of individual patients according to overall survival. The red line indicates a positive correlation of KPS with prolonged overall survival (slope significantly not zero, $p=0.0006$ ). Bars represent mean values with standard deviation. Asterisks represent statistically significant differences ( $p$-values $<0.05)$.

In order to investigate the prognostic role of the clinical status, the patient collective was divided into two groups according to the KPS at diagnosis. The cutoffs for this division were set at $60 \%, 70 \%, 80 \%$ and $90 \%$. The difference in overall survival at each cutoff is displayed in figure 3.7 and 3.8 as Kaplan-Meier curves and bar graphs. At all cutoffs the patient group with the higher KPS showed a tendency for longer mean overall survival.

Patients holding a KPS of $60 \%$ or more had a mean overall survival of 14.92 months compared to 9.78 months in patients with lower Karnofsky Performance Scores (patients requiring at least frequent medical care and assistance). This apparent difference failed to reach statistical significance $(\mathrm{p}=0.0824)$. The cutoff at $70 \%$ closely missed statistical significance as well $(\mathrm{p}=0.0539)$. Patients who were able to care for themselves (KPS of $70 \%$ or higher) lived for 15.61 months on average, while lower KPS 
resulted in a mean overall survival of 11.92 months. It is worth mentioning that both cutoffs, at $60 \%$ and $70 \%$, showed a statistical trend towards longer overall survival for higher KPS scores.

A KPS of $80 \%$ or higher was associated with a significantly increased mean overall survival of 18.30 months compared to lower scores which had a mean overall survival of 12.19 months $(\mathrm{p}=0.0020)$. The cutoff at $90 \%$ was also statistically significant $(\mathrm{p}=0.0278)$. Individuals who showed only mild or no signs of disease (KPS 90 or $100 \%$ ) lived for 19.70 months on average after diagnosis of GBM, whereas Karnofsky scores of $80 \%$ and lower showed a mean overall survival of 13.24 months. Multivariate analysis revealed KPS to be a highly significant prognostic factor in glioblastoma $(\mathrm{p}=0.00165$, see section 3.4).


Figure 3.7: Higher Karnofsky scores are associated with longer overall survival in GBM II. Difference in overall survival displayed as Kaplan-Meier curves. The cutoffs were chosen at $60 \%(\boldsymbol{A}), 70 \%(\boldsymbol{B}), 80 \%(\boldsymbol{C})$ and $90 \%(\boldsymbol{D})$. Higher Karnofsky scores are associated with longer overall survival. Asterisks represent statistically significant differences $\left({ }^{*}\right.$ p-values $<0.05$, ${ }^{* *}$ p-values $\left.<0.005\right)$. 
A

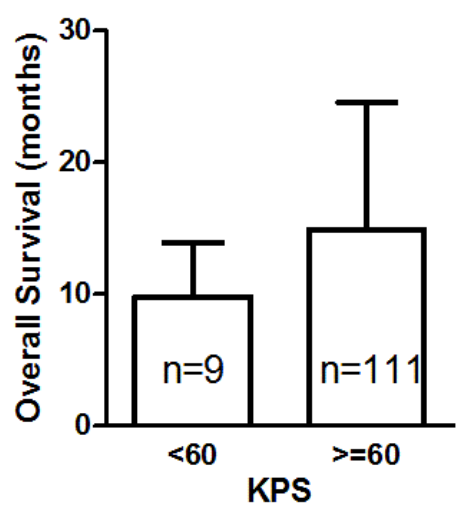

C

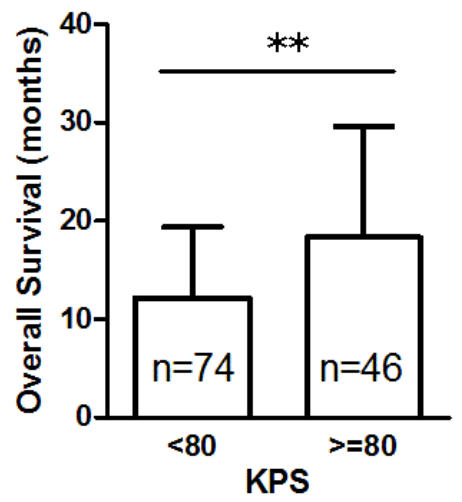

B

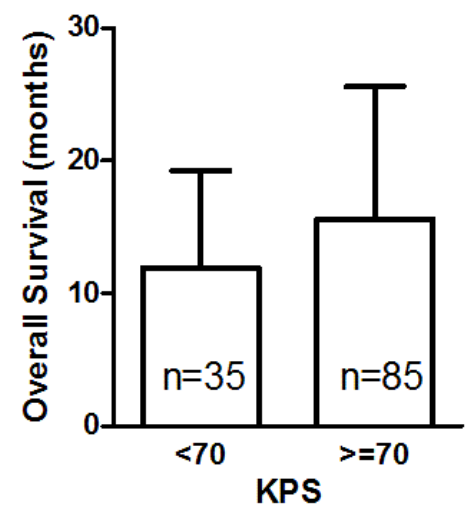

D

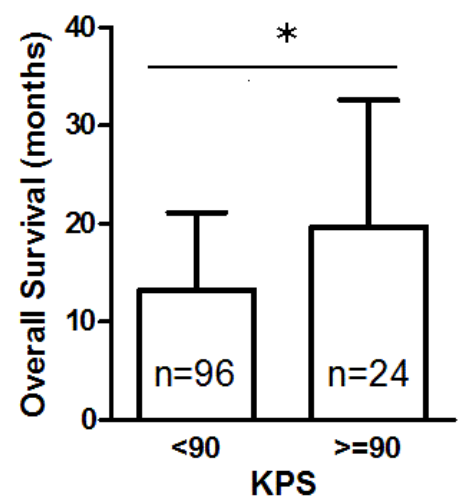

Figure 3.8: Higher Karnofsky scores are associated with longer overall survival in GBM III. Difference in mean overall survival with cutoffs chosen at Karnofsky scores of $60 \%(\boldsymbol{A}), 70 \%(\boldsymbol{B}), 80 \%(\boldsymbol{C})$ and $90 \%(\boldsymbol{D})$. Bars represent mean values with standard deviation. Asterisks represent statistically significant differences $\left({ }^{*} p\right.$-values $<0.05$, ${ }^{* *} p$ values $<0.005)$.

\subsubsection{Extent of Resection}

The extent of resection is known to play an important prognostic role. As a thresholds, a cytoreduction of 78-98\% has to be achieved in order to prolong the overall survival (see chapter 1.1.4). In this study 119 of 120 patients received surgical treatment. In 93 of 120 cases gross total resection was attained, while 26 individuals received a subtotal resection and one GBM was merely biopsied. The mean overall survival of patients that underwent gross total resection was longer than subtotally resected or biopsied cases with 15.26 compared to 12.03 months, respectively. However, within this patient collective the difference in overall survival was not statistically significant in the Kaplan-Meier analysis (Gehan's Wilcoxon test, $\mathrm{p}=0.1583$ ). The clinical status at the time of diagnosis (KPS) did not show a considerable difference between the two groups (gross total resection $72.37 \%$, subtotal resection or biopsy $71.11 \%, \mathrm{p}=0.7012$ ). With 59.21 years the 27 patients undergoing partial tumor resection or biopsy tended 
to be slightly younger at the time of diagnosis in comparison to the mean age of 63.15 years of individuals receiving gross total resection. However, statistical significance was not reached $(\mathrm{p}=0.1764)$ (see figure 3.9). Multivariate analysis showed a mentionable trend towards significance regarding EOR being an independent prognostic factor $(\mathrm{p}=0.0601$, see section 3.4).
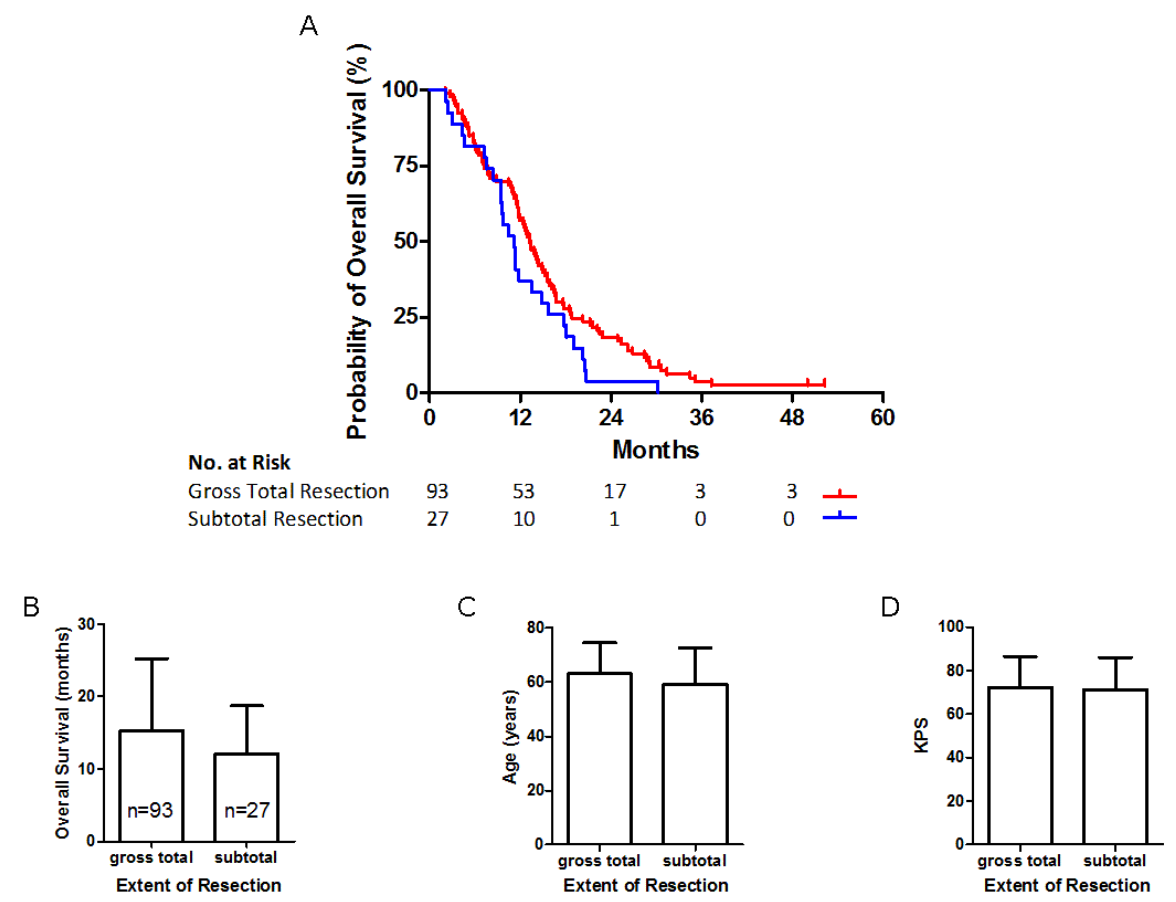

Figure 3.9: Gross total resection shows a tendency towards longer overall survival. Panel $\boldsymbol{A}$ displays the prognostic role of gross total resection compared to subtotal resection or biopsy in glioblastoma patients as a Kaplan-Meier curve. The lower graphs show mean overall survival $(\boldsymbol{B})$ as well as age $(\boldsymbol{C})$ and $K P S(\boldsymbol{D})$ at the time of diagnosis. Gross total resection led to longer overall survival while KPS and age showed similar values compared to partially resected and biopsied patients. However, no statistical significance was reached ( $p=0.1583, p=0.7012$ and $p=0.1764$, respectively). However, multivariate analysis identified a trend of gross total resection towards significance regarding its independent prognostic value $(p=0.0601$, see chapter 3.4). Bars represent mean values with standard deviation. Asterisks represent statistically significant differences ( $p$-values $<0.05)$. 


\subsubsection{Chemotherapy}

During the last decade TMZ became the established adjuvant chemotherapeutic treatment for glioblastoma multiforme, due to its superior effect on overall survival. ACNU is also an alkylating agent that was widely used before the initiation of TMZ. BCNU is applied as a local alkylating agent additionally to TMZ. Studies produced contradictory results regarding its prognostic benefit (see chapter 1.1.4). All 120 patients received adjuvant chemotherapy with alkylating agents. The difference in overall survival as well as age and KPS at the time of diagnosis are displayed in figure 3.10. In 97 cases temozolomide was administered. This led to a mean overall survival of 14.06 months. On average, individuals of this subgroup were 63.18 years old and held a KPS of $71.86 \%$ at the time of diagnosis. Patients treated with ACNU ( $n=15)$ showed a similar mean overall survival and KPS at the time of diagnosis (14.55 years and $70.67 \%, \mathrm{p}=0.7272$ and $\mathrm{p}=0.7164$, respectively), while the average age was slightly lower without statistical significance $(59.88$ years, $\mathrm{p}=0.3135)$. 8 individuals received BCNU additionally to TMZ in form of a Gliadel ${ }^{\circledR}$ wafer that was placed into the resection cavity. The mean overall survival was over 6 months longer than in the subgroup treated with TMZ alone (20.29 months), although, failing statistical significance $(\mathrm{p}=0.0958)$. At the time of diagnosis this subgroup was younger (mean age 55.63 years compared to 63.18 years, $\mathrm{p}=0.1172$ ) and showed a tendency towards better clinical status (mean KPS $77.50 \%$ compared to $71.86 \%, \mathrm{p}=0.07665$ ). However, both differences failed to reach statistical significance. Overall, it can be stated that there is a statistical trend towards longer overall survival of patients that received additional BCNU. 




Figure 3.10: No differential prognostic impact among alkylating chemotherapeutic agents in $\boldsymbol{G B M}$. Panel $\boldsymbol{A}$ displays the difference in overall survival of GBMs treated with TMZ, ACNU and TMZ plus Gliadel ${ }^{\circledR}$ wafer (BCNU) as a Kaplan-Meier curve. The lower graphs depict the overall survival $(\boldsymbol{B})$ as well as age $(\boldsymbol{C})$ and $K P S(\boldsymbol{D})$ at diagnosis in bar graphs. BCNU seems to lead to longer overall survival, although failing to reach statistical significance ( $p=0.0958)$. Patients receiving BCNU tended to be younger and held a slightly higher KPS at the time of diagnosis ( $p=0.1172$ and $p=0.07665$, respectively). Bars represent mean values with standard deviation. Asterisks represent statistically significant differences (p-values $<0.05)$. 


\subsection{Prognostic Role of Immunohistochemical Markers}

Immunohistochemical markers reflect distinct molecular and biological properties of the tumor cells (e.g. proliferation, differentiation etc.) and could therefore be instrumental in the development of targeted individual therapies. However, with a few exceptions, the prognostic value and clinical significance of immunohistochemical markers in glioblastoma remains unclear. In this study the expression levels of several immunohistochemical markers were investigated regarding their prognostic impact on patients diagnosed with glioblastoma multiforme and their possible correlation with clinical status (KPS) and age at the time diagnosis. IDH-1 and TP53 mutation and the expression levels of NogoA, Ki67, OLIG2 and GFAP were subject to this study.

\subsubsection{IDH-1 Mutation}

IDH-1 mutations are frequently present in low-grade gliomas (see figure 3.11). Its role in glioblastoma has recently been established as a specific marker for secondary GBM, a glioblastoma subgroup with younger age and better prognosis (see chapter 1.2.4 and 1.2.5). Therefore, all 120 samples underwent analysis for IDH-1 mutation and its clinical impact.

Positive immunoreactivity of a single cell manifested itself as brown perinuclear coloring. The controls utilized for the immunohistochemical staining of the IDH-1 mutation are shown in figure 3.11. Diffuse and anaplastic oligodendrogliomas (WHO grade II and III, respectively) served as positive controls. In figure 3.12 examples of positive and negative glioblastoma tissue can be seen.

Of 120 patients 6 cases displayed a mutation of IDH-1 on immunohistochemical staining (5\%). This small subgroup held a slightly higher mean KPS and was younger at the time of diagnosis (76.67\% and 47.68 years) compared to their wildtype counterparts (71.84\% and 63.03 years). The variation in KPS did not reach statistical significance $(\mathrm{p}=0.637)$, while the difference in age at diagnosis almost did ( $\mathrm{p}=0.05063)$. The mean overall survival was also higher, yet not significantly (17.95 compared to 14.36 months, $\mathrm{p}=0.8276$ ) (see figure 3.13 ). 




Figure 3.11: IDH-1 mutation controls. 400-fold magnification of the controls: cerebellum $(\boldsymbol{A})$, hippocampus $(\boldsymbol{B})$, cerebral melanoma metastasis $(\boldsymbol{C})$, diffuse oligodendroglioma (WHO grade II) (D), anaplastic oligodendroglioma (WHO grade III) (E) and pilocytic astrocytoma (WHO grade I) (F). Diffuse and anaplastic oligodendroglioma served as positive controls for IDH-1 mutation.

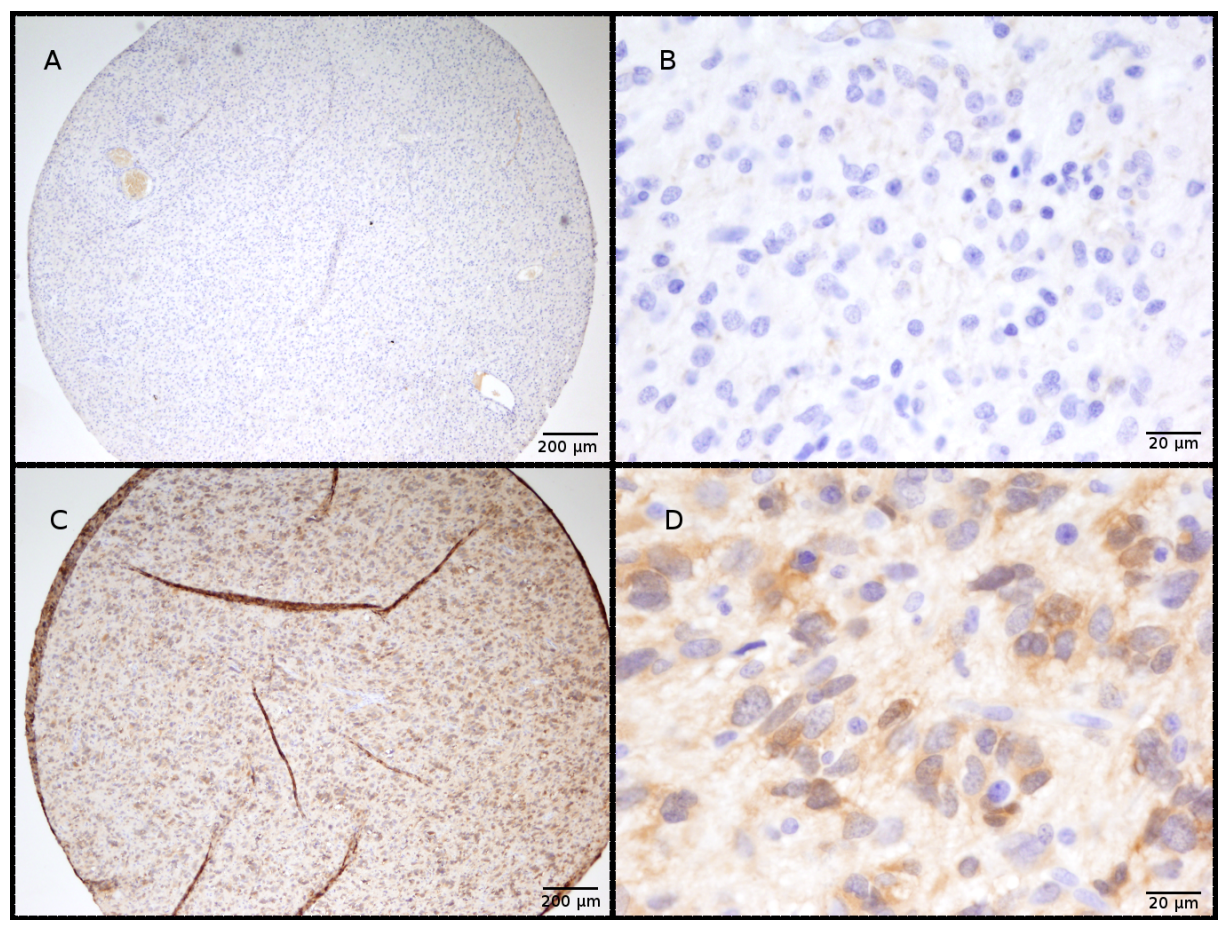

Figure 3.12: IDH-1 mutations in $\boldsymbol{G B M}$. Negative ( $\boldsymbol{A}$ and $\boldsymbol{B}$ ) and positive tumor tissue samples $(\boldsymbol{C}$ and $\boldsymbol{D})$ with the typical brown perinuclear coloring (100- and 400-fold magnification). 




Figure 3.13: Trend towards younger age of patients with IDH-1 mutations in GBM. Panel $\boldsymbol{A}$ shows a Kaplan-Meier curve of GBMs with and without IDH-1 mutations. The lower panels show the differences in overall survival $(\boldsymbol{B})$, age $(\boldsymbol{C})$ and $K P S(\boldsymbol{D})$ in bar graphs. Patients holding an IDH-1 mutation show a trend towards younger age that barely missed statistical significance $(p=0.05063)$. The observed longer overall survival and higher KPS at the time of diagnosis of IDH-1-mutated glioblastomas did not reach statistical significance $(p=0.8276$ and $p=0.637)$. Bars represent mean values with standard deviation. Asterisks represent statistically significant differences ( $p$-values $<0.05$ ). 


\subsubsection{NogoA}

NogoA is an established positive prognostic marker in oligodendrogliomas. Two recent studies revealed a subgroup of approximately $20 \%$ of GBMs with highly increased expression levels of this protein. However, only small groups of 30 and 29 GBMs were evaluated (see chapter 1.2.6). The patient cohort of this study is big enough to attain more profound results regarding the distribution and prognostic impact of NogoA in GBM.

Immunohistochemical staining for NogoA was regarded as positive if a cell displayed brown perinuclear coloring. Some samples showed a very weak homogenous coloring throughout the whole sample. This was regarded as background staining and was not considered as positive immunoreactivity. Diffuse and anaplastic oligodendroglioma (WHO grade II and III, respectively) as well as pilocytic astrocytoma (WHO grade I) served as positive controls (see figure 3.14).

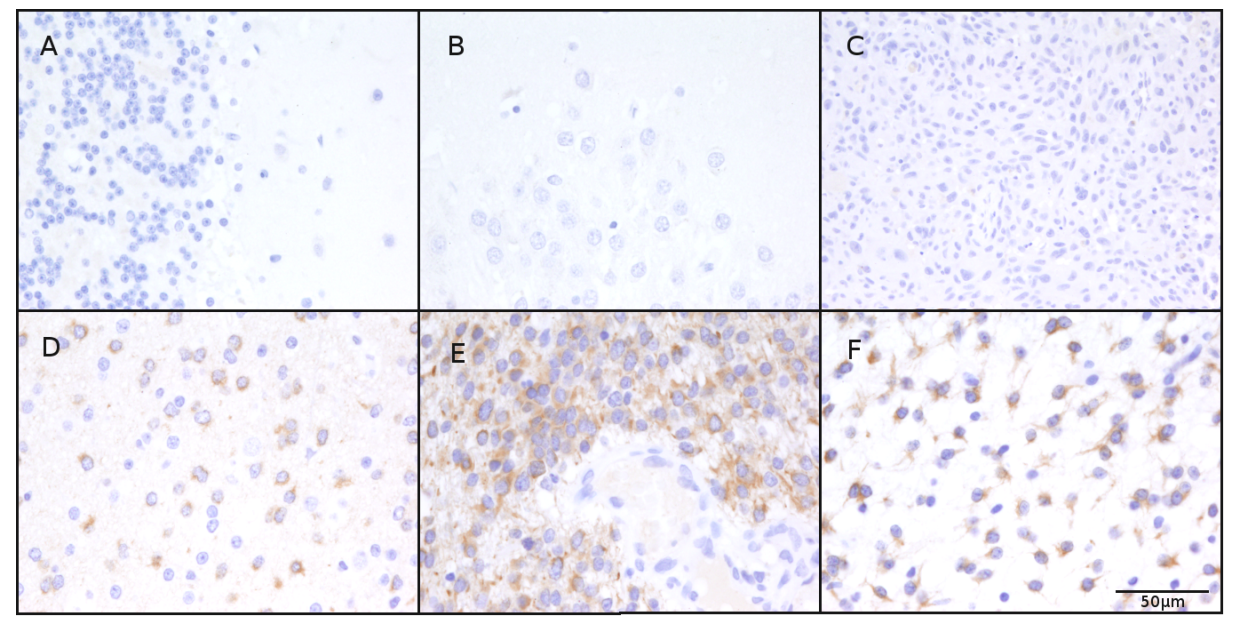

Figure 3.14: NogoA controls. 400-fold magnification of the controls: cerebellum (A), hippocampus $(\boldsymbol{B})$, cerebral melanoma metastasis $(\boldsymbol{C})$, diffuse oligodendroglioma (WHO grade II) (D), anaplastic oligodendroglioma (WHO grade III) (E) and pilocytic astrocytoma (WHO grade I) (F).Diffuse and anaplastic oligodendroglioma and pilocytic astrocytoma served as positive controls for NogoA staining. Positive immunoreactivity resulted in brown coloring in a perinuclear pattern.

A scoring system for NogoA immunohistochemical staining was established in order to quantify the extent of immunopositivity of each sample. The sample received +++ if the majority of the cells were NogoA positive $(>50 \%),++$ if several cells were positive (5-50\%) and + if few positive cells were scattered throughout the sample $(<5 \%)$. The score $+/$ - was given to samples that displayed only very few single positive cells (see figure 3.15). 5 samples showed high expression rates of over $50 \%$ $(+++)$ of the tumor cells $(4.17 \%)$. Another 20 tumor samples $(16.67 \%)$ revealed many immunopositive cells while the majority of the tumor tissue remained unstained $(++)$. The rest of the samples held none or merely a few scattered NogoA-positive tumor 
cells $(79.17 \%,+$ or $+/-$ ) (see figure 3.16). For statistical analysis of the prognostic role of NogoA expression, the patient collective was divided into two groups with higher and lower expression levels with chosen cutoffs at $+/-,+$ and ++ .

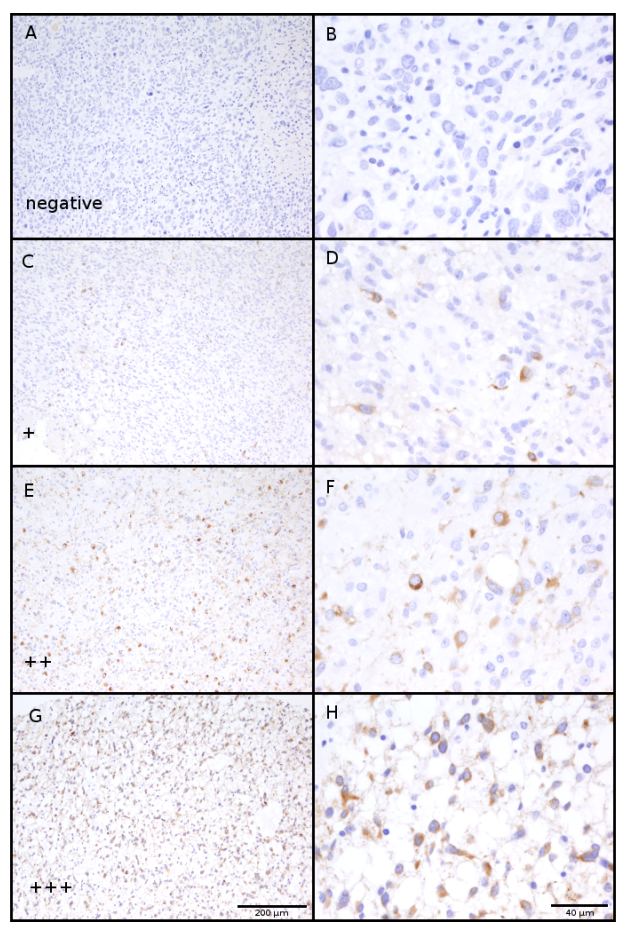

Figure 3.15: Quantification of NogoA immunopositivity. The first row (A and B) shows a negatve sample (-), the second ( $\boldsymbol{C}$ and $\boldsymbol{D})$ only scattered positive cells $(+) . A$ sample was scored with $+/-$ if only very few cells were detected in the sample. The third sample from above ( $\boldsymbol{E}$ and $\boldsymbol{F}$ ) holds many NogoA-positive cells $(++)$ but does not reach positivity of the majority of the cells (over 50\%) like the sample displayed in the fourth row $(+++)$ ( $\boldsymbol{G}$ and $\boldsymbol{H})$ (100-fold magnification on the left and 400-fold on the right).



Figure 3.16: Distribution of NogoA immunopositivity.

The first cutoff resulted in 29 samples with expression levels below or equal to +/-, meaning very few scattered, single immunopositive cells or none, and 91 samples with 
higher expression levels. Tumor samples that scored $+/$ - with one sample and + with the other were scored as above $+/-$. Age and KPS at diagnosis were quite similar for both groups with 63.40 years and $71.38 \%$ compared to 61.90 years and $72.31 \%$ for lower and higher expression levels, respectively (Age: $\mathrm{p}=0.5678$, KPS: $\mathrm{p}=0.7657$ ). The 29 samples with very few or none immunoreactivity to NogoA showed a mean overall survival of 16.41 months while higher expression levels resulted in an average of 13.94 months. However, the difference was not significant $(\mathrm{p}=0.5420)$ (see figure $3.17)$.

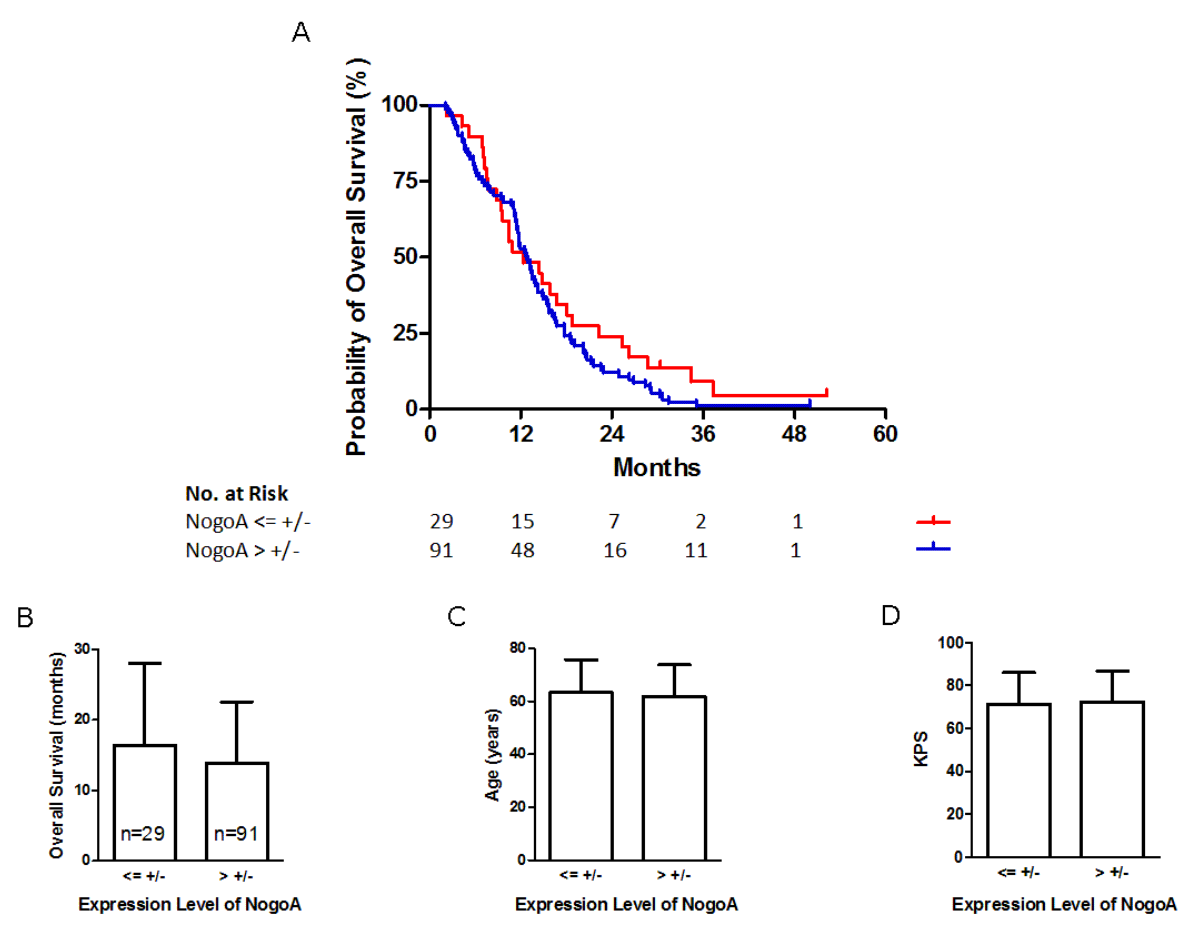

Figure 3.17: NogoA cutoff at $+/$ - without significant differences. Illustration of overall survival as Kaplan-Meier curve $(\boldsymbol{A})$ and depiction of overall survival $(\boldsymbol{B})$, age at diagnosis $(\boldsymbol{C})$ and KPS $(\boldsymbol{D})$ as bar graphs. Age and KPS at diagnosis as well as overall survival did not reveal a striking difference between the two groups ( $p=0.5678, p=0.7657$ and $p=0.5420$, respectively). Bars represent mean values with standard deviation. Asterisks represent statistically significant differences ( $p$-values $<0.05)$.

The next cutoff divided the samples into 48 with expression levels below + and 72 equal or above + . This cutoff was similar to the first with the difference that tissue samples with one sample displaying $+/$ - and the other + were scored as below + overall and therefore 19 more cases fell below the cutoff. Average age and KPS at diagnosis were 62.45 years and $70.00 \%$ compared to 62.14 years and $73.47 \%$ for lower and higher expression levels, respectively. These differences were not statistically significant (Age: $\mathrm{p}=0.8888$, KPS: $\mathrm{p}=0.2085)$. Expression levels below + showed a slightly longer mean overall survival with 15.75 years compared to 13.73 years of higher expression levels, yet failing to reach statistical significance $(\mathrm{p}=0.2708)$ (see figure 3.18). 




Figure 3.18: NogoA cutoff at + without significant differences. Illustration of overall survival as Kaplan-Meier curve $(\boldsymbol{A})$ and depiction of overall survival $(\boldsymbol{B})$, age at diagnosis $(\boldsymbol{C})$ and $K P S(D)$ as bar graphs. Expression levels of NogoA above or equal + showed a slightly higher KPS at diagnosis and a shorter mean overall survival. However, in both cases statistical significance was not reached ( $p=0.2085$ and $p=0.2708$, respectively). Age at diagnosis was similar for both groups $(p=0.8888)$. Bars represent mean values with standard deviation. Asterisks represent statistically significant differences (p-values $<0.05)$.

The last cutoff for NogoA was set at ++ , resulting in 95 samples with an expression level below ++ and 25 above or equal ++ . This divided the sample population into a group with immunopositivity of a large portion of the tumor cells (above or equal ++ ) and samples with scattered few, very few or no NogoA-positive cells (below ++ ). There was no difference in mean age at diagnosis (62.06 compared to 63.03 years, $\mathrm{p}=0.7$ ) and overall survival (14.52 compared to 14.59 months, $\mathrm{p}=0.6961$ ) of the two groups (below ++ and above or equal ++ , respectively). Samples with higher NogoA expression showed a slightly higher KPS at diagnosis, however, without statistical significance (75.20 compared to $71.26 \%, \mathrm{p}=0.18$ ) (see figure 3.19 ). 


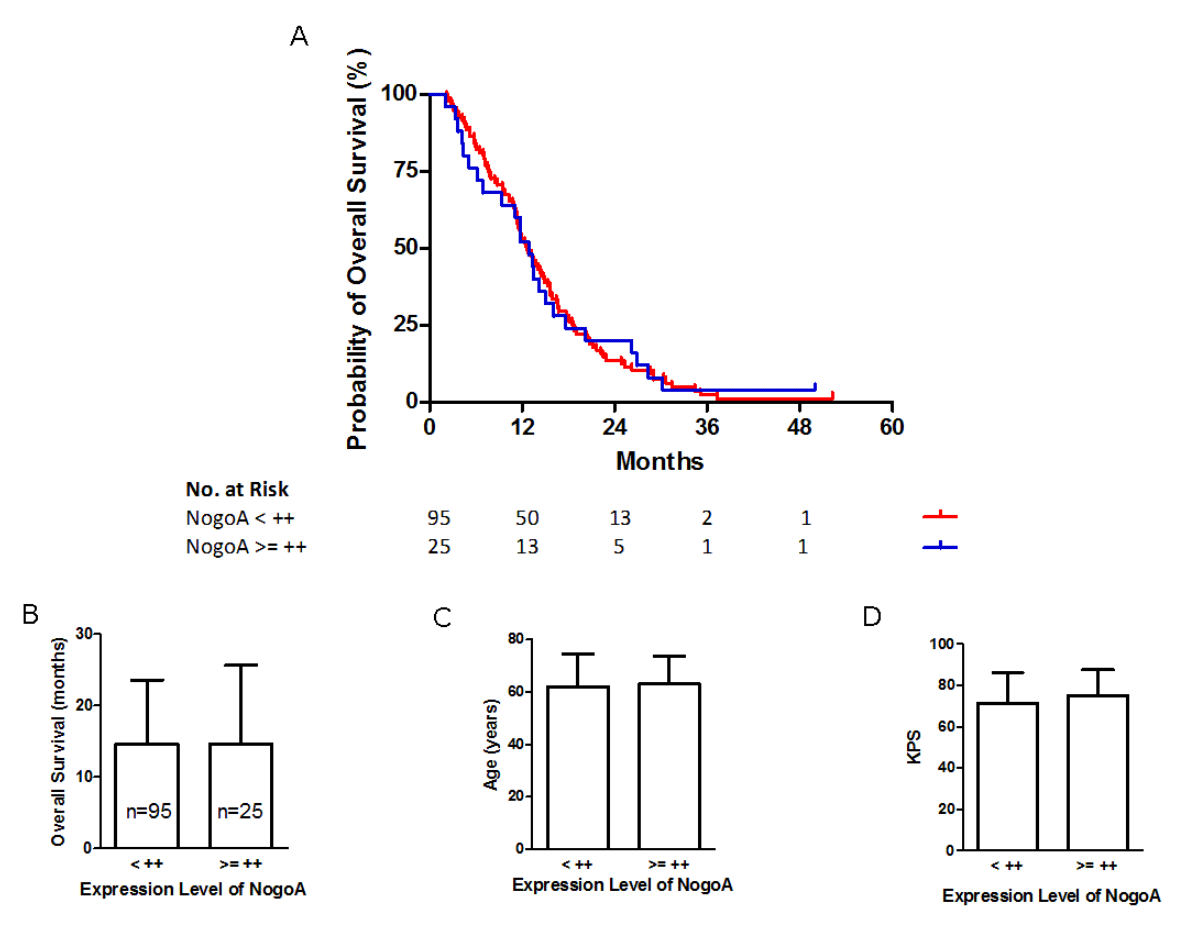

Figure 3.19: NogoA cutoff at ++ without significant differences. Illustration of overall survival as Kaplan-Meier curve $(\boldsymbol{A})$ and depiction of overall survival $(\boldsymbol{B})$, age at diagnosis $(\boldsymbol{C})$ and KPS $(\boldsymbol{D})$ as bar graphs. Overall survival, clinical status and age at the time of diagnosis showed similar results for both groups $(p=0.6961, p=0.18$ and $p=0.7$, respectively). Bars represent mean values with standard deviation. Asterisks represent statistically significant differences ( $p$-values $<0.05)$. 


\subsubsection{OLIG2}

OLIG2 is a transcription factor that drives oligodendrocyte differentiation and was also found to play a role in oligodendrocytic and astrocytic tumor development. Research has focused primarily on anaplastic oligodendrogliomas and astrocytomas. Its role in glioblastoma is still unclear (see chapter 1.2.6). Therefore one part of this study focuses on the impact of OLIG2 in GBM and its clinical significance. Increased expression of OLIG2 can be illustrated immunohistochemically as brown nuclear coloring. Among the controls oligodendrocytes of hippocampus and cerebellum tissue were immunopositive while diffuse and anaplastic oligodendroglioma (WHO grade II and III, respectively) as well as pilocytic astrocytoma (WHO grade I) presented strong positive controls. Melanoma metastasis was immunonegative (see figure 3.20). A wide range of immunopositivity rates within the sample collective was observed (see figure 3.21). Only 4 samples did not reveal any expression of OLIG2 and 2 tumors had a positivity rate of below $1 \%$ while the rest of the samples ranged from 5 to $70 \%$. Figure 3.22 illustrates some examples of GBM tissue samples with immunopositivity rates of $5 \%, 10 \%, 40 \%$ and $70 \%$.



Figure 3.20: OLIG2 controls. 400-fold magnification of the controls: cerebellum (A), hippocampus $(\boldsymbol{B})$, cerebral melanoma metastasis $(\boldsymbol{C})$, diffuse oligodendroglioma (WHO grade II) (D), anaplastic oligodendroglioma (WHO grade III) (E) and pilocytic astrocytoma (WHO grade I) (F). Except for melanoma metastasis, all controls showed positivity for OLIG2 to some extent but diffuse and anaplastic oligodendrogliomas and pilocytic astrocytoma served as strong positive controls. In hippocampal and cerebellar tissue only oligodendrocytes were weakly immunopositive. 
Identification of Prognostically Relevant Cellular Markers of Differentiation in Glioblastoma

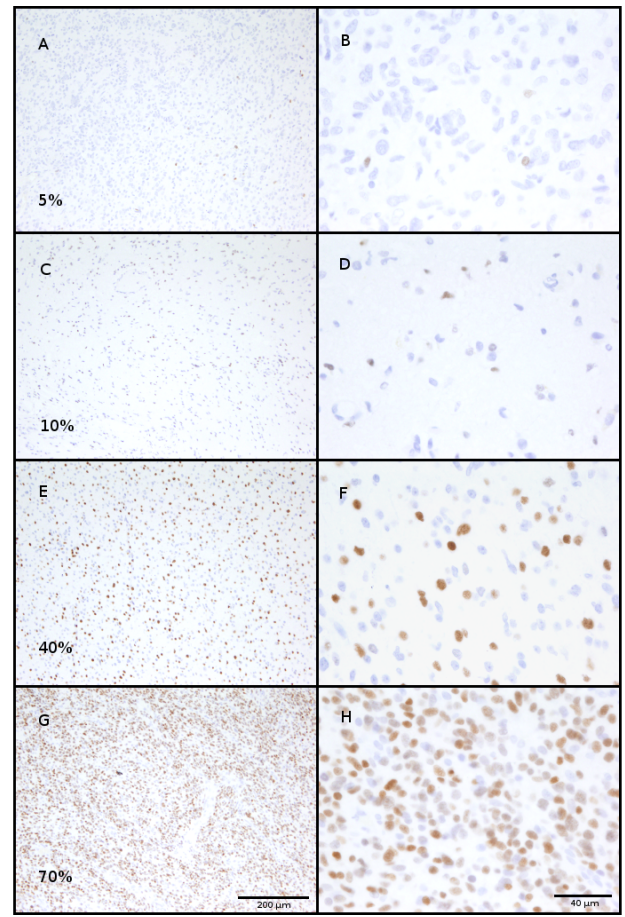

Figure 3.21: Examples of different OLIG2 expression levels. A and B show a tumor sample with about $5 \%$ immunopositive cells while the $\boldsymbol{C}$ and $\boldsymbol{D}$ show a sample that reaches as high as 10\%. The third and the bottom row depict samples with high immunopositivity rates of $40 \%$ ( $\boldsymbol{E}$ and $\boldsymbol{F})$ and $70 \%(\boldsymbol{G}$ and $\boldsymbol{H})$, respectively (100-fold magnification on the left and 400-fold on the right).

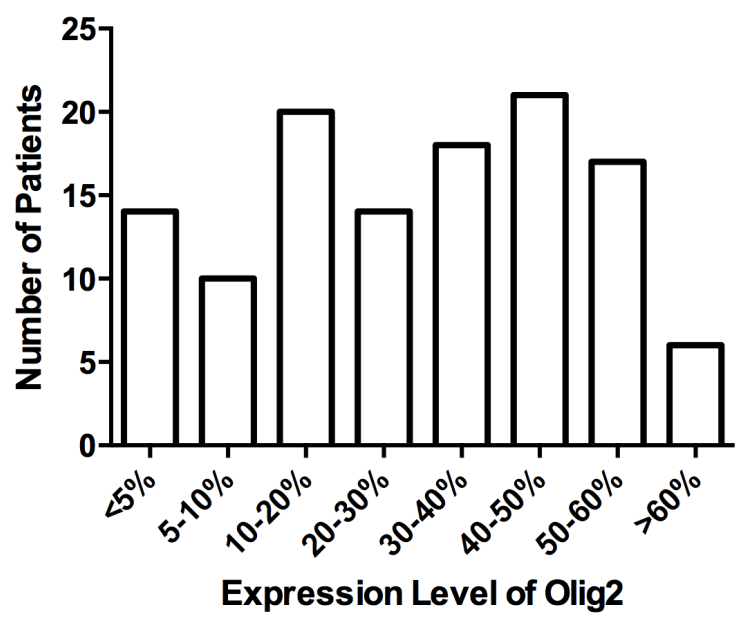

Figure 3.22: Distribution of OLIG2 expression throughout the sample collective. 
Then we analyzed overall survival, age and KPS of patients with different percentages of OLIG2 positive cells. For this purpose the patients were divided into groups including distinct cutoff points at 5, 10, 20, 30, 40 and 50\%. First we analyzed the group with a cutoff set at $5 \%$ immunopositivity (see figure 3.23 ). This division resulted in 14 patients with expression rates below $5 \%$ who were slightly younger and held a lower clinical status at the time of diagnosis (58.25 years and $66.43 \%$ ) compared to the remaining 106 individuals (62.79 years and 72.83\%). These differences did not reach statistical significance (Age: $p=0.3217$, KPS: $p=0.2048$ ). There was a longer mean overall survival in favor of tumor samples with immunopositive rates below 5\%, however failing statistical significance (18.40 months compared to 14.03 months, $\mathrm{p}=0.2566$ ).

A

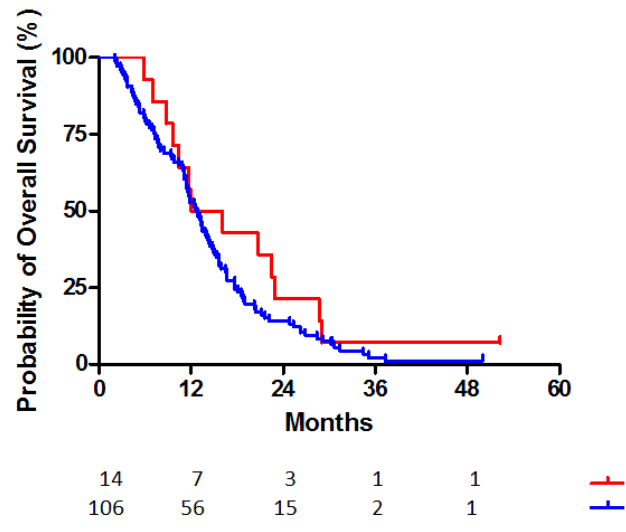

B

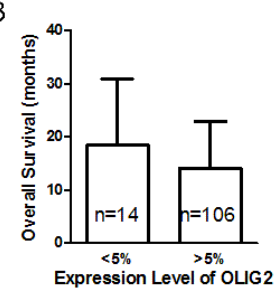

C



D

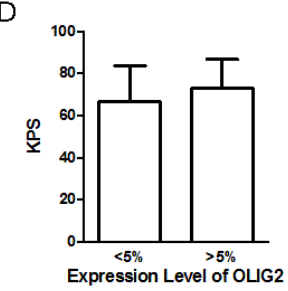

Figure 3.23: Longer mean overall survival of OLIG2 expression below $5 \%$ failed statistical significance. Illustration of overall survival as Kaplan-Meier curve (A) and depiction of overall survival $(\boldsymbol{B})$, age at diagnosis $(\boldsymbol{C})$ and $K P S(\boldsymbol{D})$ as bar graphs. Patients with immunopositive rates below 5\% were slightly younger at the time of diagnosis and showed a lower clinical status, however, both without statistical significance ( $p=0.3217$ and $p=0.2048$, respectively). These individuals showed no statistically significant difference in overall survival $(p=0.2566)$. Bars represent mean values with standard deviation. Asterisks represent statistically significant differences $(p$-values $<0.05)$. 
24 GBM samples showed less than $10 \%$ of immunopositive cells (see figure 3.24). There was no difference in age at diagnosis in comparison to the other 96 samples which held higher OLIG2 expression rates (62.31 compared to 62.25 years, $\mathrm{p}=0.9865$ ). However, similar to the results of the cutoff at $5 \%$, the clinical status of low expression samples was slightly lower while the mean overall survival was longer $(68.75 \%$ compared to $72.97 \%$ and 17.60 compared to 13.77 months). However, the differences in overall survival and KPS did not reach statistical significance $(\mathrm{p}=0.1438$ and $\mathrm{p}=0.216$, respectively).

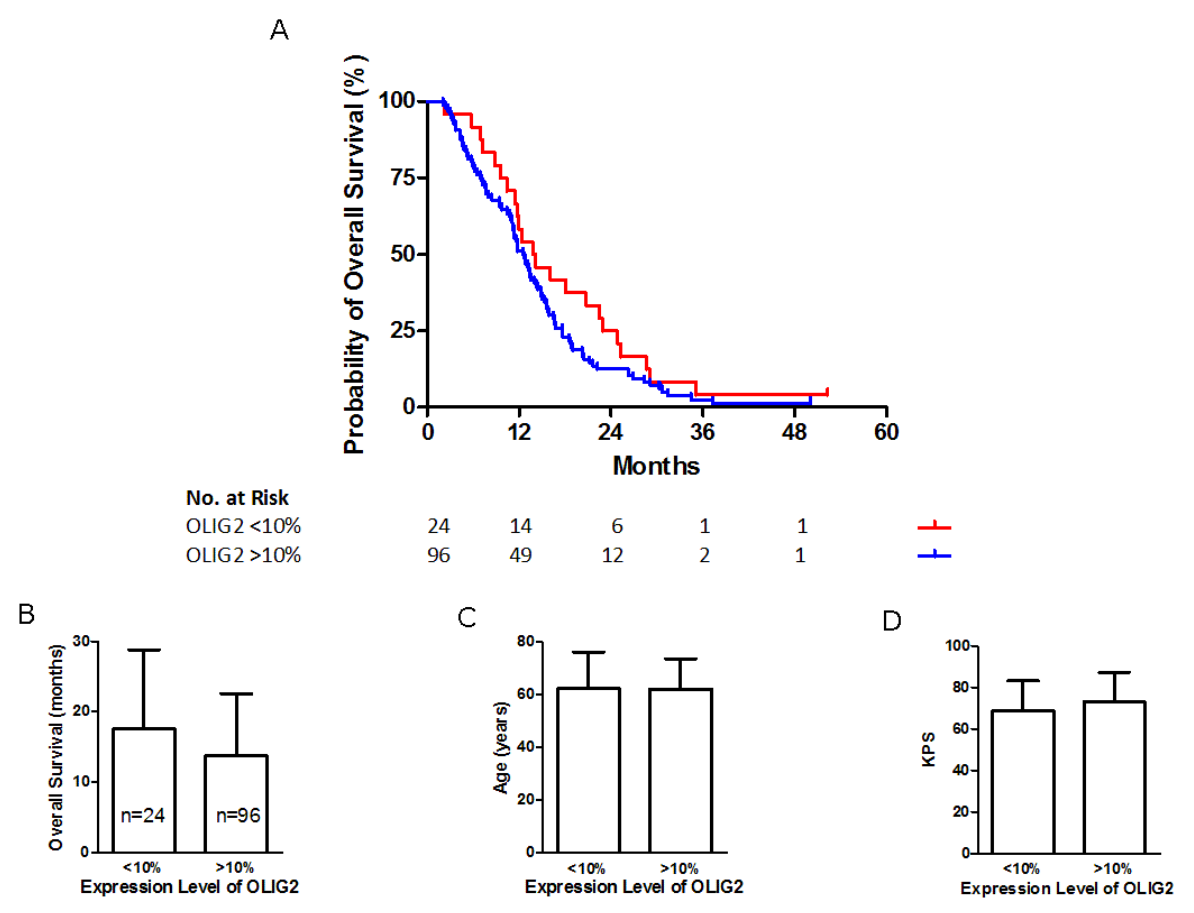

Figure 3.24: Longer mean overall survival of OLIG2 expression below 10\% failed statistical significance. Illustration of overall survival as Kaplan-Meier curve (A) and depiction of overall survival $(\boldsymbol{B})$, age $(\boldsymbol{C})$ at diagnosis and KPS $(\boldsymbol{D})$ as bar graphs. There is a longer overall survival of patients with immunopositive rates below 10\%, nonetheless without statistical significance $(p=0.1438)$. KPS and age at the time of diagnosis were without significant difference between the two groups ( $p=0.216$ and $p=0.9865$, respectively). Bars represent mean values with standard deviation. Asterisks represent statistically significant differences ( $p$-values <0.05).

A cutoff chosen at an immunopositivity rate of $20 \%$ led to the division into 44 tumor samples with lower and 76 GBMs with higher immunopositive rates (see figure 3.25). Age and KPS at the time of diagnosis revealed no considerable difference between lower and higher OLIG2 expression (61.56 years and $72.95 \%$ compared to 62.67 years and $71.58 \%$ with $\mathrm{p}=0.6292$ and $\mathrm{p}=0.6189$, respectively). However, mean overall survival of patients with samples that held less than $20 \%$ immunopositive cells for OLIG2 were longer, but without statistical significance as well (15.89 months compared to 13.75 months, $\mathrm{p}=0.2952$ ). 

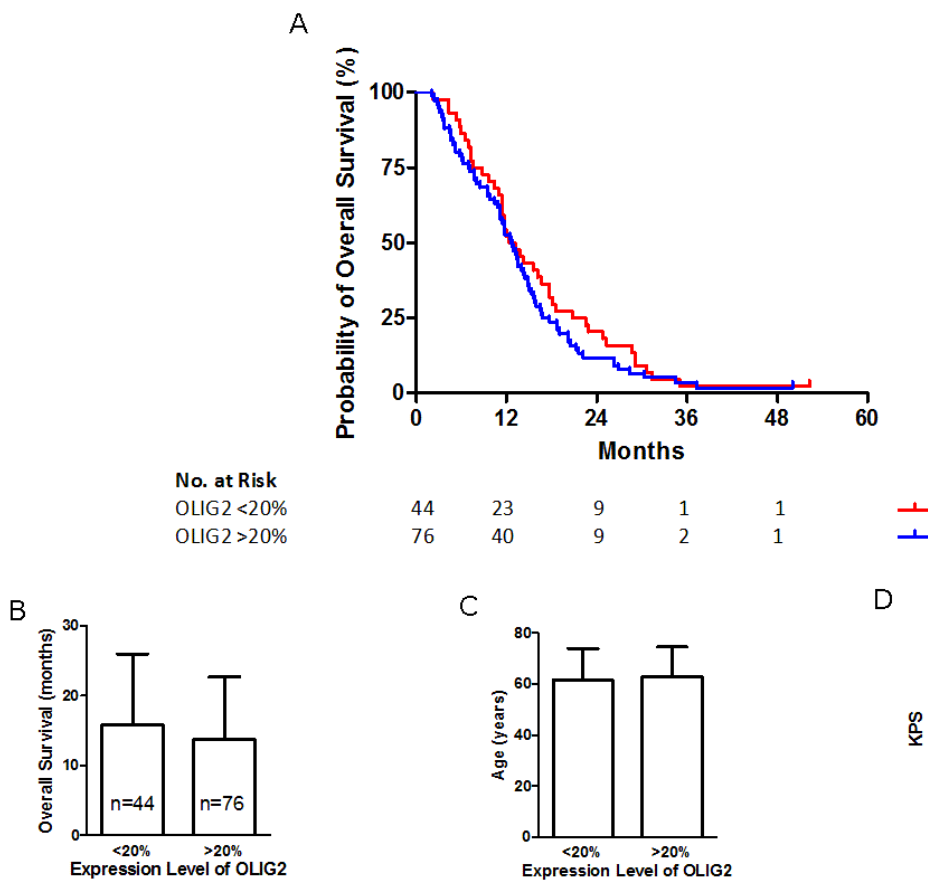

C

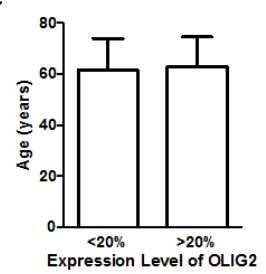

D

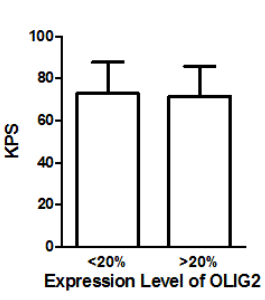

Figure 3.25: Longer mean overall survival of OLIG2 expression below 20\% failed statistical significance. Illustration of overall survival as Kaplan-Meier curve (A) and depiction of overall survival $(\boldsymbol{B})$, age at diagnosis $(\boldsymbol{C})$ and $K P S(\boldsymbol{D})$ as bar graphs. No noticeable difference in age and KPS at the time of diagnosis ( $p=0.6292$ and $p=0.6189$, respectively). Patients with lower immunopositive rates have an advantage regarding overall survival without statistical significance $(p=0.2952)$. Bars represent mean values with standard deviation. Asterisks represent statistically significant differences ( $p$-values $<0.05$ ).

Immunopositivity of over $30 \%$ of the tumor cells was exceeded by 62 samples (see figure 3.26). The remaining 58 samples were slightly younger at the time of diagnosis and held a marginally lower clinical status (61.53 compared to 62.96 years and $70.86 \%$ compared to $73.23 \%$ ). These slight differences in age and KPS were not statistically significant $(\mathrm{p}=0.5179$ and $\mathrm{p}=0.3713$, respectively). Mean overall survival was 16.01 months and therefore almost three months longer than patients with OLIG2 rates over 30\% (13.16 months). The difference in overall survival barely missed statistical significance $(\mathrm{p}=0.0587)$. Multivariate analysis revealed OLIG2 expression below $30 \%$ to be an independent prognostic factor in glioblastoma multiforme $(p=0.0168$, see chapter 3.4). 


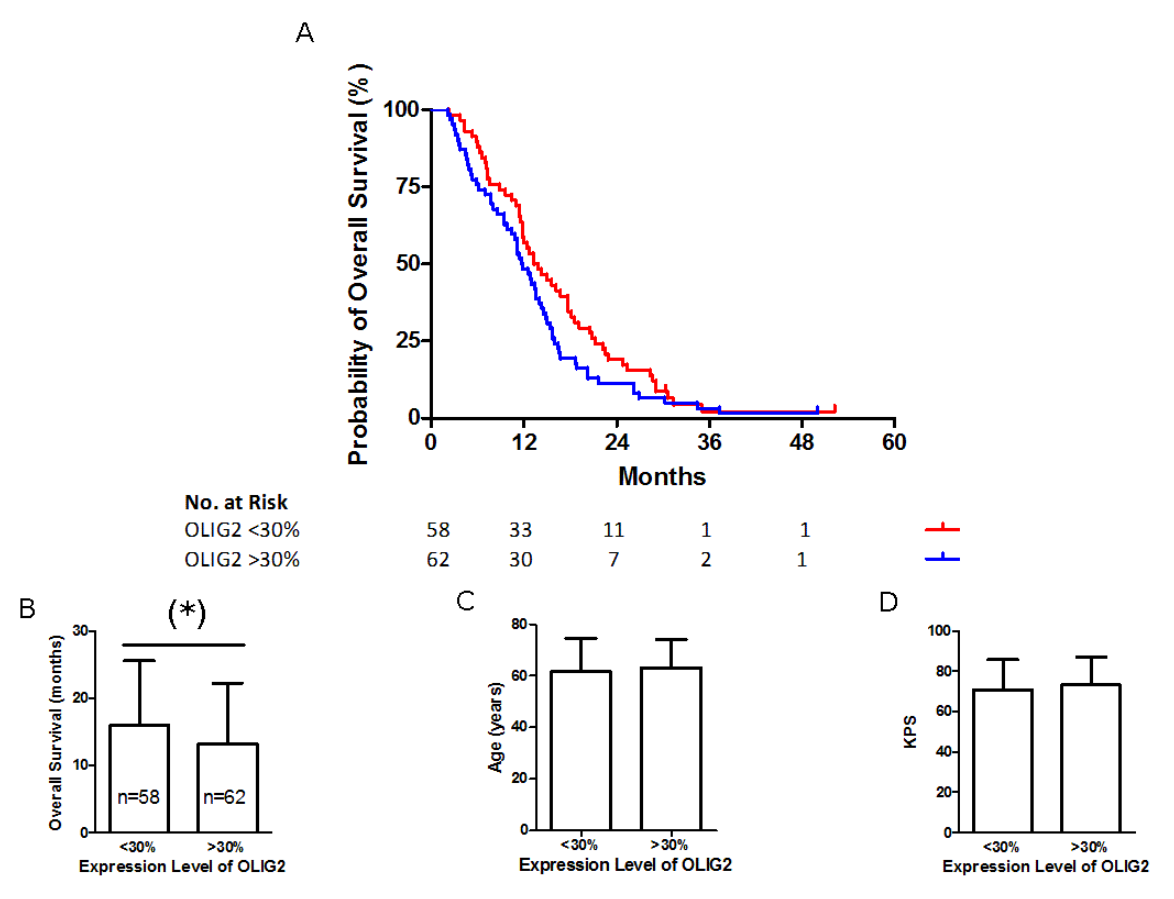

Figure 3.26: OLIG2 expression below 30\% shows a statistical trend towards longer overall survival. Illustration of overall survival as Kaplan-Meier curve (A) and depiction of overall survival $(\boldsymbol{B})$, age at diagnosis $(\boldsymbol{C})$ and $K P S(\boldsymbol{D})$ as bar graphs. There was no considerable difference in age and clinical status at the time of diagnosis ( $p=0.5179$ and $p=0.3713$, respectively). There was a clear trend towards longer overall survival in favor of lower immunopositivity rates, although without statistical significance $(p=0.0587)$. Multivariate analysis revealed samples with OLIG2 below 30\% to have a significantly better prognosis ( $p=0.0168$, see Chapter 3.4). Bars represent mean values with standard deviation. Asterisks represent statistically significant differences (p-values <0.05). Asterisks in parentheses represent significant differences according to multivariate analysis (p-values $<0.05)$.

The majority of GBM samples (76) had an immunopositivity for OLIG2 below $40 \%$ (see figure 3.27). There was no noticeable difference in age when compared to the 44 samples that showed higher expression rates (62.09 and 62.57 years, respectively, $\mathrm{p}=0.828$ ). Like at all other OLIG2 cutoffs, lower immunopositive rates were inclined to have a slightly lower clinical status at the time of diagnosis without statistical significance (71.05\% compared to $73.86 \%, \mathrm{p}=0.2897$ ). A marginal difference in overall survival for lower OLIG2 rates failed to reach statistical significance (14.91 compared to 13.89 months, $\mathrm{p}=0.3087$ ). 


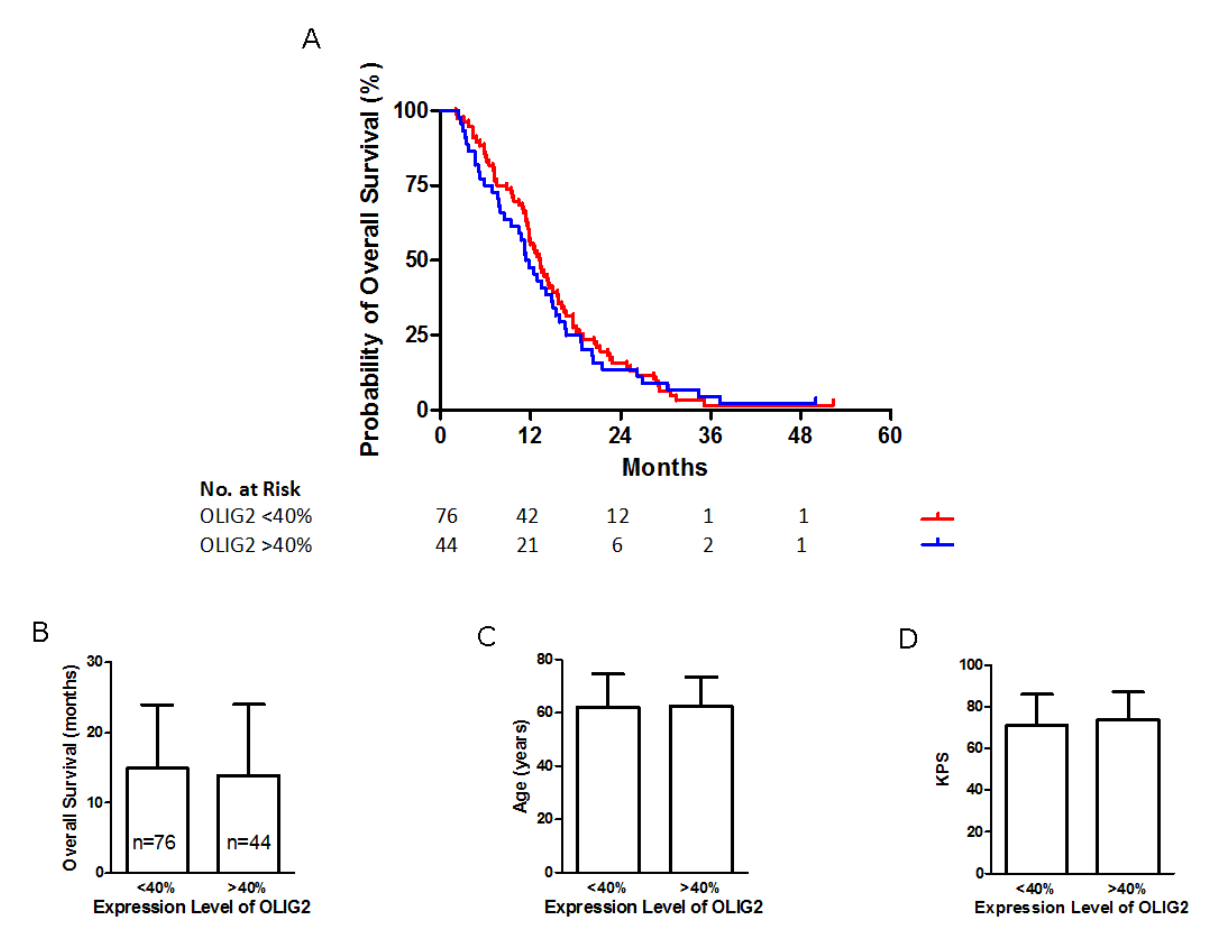

Figure 3.27: OLIG2 cutoff at 40\% without significant differences. Illustration of overall survival as Kaplan-Meier curve (A) and depiction of overall survival (B), age at diagnosis $(C)$ and $K P S(D)$ as bar graphs. There was no significant difference in age at diagnosis $(p=0.828)$, clinical status $(p=0.2897)$ or overall survival $(p=0.3087)$. Bars represent mean values with standard deviation. Asterisks represent statistically significant differences (p-values $<0.05)$.

23 GBM samples were identified to have immunopositive rates of OLIG2 of the majority of tumor cells (over $50 \%$ ). In comparison to the other 97 samples, no considerable difference in age and clinical status at the time of diagnosis was observed (62.42 years and $71.74 \%$ compared to 62.22 years and $72.16 \%$ ). No statistical significance was reached regarding age and KPS $(\mathrm{p}=0.9246$ and $\mathrm{p}=0.8893$, respectively). Similaer to the cutoff at $40 \%$, there was only a slightly longer overall survival without statistical significance for lower immunopositive rates for OLIG2 was seen (14.82 compared to 13.34 months, $\mathrm{p}=0.3259$ ) (see figure 3.28 ). 
A

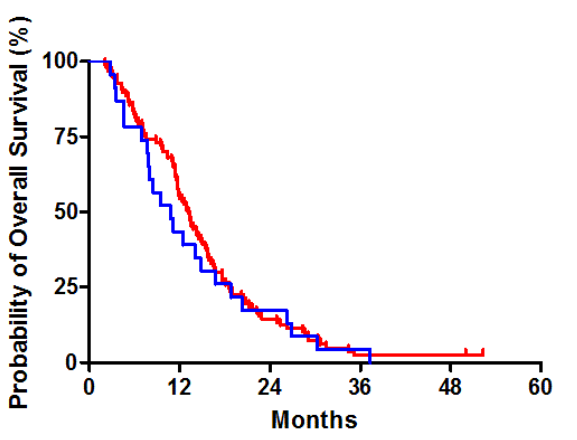

No. at Risk OLIG2 $<50 \%$

$\begin{array}{cccccc}97 & 53 & 14 & 2 & 2 & \text { 土 } \\ 23 & 10 & 4 & 1 & 0 & \text { 土 }\end{array}$

B

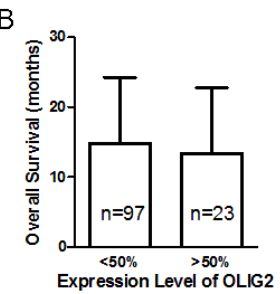

C

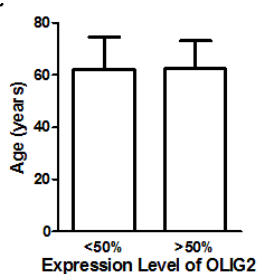

D



Figure 3.28: OLIG2 cutoff at 50\% without significant differences. Illustration of overall survival as Kaplan-Meier curve (A) and depiction of overall survival (B), age at diagnosis $(C)$ and $K P S(D)$ as bar graphs. There was no difference in age $(p=0.9246)$ or clinical status $(p=0.8893)$ at the time of diagnosis as well as overall survial $(p=0.3259)$. Bars represent mean values with standard deviation. Asterisks represent statistically significant differences ( $p$-values $<0.05)$. 


\subsubsection{Ki67}

Ki67 is a widespread tool in pathology laboratories to evaluate the proliferation index of tumor cells. A correlation between Ki67 expression and WHO grading of astrocytomas is well established. Studies that specifically target the prognostic value of Ki67 expression of glioblastomas are rare and also produced contradictory results (see chapter 1.2.7). With a cohort of 120 patients that underwent a similar treatment regimen, this study provides a robust framework to test the prognostic role of Ki67 in glioblastoma multiforme.

After immunohistochemical visualization the proliferation factor Ki67 appears as brown or gray nuclear staining. Among the controls, anaplastic oligodendroglioma (WHO grade III) showed the highest density in immunopositivity (see figure 3.29). Figure 3.30 shows some GBM samples with differing expression rates. About two thirds of the patient collective exhibited 5 to $20 \%$ of their cells as immunopositive (68.33\%), $13.33 \%$ above $20 \%$ and $17.50 \%$ below $5 \%$ (figure 3.31 ). For statistical analysis the sample cohort was divided into two groups according to their Ki67 expression rates. The cutoffs were chosen at 5, 10 and 20\%. According to the exclusion criteria stated in section 2.7 one sample was not included into the statistical analysis of Ki67.



Figure 3.29: Ki67 controls. 400-fold magnification of the controls: cerebellum (A), hippocampus $(\boldsymbol{B})$, cerebral melanoma metastasis $(\boldsymbol{C})$, diffuse oligodendroglioma (WHO grade II) (D) and anaplastic oligodendroglioma (WHO grade III) (E). No image of pilocytic astrocytoma (WHO grade I) control was available. Diffuse oligodendroglioma and melanoma metastasis revealed only scattered single cells with slight immunopositivity. Furthermore cerebellum and hippocampus showed few weak immunopositive cells. Anaplastic oligodendroglioma served as a positive control. 
Identification of Prognostically Relevant Cellular Markers of Differentiation in Glioblastoma

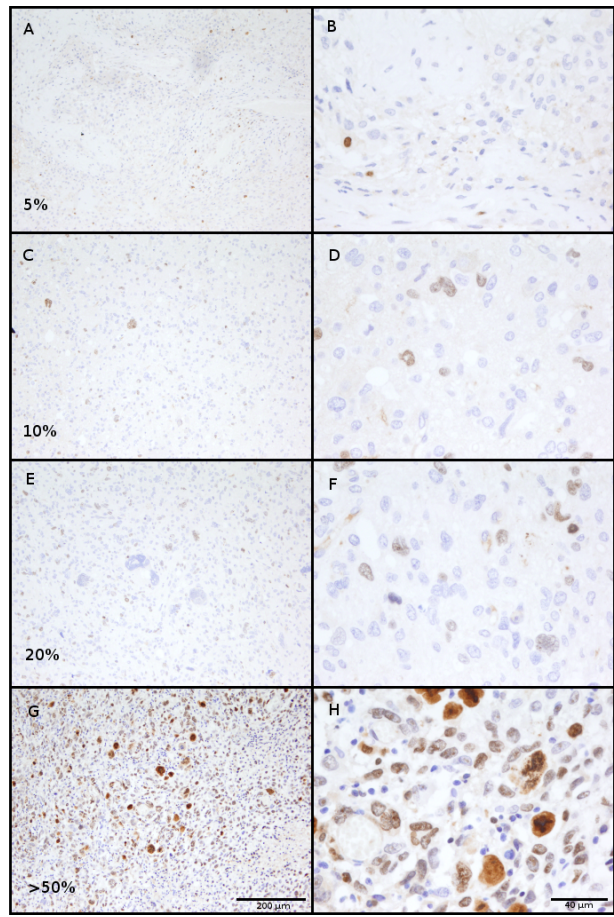

Figure 3.30: Examples of differing Ki67 expression levels. In the upper sample (A and $\boldsymbol{B}$ ) $5 \%$ of the cells are immunopositive. The second and third row feature $10 \%$ ( $C$ and $\boldsymbol{D}$ ) and $20 \%$ ( $\boldsymbol{E}$ and $\boldsymbol{F})$, respectively, while the lowest row shows a sample with a high expression rate of over $50 \%$ ( $\boldsymbol{G}$ and $\boldsymbol{H})$ (100-fold magnification on the left and 400-fold on the right).

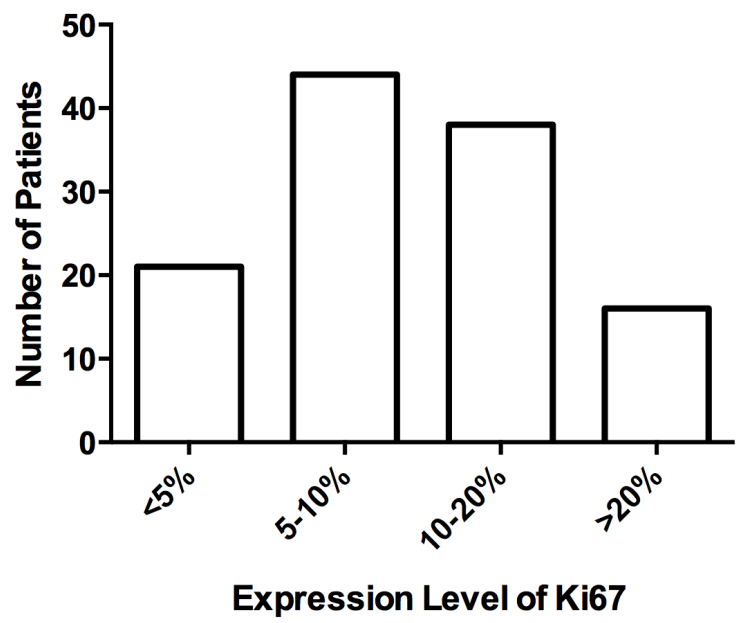

Figure 3.31: Distribution of Ki67 expression rates among GBMs. 
The cutoff at $5 \%$ resulted in 21 samples with a lower expression rate (below 5\%) and 98 GBMs with higher immunopositivity (above 5\%)(see figure 3.32). There was no difference in mean overall survival (below 5\% 14.80 and above 5\% 14.51 months, $\mathrm{p}=0.7457)$. Furthermore, the two groups did not differ in age and clinical status at the time of diagnosis (63.44 years and $72.86 \%$ in comparison to 61.91 years and $71.94 \%$, below and above $5 \%$, respectively) (Age: $\mathrm{p}=0.6339$, KPS: $\mathrm{p}=0.805$ ).

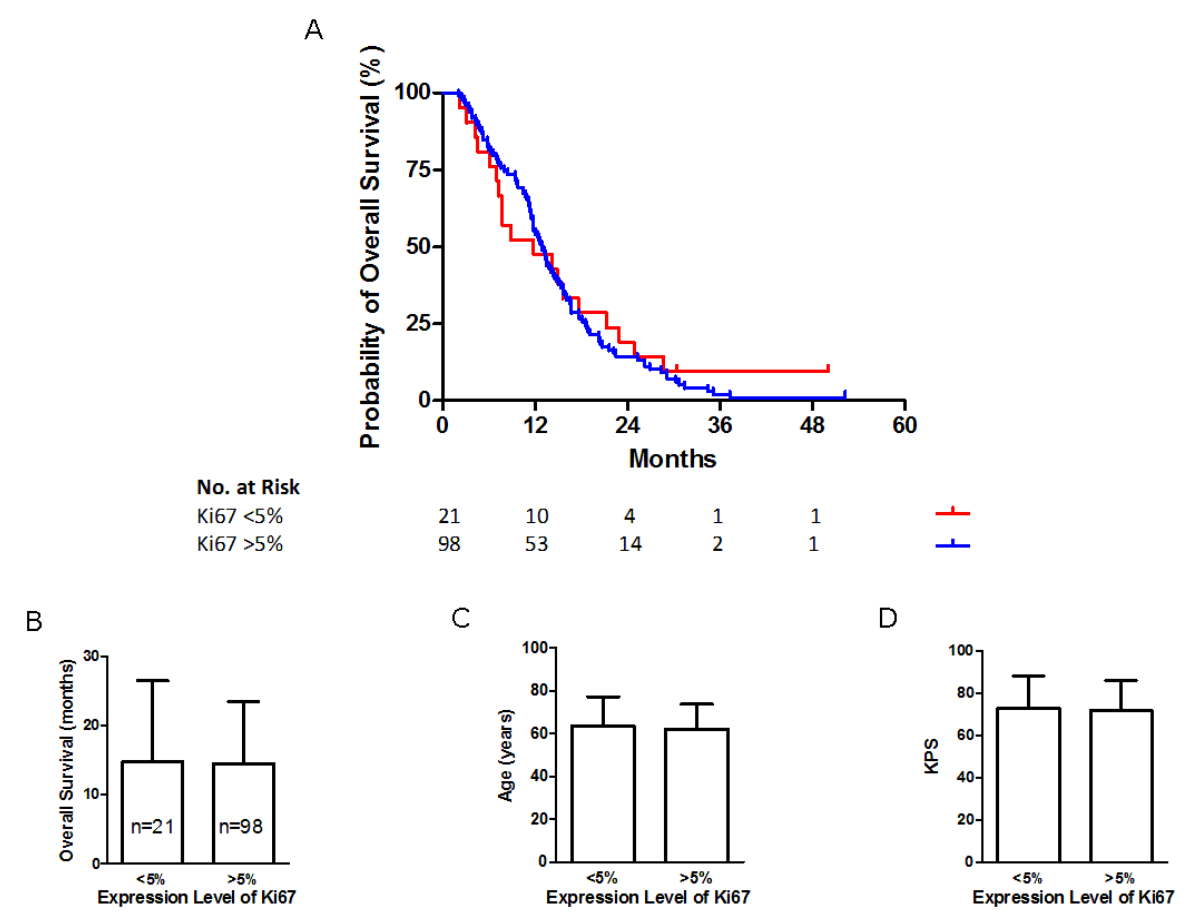

Figure 3.32: Ki67 cutoff at 5\% without significant differences. Illustration of overall survival as Kaplan-Meier curve $(\boldsymbol{A})$ and depiction of overall survival $(\boldsymbol{B})$, age at diagnosis $(\boldsymbol{C})$ and $K P S(\boldsymbol{D})$ as bar graphs. Mean overall survival $(p=0.7457)$, as well as age $(p=0.6339)$ and $K P S(p=0.805)$ at diagnosis were similar among the two subgroups. Bars represent mean values with standard deviation. Asterisks represent statistically significant differences ( $p$-values <0.05).

The next cutoff was chosen at $10 \%$ (see figure 3.33) dividing the patient collective into 65 tumor samples below and 54 samples above that expression rate. Only a slight difference in overall survival was observed without statistical significance (below $10 \%$ 15.03 months and above 10\% 14.00 months, $\mathrm{p}=0.6424)$. Again, age and KPS at diagnosis did not reveal a notable difference (61.27 years and $72.46 \%$ compared to 63.27 years and $71.67 \%$, below and above $10 \%$, respectively) (Age: $\mathrm{p}=0.3531$, KPS: $\mathrm{p}=0.7691$ ). 


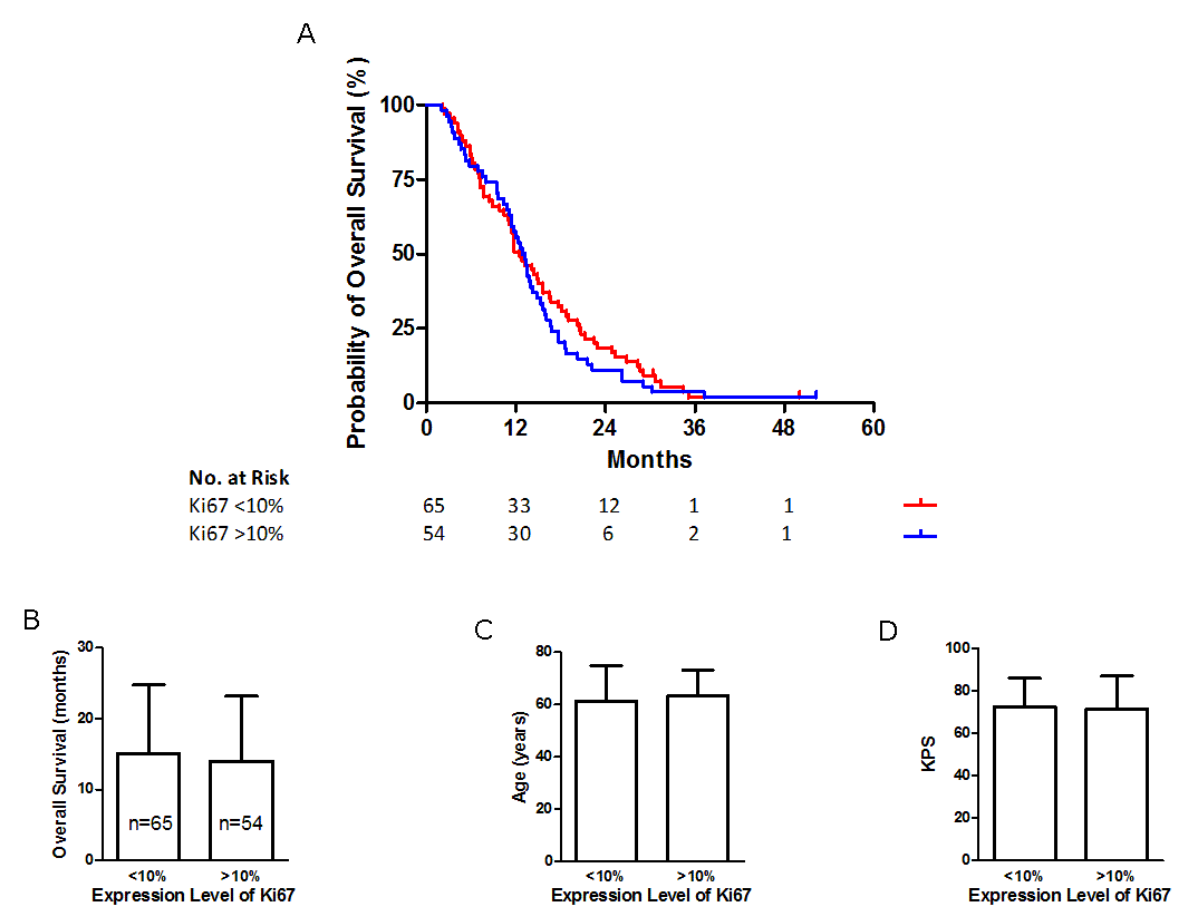

Figure 3.33: Ki67 cutoff at 10\% without significant differences. Illustration of overall survival as Kaplan-Meier curve $(\boldsymbol{A})$ and depiction of overall survival $(\boldsymbol{B})$, age at diagnosis $(\boldsymbol{C})$ and KPS $(\boldsymbol{D})$ as bar graphs. Mean overall survival $(p=0.6424)$, as well as age $(p=0.3531)$ and $K P S(p=0.7691)$ at diagnosis were similar among the two subgroups. Bars represent mean values with standard deviation. Asterisks represent statistically significant differences ( $p$-values $<0.05$ ).

The last cutoff at 20\% split the samples into 103 GBMs with a Ki67 expression rate below $20 \%$ and 16 samples with higher immunopositivity (see figure 3.34). Like the cutoffs at 5 and $10 \%$ there was no apparent difference regarding age and clinical status at diangosis (62.22 years and $72.04 \%$ compared to 61.92 years and $72.50 \%$, below and above $20 \%$ immunopositiviy, respectively). Statistical analysis showed no significant differences (Age: $\mathrm{p}=0.9222$, KPS: $\mathrm{p}=0.9283$ ). Patients exceeding an expression rate of $20 \%$ for Ki67 seemed to have a shorter mean overall survival. This difference failed to reach statistical significance (14.85 months for expression rates below $20 \%$ in contrast to 12.72 months for higher expression rates, $\mathrm{p}=0.2595$ ). 




Figure 3.34: Ki67 cutoff at 20\% without significant differences. Illustration of overall survival as Kaplan-Meier curve $(\boldsymbol{A})$ and depiction of overall survival $(\boldsymbol{B})$, age at diagnosis $(\boldsymbol{C})$ and $K P S(\boldsymbol{D})$ as bar graphs. Age and KPS at diagnosis were without significant differences ( $p=0.9222$ and $p=0.9283$, respectively). Expression rates above 20\% show a shorter mean overall survival but without statistical significance $(p=0.2595)$. Bars represent mean values with standard deviation. Asterisks represent statistically significant differences ( $p$ values $<0.05)$. 


\subsubsection{P53}

The tumor suppressor protein p53 is one of the most important proteins in cancer research and is encoded by the TP53 gene. It can inhibit proliferation after a cell has encountered a certain stress such as DNA damage. Interestingly, several studies showed that TP53 mutations were associated with longer overall survival but mostly due to confounding factors such as younger age and secondary GBM (see chapter 1.2.8). Mutations of TP53 lead to slower protein degradation and subsequent accumulation of the protein.

Immunohistochemical staining highlights nuclei of cells with increased amounts of p53 with brown coloring. Infrequently there were control samples of pilocytic astrocytoma (WHO grade I), melanoma metastasis and diffuse and anaplastic oligodendroglioma (WHO grade II and III, respectively) showing very few scattered positive cells (less than 1\%). Regardless of this observation the controls were viewed as negative. There was no strong positive control (see figure 3.35). The p53 immunopositivity rate varied broadly within the studied GBMs. The majority held $5 \%$ of positive cells or less (see figure 3.36). Figure 3.37 illustrates examples of stained tumor tissue samples with less than $5 \%$ of positive tumor cells, close to $10 \%$ and more than $60 \%$. According to the exclusion criteria (see chapter 2.7) one sample was not considered for statistical analysis. Therefore the immunopositivity rate of p53 and the according clinical impact was studied in 119 instead of 120 GBMs.

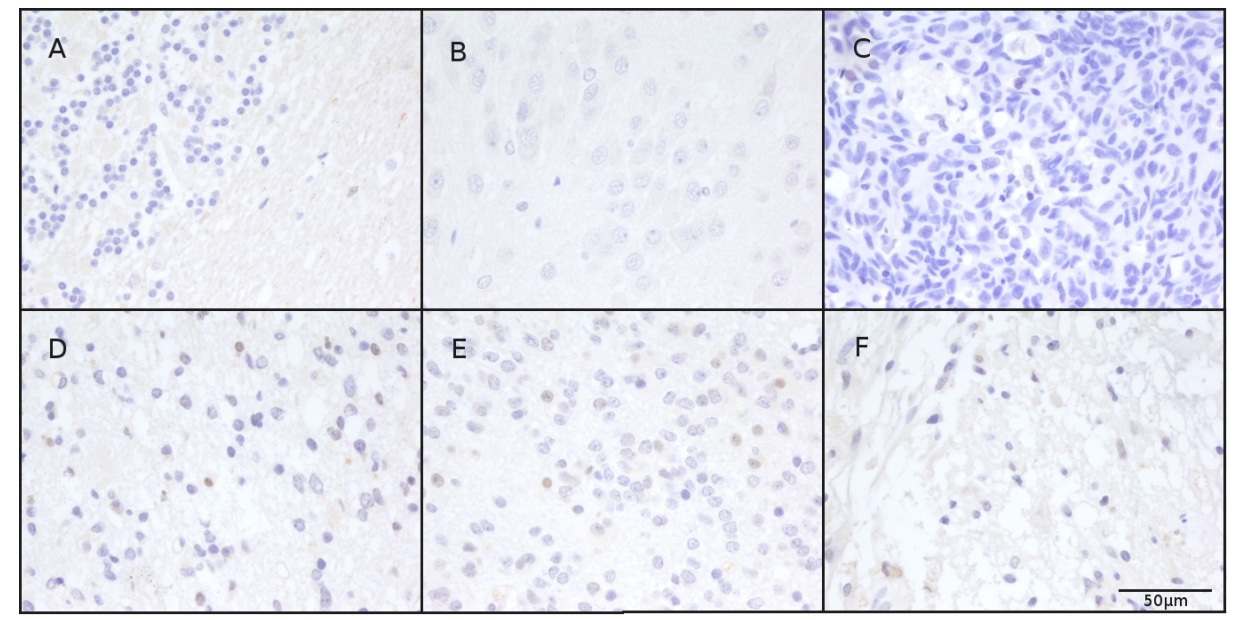

Figure 3.35: P53 controls. 400-fold magnification of the controls: cerebellum (A), hippocampus $(\boldsymbol{B})$, cerebral melanoma metastasis $(\boldsymbol{C})$, diffuse oligodendroglioma (WHO grade II) (D), anaplastic oligodendroglioma (WHO grade III)(E) and pilocytic astrocytoma (WHO grade I) $(\boldsymbol{F})$. All controls were negative for p53 immunohistochemistry. 
Identification of Prognostically Relevant Cellular Markers of Differentiation in Glioblastoma

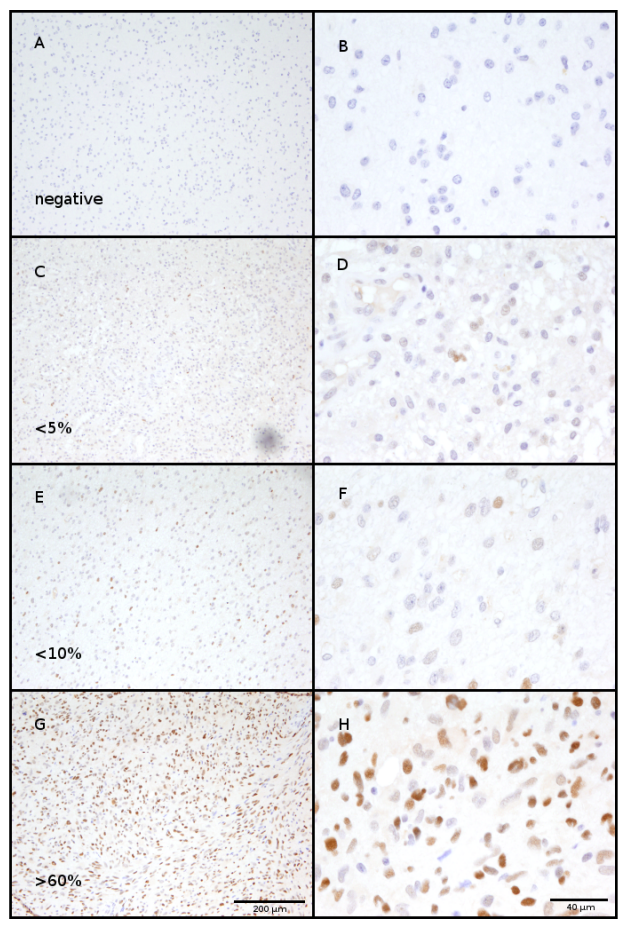

Figure 3.36: Examples of differing p53 immunopositivity rates. The first (A and $\boldsymbol{B})$ row shows a sample without immunopositivity for p53. The second row ( $\boldsymbol{C}$ and $\boldsymbol{D})$ holds less than $5 \%$ of positive cells while the third row (E and $\boldsymbol{F})$ reaches 10\%. The majority of the cells in the sample of the bottom row ( $\boldsymbol{G}$ and $\boldsymbol{H})$ show $p 53$ immunopositivity (100-fold magnification on the left and 400-fold on the right).

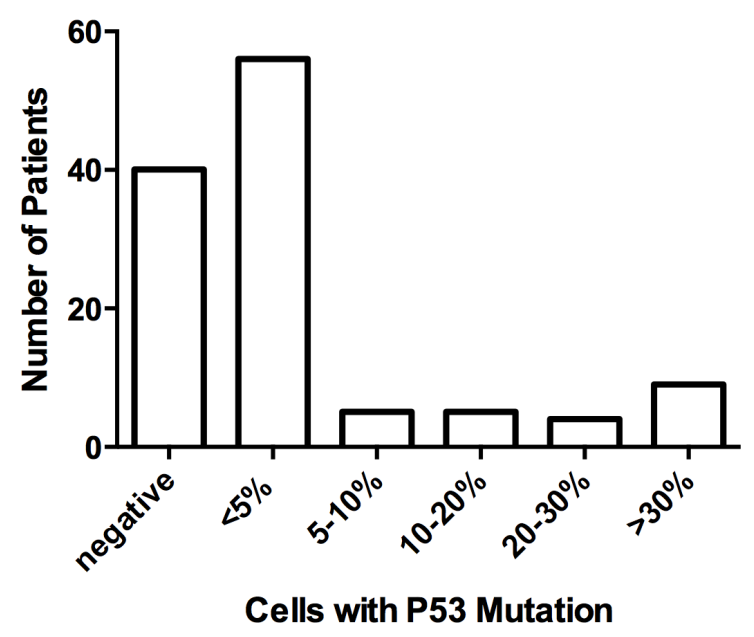

Figure 3.37: Distribution of p53 immunopositivity rates among glioblastomas. 
The first cutoff divided the patient collective into samples with and without p53 positive cells determined by immunohistochemical staining. 40 patients did not show staining for p53 while 79 patients held immunopositivity rates ranging from less than $5 \%$ to over $60 \%$. At the time of diagnosis individuals with p53-positive cells were younger (60.66 years) and had a better clinical status $(74.68 \%)$ than their immunonegative counterparts (65.16 years and 67.75\%) (see figure 3.38). Both differences were statistically significant (Age: $\mathrm{p}=0.04054, \mathrm{KPS}$ : $\mathrm{p}=0.01165$ ). With 15.88 months, immunopositive GBMs also showed longer mean overall survival (compared to 11.95 months). A Kaplan-Meier curve depicts this difference in figure 3.38. Statistical significance was reached $(\mathrm{p}=0.0480)$. Multivariate analysis revealed no statistical significance regarding overall survival $(\mathrm{p}=0.0689$, see section 3.4$)$.

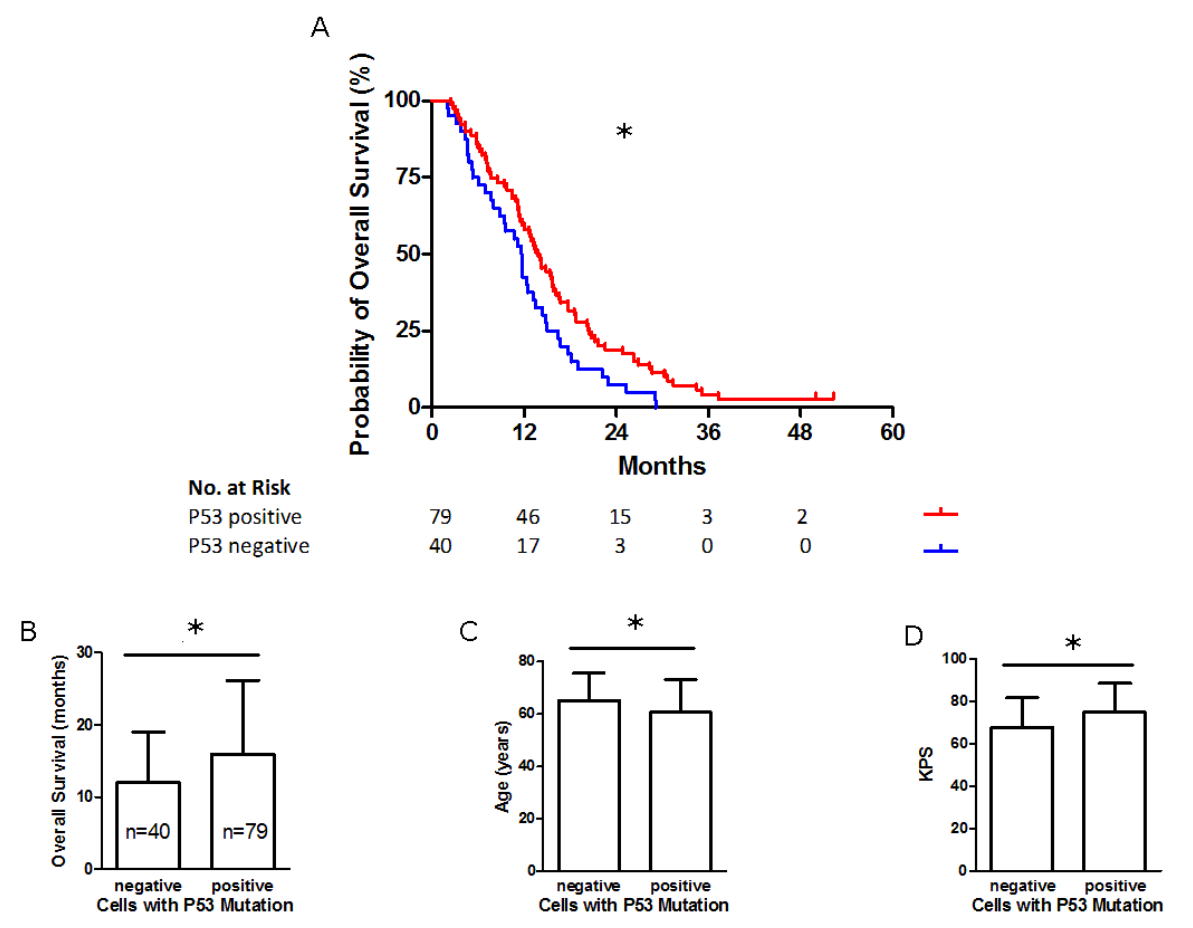

Figure 3.38: GBMs with p53 positive tumor cells have a significantly longer overall survival while being younger and of better clinical status. Illustration of overall survival as Kaplan-Meier curve $(\boldsymbol{A})$ and depiction of overall survival $(\boldsymbol{B})$, age at diagnosis $(\boldsymbol{C})$ and $K P S(\boldsymbol{D})$ as bar graphs. Patients with p53 immunopositivity were younger and held a better clinical status at the time of diagnosis ( $p=0.04054$ and $p=0.01165$, respectively). Furthermore they had a significant advantage regarding overall survival $(p=0.0480)$. However, according to multivariate analysis $p 53$ positivity in general is not an independent prognostic factor $(p=0.0689)$. Bars represent mean values with standard deviation. Asterisks represent statistically significant differences ( $p$-values $<0.05)$. 
A cutoff at $5 \%$ led to 96 cases with p53-positive rates below and 23 above $5 \%$ (see figure 3.39). There was no difference in clinical status between higher and lower immunopositivity rates $(71.30 \%$ and $72.60 \%$, respectively, $\mathrm{p}=0.7365)$ while patients with an increased number of positive cells seemed to be younger at the time of diagnosis (58.85 compared to 62.96 years, $\mathrm{p}=0.1885$ ). There was a slight difference in mean overall survival in favor of p53-positive rates above $5 \%$ without statistical significance (15.53 compared to 14.33 months, $\mathrm{p}=0.9757$ ).

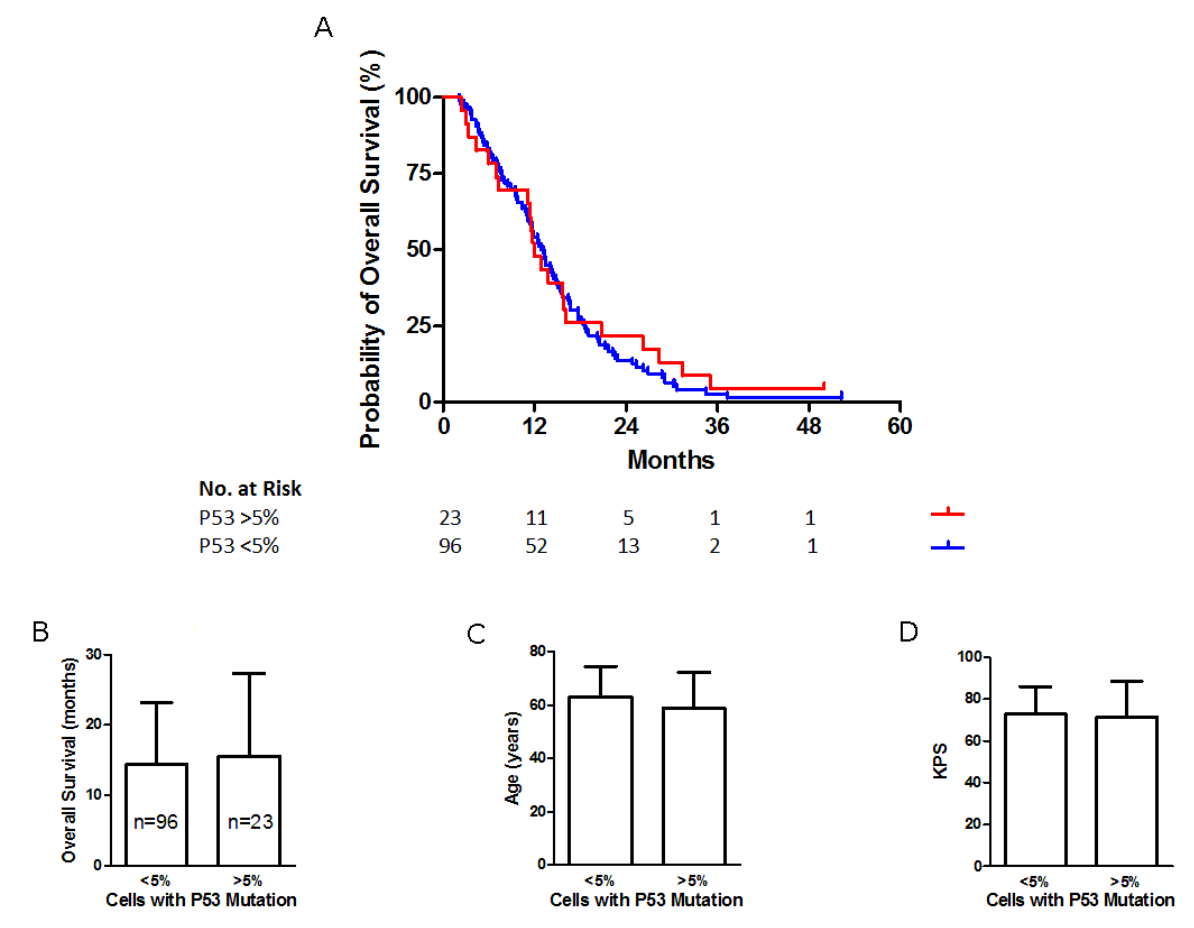

Figure 3.39: P53 cutoff at 5\% without significant differences. Illustration of overall survival as Kaplan-Meier curve $(\boldsymbol{A})$ and depiction of overall survival $(\boldsymbol{B})$, age at diagnosis $(\boldsymbol{C})$ and $K P S(\boldsymbol{D})$ as bar graphs. Patients with increased immunopositivity rates were 4 years younger. This difference was not statistically significant $(p=0.1885)$. KPS was similar in both groups $(p=0.7365)$. There was no significant difference in overall survival $(p=0.9757)$. Bars represent mean values with standard deviation. Asterisks represent statistically significant differences ( $p$-values $<0.05)$.

Only 18 GBMs held an immunopositivity rate of $10 \%$ or higher. This subgroup was approximately 5 and a half years younger at the time of diagnosis (57.42 compared to 63.02 years)(see figure 3.40). The clinical status was merely marginally lower (70.56\% compared to $72.67 \%$ ). Both differences, in age and KPS, failed to reach statistical significance $(\mathrm{p}=0.1215$ and $\mathrm{p}=0.6386$, respectively). Mean overall survival of patients with positivity rates exceeding $10 \%$ was over 2 and a half months longer (16.88 compared to 14.15 months) but without statistical significance $(\mathrm{p}=0.6544)$. 




Figure 3.40: P53 cutoff at 10\% without significant differences. Illustration of overall survival as Kaplan-Meier curve $(\boldsymbol{A})$ and depiction of overall survival $(\boldsymbol{B})$, age at diagnosis $(\boldsymbol{C})$ and $K P S(\boldsymbol{D})$ as bar graphs. With a p53 positive rate above $10 \%$ patients were somewhat younger and held a slightly lower clinical status at the time of diagnosis, however both differences were without statistical significance ( $p=0.1215$ and $p=0.6386$, respectively). Higher positivity rates showed no statistical significance regarding overall survival ( $p=0.6544)$. Bars represent mean values with standard deviation. Asterisks represent statistically significant differences ( $p$-values $<0.05$ ).

Splitting-up the patient collective at an immunopositivity rate of $20 \%$ led to 13 glioblastomas with higher and 106 with lower values. Compared to the cutoff at $10 \%$ the age difference was more pronounced (see figure 3.41). Individuals exceeding $20 \%$ of positive tumor cells were more than 8 years younger at the time of diagnosis $(54.97$ compared to 63.05 years). Even though this difference was not statistically significant $(\mathrm{p}=0.07779)$, there was a trend towards younger age with higher expression rates. Again the clinical status was slightly lower with increased p53 positivity $(70.00 \%$ compared to $72.64 \%)$, but without statistical significance $(\mathrm{p}=0.6624)$. On average individuals with higher immunopositivity rates survived more than three and a half months longer (17.72 compared to 14.17 months). However, this difference failed statistical significance as well $(\mathrm{p}=0.7296)$. 



Figure 3.41: Patients with p53 immunopositivity rates above 20\% showed a tendency towards younger age. Illustration of overall survival as Kaplan-Meier curve (A) and depiction of overall survival $(\boldsymbol{B})$, age at diagnosis $(\boldsymbol{C})$ and $K P S(\boldsymbol{D})$ as bar graphs. Patients with higher positivity rates were over 8 years younger at the time of diagnosis, although failing statistical significance $(p=0.07779)$. There was no statistically significant difference in KPS and overall survival ( $p=0.6624$ and $p=0.7296$, respectively). Bars represent mean values with standard deviation. Asterisks represent statistically significant differences (p-values <0.05).

For further investigations, another cutoff was placed at an immunopositivty rate of $30 \%$ which was only exceeded by 9 GBM samples (see figure 3.42). Compared to the remaining 110 tumors with lower rates of p53 positive cells, these 9 patients were slightly younger at the time of diagnosis (56.41 compared to 62.64 years, $\mathrm{p}=0.2466$ ) and experienced a longer mean overall survival (20.94 compared to 14.04 months). The difference in overall survival was almost seven months but failed statistical significance $(\mathrm{p}=0.3624)$. The clinical status of the two groups was similar $(72.27 \%$ of lower and $73.33 \%$ of higher expression rates, $\mathrm{p}=0.8966$ ). 
A
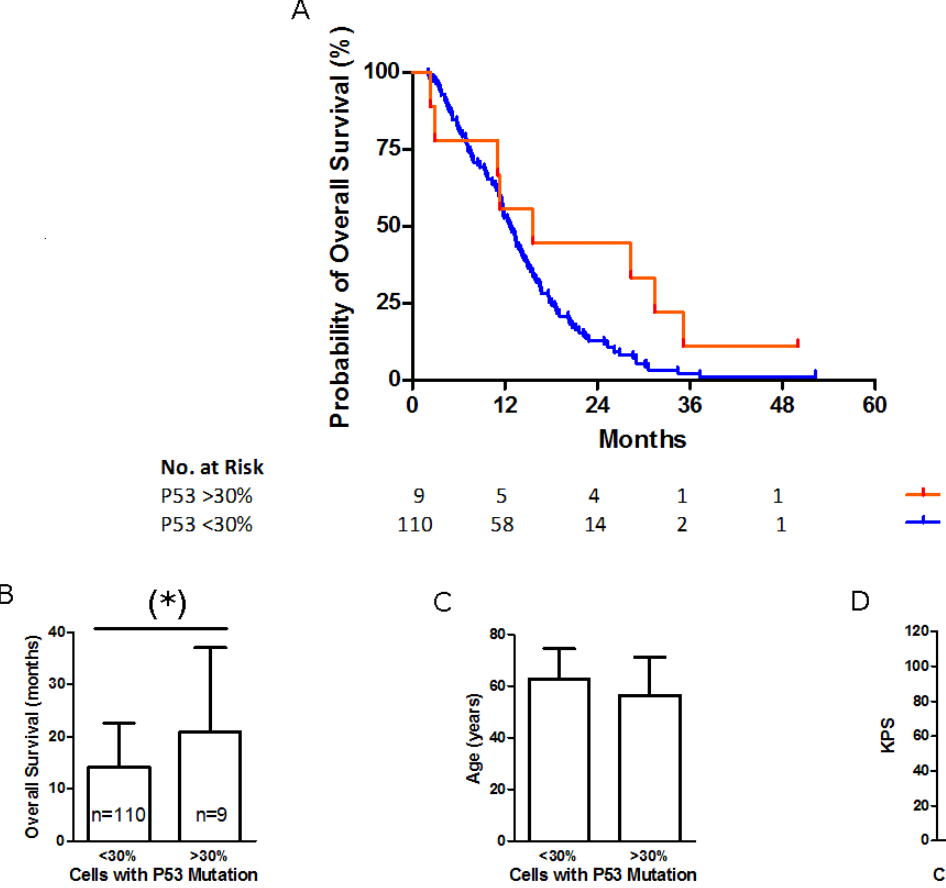

D

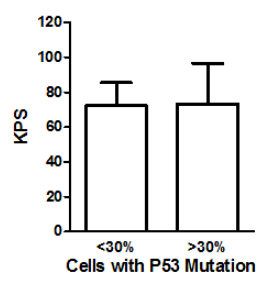

Figure 3.42: Patients with p53 immunopositivity rates above 30\% showed a tendency towards longer overall survival. Illustration of overall survival as Kaplan-Meier curve $(\boldsymbol{A})$ and depiction of overall survival $(\boldsymbol{B})$, age at diagnosis $(\boldsymbol{C})$ and $K P S(\boldsymbol{D})$ as bar graphs. No difference in KPS was observed $(p=0.8966)$ while patients with increased rates of p53 positive cells were over 6 years younger and lived almost seven months longer. No statistical significance regarding longer overall survival $(p=0.3624)$ and age at diagnosis $(p=0.2466)$ was reached. Bars represent mean values with standard deviation. Asterisks represent statistically significant differences ( $p$-values $<0.05)$. 


\subsubsection{GFAP}

Glial fibrillary acidic protein (GFAP) plays an important role in brain development and the recovery of the central nervous system from conditions such as trauma or inflammation. Astrocytic tumors are known to hold a high expression rate of this intermediate filament. Serum levels were shown to correlate with tumor volume and WHO grading while data on its prognostic role is scarce (see chapter 1.2.9).

In this study all GBM tumor tissue samples showed some degree of GFAP expression resulting in perinuclear brown coloring. There was no immunonegative sample. For statistical purposes immunopositivity was quantified with the help of a scoring system (see figure 3.44). Only a few samples showed staining of merely single scattered cells and received a score of $+/$-. If there were many immunopositive tumor cells while the majority of the sample remained immunonegative, a score of + was given. Tissue samples with the majority of cells showing perinuclear staining or a homogeneous coloring of the cytoplasm throughout the sample were given the highest scoring of ++ . In some cases a tissue cylinder held areas of strong $(++)$ and weak $(+)$ immunostaining resulting in the score $+/++$. The white matter of the cerebellum, diffuse and anaplastic oligodendroglioma (WHO grade II and III, respectively) and pilocytic astrocytoma (WHO grade I) served as strong positive controls (see figure 3.43). Cutoffs were chosen at,$++/++$ and ++ in order to divide the patient collective into subgroups of differing expression levels.

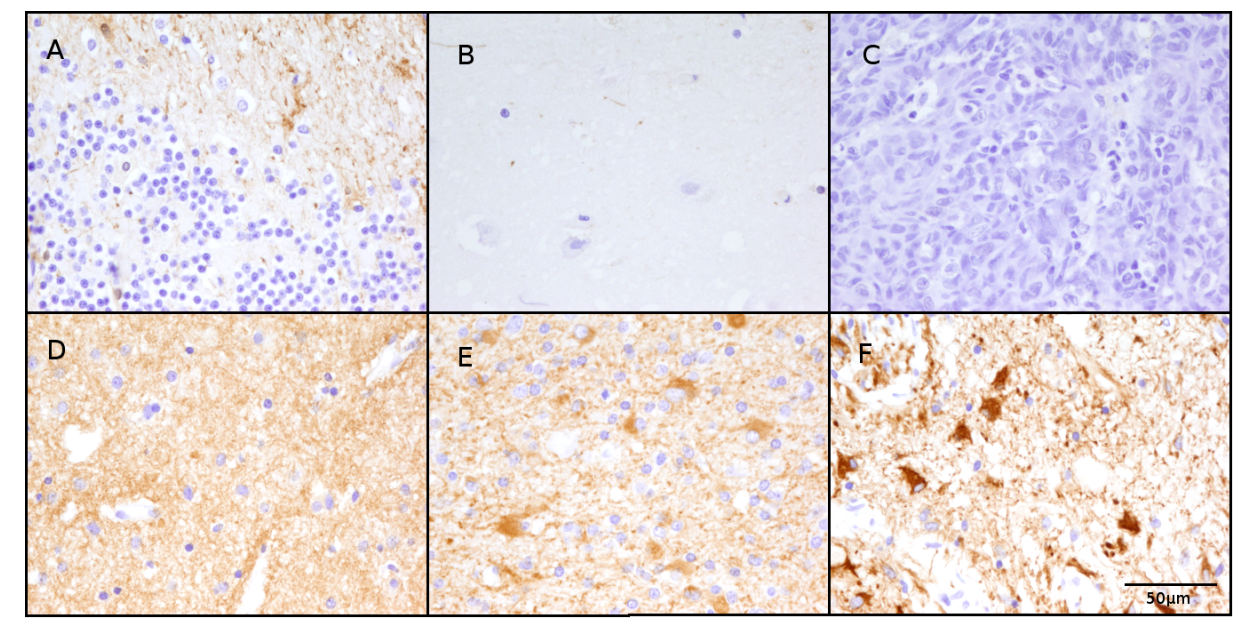

Figure 3.43: GFAP controls. 400-fold magnification of the controls: cerebellum (A), hippocampus $(\boldsymbol{B})$, cerebral melanoma metastasis $(\boldsymbol{C})$, diffuse oligodendroglioma (WHO grade II) (D), anaplastic oligodendroglioma (WHO grade III) (E) and pilocytic astrocytoma (WHO grade I) (F). The white matter of the cerebellum as well as diffuse and anaplastic oligodendrogliomas and pilocytic astrocytoma served as stong positive controls. 


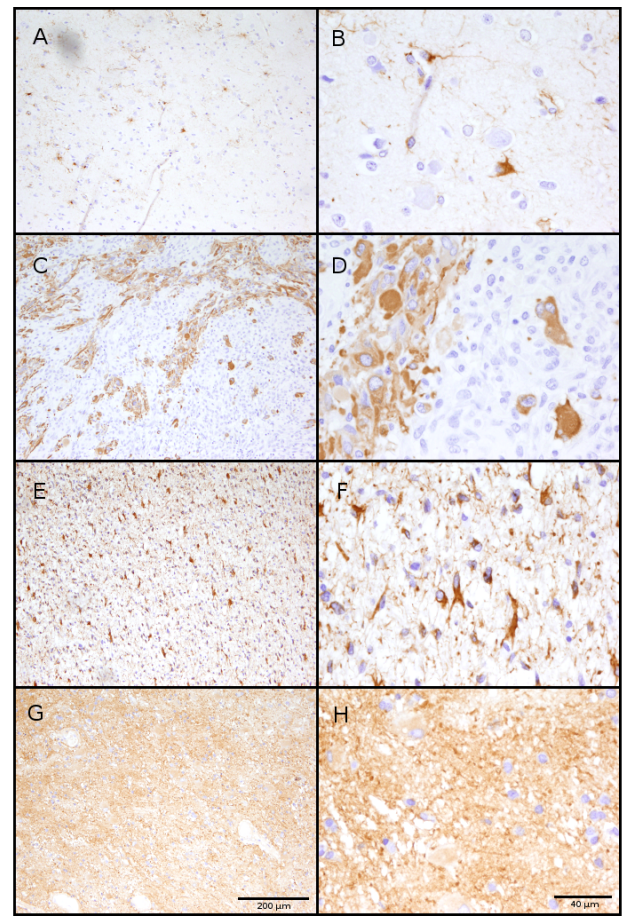

Figure 3.44: Quantification of $\boldsymbol{G F A P}$ immunopositivity. $\boldsymbol{A}$ and $\boldsymbol{B}$ show a tumor tissue sample with many interspersed immunopositive cells while the majority of the cells remains unstained $(+)$. Samples with a majority of immunopositive cells ( $\boldsymbol{E}$ and $\boldsymbol{F}$ ) or a homogeneous perinuclear coloring $(\boldsymbol{G}$ and $\boldsymbol{H})$ were both provided with a score of ++ . Some samples had areas of strong and weak immunostaining, thus receiving a score of $+1++(C$ and D). (100-fold magnification on the left and 400-fold on the right).

The first cutoff divided the patient collective into 14 samples with a score of + or lower and a group of 116 samples with higher expression values. With 54.99 years, tumor samples with lower expression levels were associated with younger age at the time of diagnosis than samples with higher GFAP expression levels (63.23 years). This difference failed to reach statistical significance $(\mathrm{p}=0.07887)$. On the other hand, there was no considerable difference in clinical status at the time of diagnosis $(71.43 \%$ and $72.17 \%$ for lower and higher expression levels, respectively, $\mathrm{p}=0.9028$ ). Likewise, mean overall survival did not differ between the two groups (14.30 months for lower and 14.57 months for higher expression levels, $\mathrm{p}=0.3894$ ) (see figure 3.45). 


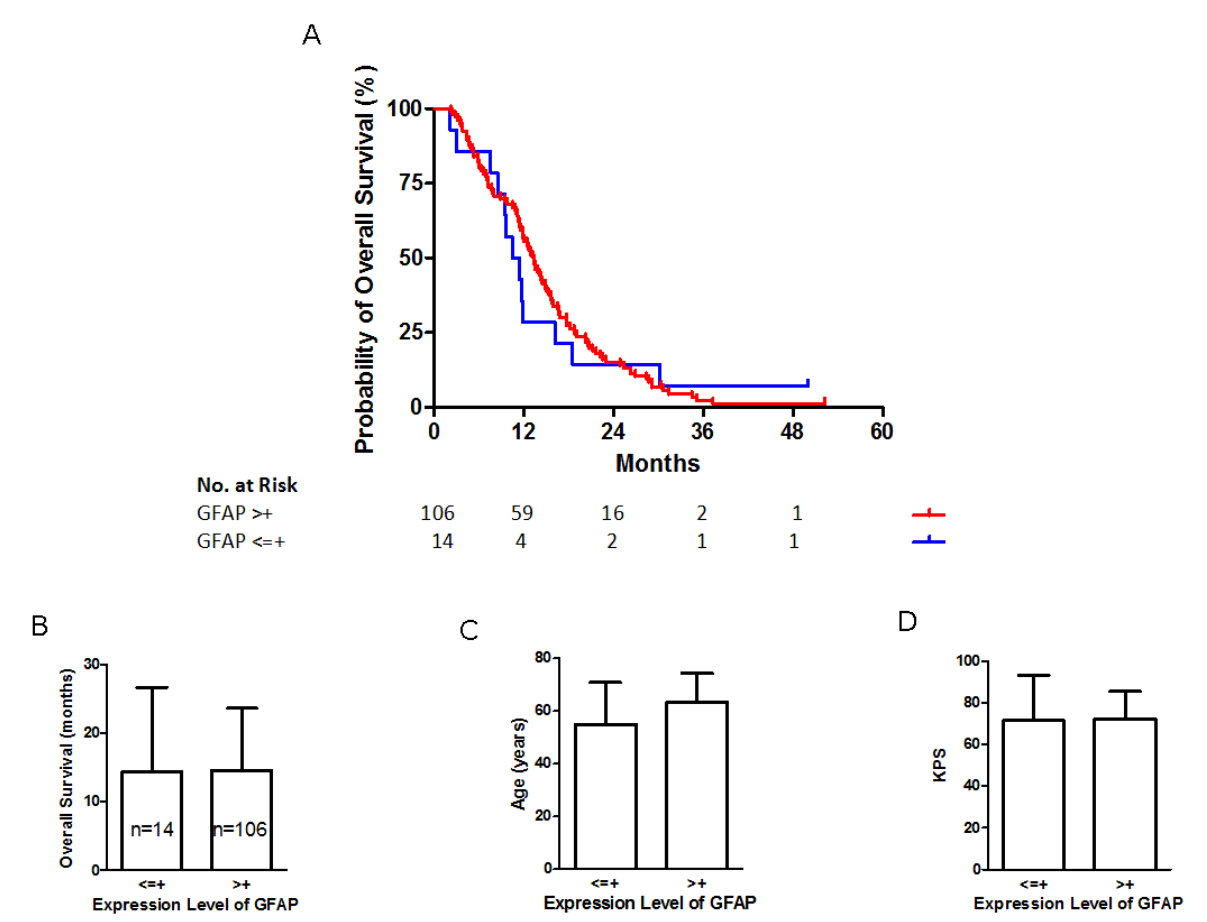

Figure 3.45: A GFAP score below + showed a tendency towards younger age. Illustration of overall survival as Kaplan-Meier curve $(\boldsymbol{A})$ and depiction of overall survival $(\boldsymbol{B})$, age at diagnosis $(\boldsymbol{C})$ and KPS $(\boldsymbol{D})$ as bar graphs. The subgroup with GFAP expression levels below + showed a younger age at the time of diagnosis, however, without statistical significance $(p=0.07887)$. Clinical status and overall survival were similar in both groups ( $p=0.9028$ and $p=0.3894$, respectively). Bars represent mean values with standard deviation. Asterisks represent statistically significant differences ( $p$-values $<0.05)$.

The next cutoff was set at $+/++$. This divided the samples into 48 equal or below and 72 above the expression value of $+/++$. Mean overall survival did not show a notable distinction between the two groups (below/equal $+/++14.40$ months, above $+/++14.63$ months, $\mathrm{p}=0.9447)$. Individuals with an expression level of GFAP above $+/++$ were slightly older and held a minimally better clinical status at the time of diagnosis (63.56 years and $73.06 \%$ in comparison to 60.32 years and $70.63 \%$ ). The small differences in age and KPS were without statistical significance $(\mathrm{p}=0.1474$ and $\mathrm{p}=0.3745$, respectiveley) (see figure 3.46). 
A
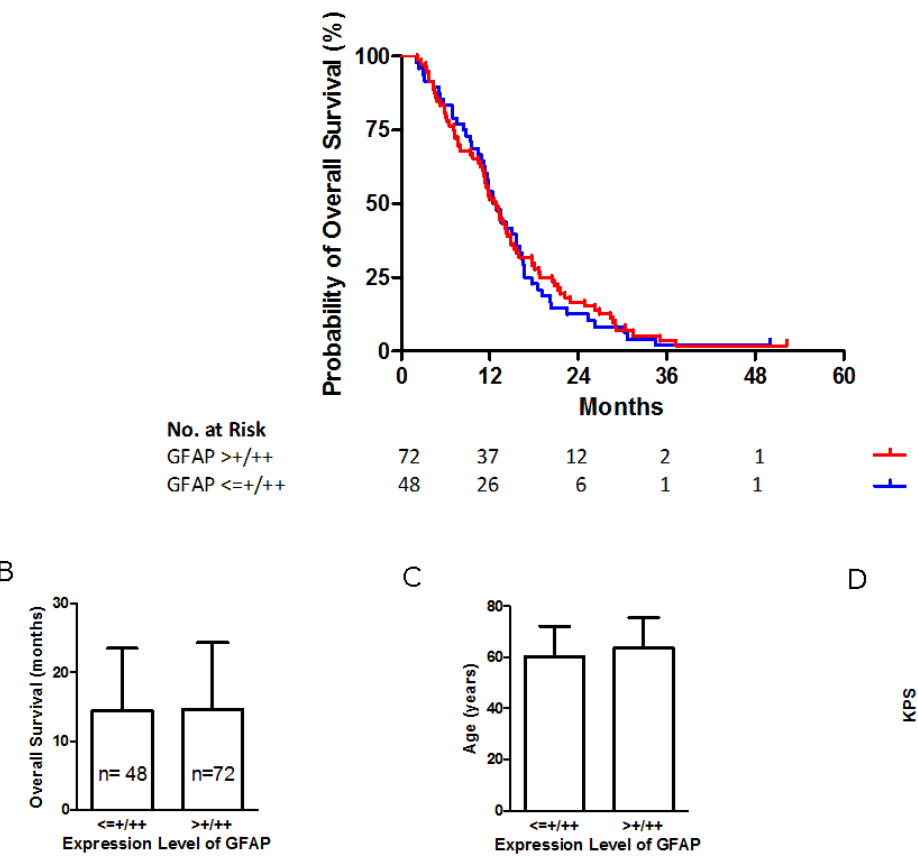

Figure 3.46: GFAP cutoff at $+/++$ without significant differences. Illustration of overall survival as Kaplan-Meier curve $(\boldsymbol{A})$ and depiction of overall survival (B), age at diagnosis $(\boldsymbol{C})$ and KPS $(\boldsymbol{D})$ as bar graphs. Age and KPS at the time of diagnosis as well as overall survival did not show a statistical difference $(p=0.1474, p=0.3745$ and $p=0.9447$, respectively). Bars represent mean values with standard deviation. Asterisks represent statistically significant differences ( $p$-values $<0.05)$.

A final cutoff was set at ++ to separate the 44 samples that scored the maximum GFAP expression from the 76 other samples that showed a lower scoring. As seen in figure 3.47 there was no striking difference in mean overall survival (below ++ 14.28 months, ++14.97 months, $\mathrm{p}=0.9567$ ), KPS (below $++72.24 \%,++71.82 \%$, $\mathrm{p}=0.8789$ ) or age at diagnosis (below ++62.26 months, ++62.27 months, $\mathrm{p}=0.9992$ ). 




B

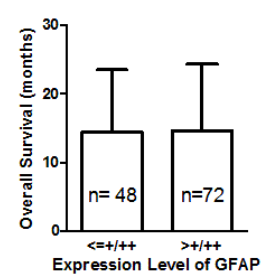

C

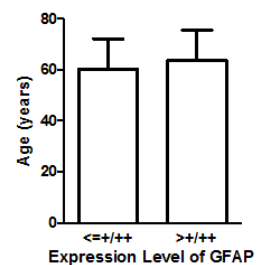

D

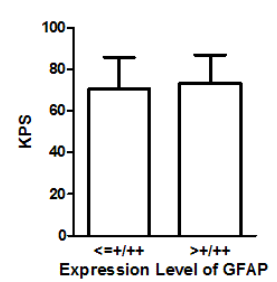

Figure 3.4\%: GFAP cutoff at ++ without significant differences. Illustration of overall survival as Kaplan-Meier curve $(\boldsymbol{A})$ and depiction of overall survival $(\boldsymbol{B})$, age at diagnosis $(\boldsymbol{C})$ and $K P S(\boldsymbol{D})$ as bar graphs. There were no notable differences among the two subgroups regarding mean overall survival $(p=0.9567)$, age $(p=0.9992)$ and KPS $(p=0.8789)$. Bars represent mean values with standard deviation. Asterisks represent statistically significant differences ( $p$-values <0.05). 


\subsection{Multivariate Analysis}

The multivariate analysis was performed with the Cox regression model. In the previous Kaplan-Meier analyses age and the clinical status at the time of diagnosis as well as TP53 mutation in general were identified as significantly associated with a difference in overall survival. In regard to other prognostic markers multivariate analysis was applied to reveal independent prognostic impacts. Together with the above mentioned factors, other markers were included that showed a statistical trend in the Kaplan-Meier analysis (EOR and OLIG2>30\%) as well as IDH-1 mutation, due to its well established prognostic role. MVA showed no significant impact of age at the time of diagnosis. On the other hand, better clinical status (KPS) and OLIG2 expression levels smaller than $30 \%$ led to significantly longer overall survival (see table 3.1). Thus these three parameters are considered independent prognostic factors. Gross total resection showed a trend towards longer overall survival compared to partial resection and biopsy. TP53 mutation turned out not to be an independent prognostic factor but it showed a mentionable statistical trend. IDH-1 mutation did neither show statistical significance nor a trend. Due to incomplete data, two cases could not be included in the multivariate analysis.

Table 3.1: Results of the Cox regression analysis.

\begin{tabular}{|l|l|l|l|l|l|}
\hline Variable & Coefficient & Standard error & Hazard ratio & $95 \%$ CI & p-value \\
\hline Age & 0.008021 & 0.009636 & 1.008053 & $0.9892-1.0273$ & 0.40516 \\
KPS & -0.022345 & 0.007100 & 0.977903 & $0.9644-0.9916$ & $0.00165 * *$ \\
EOR & 0.429495 & 0.228435 & 1.536482 & $0.9819-2.4042$ & 0.06009. \\
IDH-1mt & -0.016053 & 0.504854 & 0.984075 & $0.3658-2.6470$ & 0.97463 \\
p53mt & -0.391844 & 0.215431 & 0.675810 & $0.4430-1.0309$ & 0.06893. \\
Olig2 $>30 \%$ & 0.471985 & 0.197427 & 1.603173 & $1.0888-2.3606$ & $0.01682 *$ \\
\hline
\end{tabular}

p-values:.$<0.1 ;^{*}<0.05 ; * *<0.01$ 


\section{Chapter 4}

\section{Discussion}

\subsection{The Patient Collective}

With a mean overall survival of 14.6 months glioblastoma multiforme is the deadliest and unfortunately the most common primary brain tumor (Stupp et al. 2005). About 3-5\% exceed an overall survival of 36 months (Krex et al. 2007). This malignancy mainly afflicts the elderly with an average age of 64 years while almost any age group can develop the disease. It is known to have a predominance among males (male to female ratio 1.56) (Dolecek et al. 2012). The patient collective that was investigated in this study showed a mean overall survival of 14.54 months, while $2.5 \%$ lived longer than 36 months. On average the age and KPS at the time of diagnosis were 62.26 years and $72.08 \%$. The male to female ratio was 1.50 . Thus the sample collective subject to this study held clinical features comparable to the findings in the current literature. However, this study only included patients who received adjuvant therapy with alkylating chemotherapeutic agents and radiation therapy. Patients receiving adjuvant monotherapy were not considered. Furthermore, some patients who only received tumor biopsy and no further surgical resection were not included due to the small tumor samples that were not suitable for TMA processing. 


\subsection{The Prognostic Impact of Clinical Factors}

\subsubsection{Sex}

The prognostic role of biological gender has been addressed in many clinical studies, yet contradictory results have been published. Several authors suggested an advantage of females (Caloglu et al. 2009, Johnson et al. 2012) while others showed no prognostic effect of sex (Scott et al. 1998) or indicated longer overall survival for male patients (Habberstad et al. 2012). Verger et al., on the other hand, suggested that female patients tended to have a lower clinical status and more advanced age at the time of diagnosis when compared to their male counterparts. Therefore treatment of glioblastoma multiforme in women was more inclined towards biopsy and adjuvant monotherapy than gross total resection and adjuvant radiochemotherapy resulting in the tendency for shorter mean overall survival. However, multivariate analysis proved that gender itself was not an independent prognostic factor (Verger et al. 2011).

The data obtained in this study did show a shorter mean overall survival of women (13.74 months compared 15.07 months) and a lower clinical status at the time of diagnosis $(70.21 \%$ compared to $73.33 \%$ ) while both differences failed to reach statistical significance $(\mathrm{p}=0.2456$ and $\mathrm{p}=0.2552$, respectively). There was no considerable difference in age (62.12 years in women and 62.36 years in men, $\mathrm{p}=0.9138$ ) or inclination towards less radical surgical resection $(77.08 \%$ of women and $77.78 \%$ of men received gross total resection). Out of 10 patients with an overall survival of less than 6 months 7 were male. 18 patients exceeded an overall survival of 24 months of which 12 were male. This fits to the male to female ratio of the patient collective indicating that the extreme overall survival times are evenly distributed among both sexes. Based on the results obtained, sex as an independent factor is unlikely to have an impact on overall survival in glioblastoma.

\subsubsection{Age and KPS}

Age and functional status (KPS) at the time of diagnosis of glioblastoma are well established prognostic factors (Lacroix et al. 2001, Laws et al. 2003, Habberstad et al 2012). Our data showed a significant survival advantage of patients below 60, 65 and 70 years of age $(\mathrm{p}=0.0234, \mathrm{p}=0.0010$ and $\mathrm{p}=0.0278$, respectively) and a non-significant trend towards longer overall survival of individuals younger than 55 years $(\mathrm{p}=0.0815)$, thus confirming the prognostic effect of age in GBM. 
Karnofsky Performance Score showed significant correlation with overall survival $(\mathrm{p}=0.0006)$. Cutoffs were placed at 60,70,80 and $90 \%$ and all showed considerable longer survival rates for the higher scores. However, only the cutoffs at 80 and $90 \%$ were statistically significant ( $\mathrm{p}=0.0020$ and $\mathrm{p}=0.0278$, respectively) while 60 and $70 \%$ merely displayed a statistical trend $(\mathrm{p}=0.0825$ and $\mathrm{p}=0.0539$, respectively). Many neurooncological centers offer adjuvant treatment depending on the postoperative funcional status of the patient. A KPS of at least $70 \%$ is widely accepted as the threshold for adjuvant chemotherapy due to its highly prognostic value. However, in this case statistical significance was not reached for the cutoff at $70 \%(\mathrm{p}=0.0539)$. This study was designed to include tumors that received adjuvant radiotherapy and chemotherapy. Only 17 of the 120 patients held a KPS below 70\%. Patients who received adjuvant monotherapy or no further treatment at all due to poor clinical status were not included. Therefore a bias towards better overall KPS of the patient collective was to be expected. But since the main objective of this study is the prognostic value of immunohistochemical markers, it is more suitable to have a patient collective who received similar treatment.

Surprisingly, multivariate analysis with the help of Cox regression hazard analysis showed no statistical significance for age $(\mathrm{p}=0.4052)$ while clinical status (KPS) was revealed to be a highly significant independent prognostic factor $(p=0.00165$, see section 3.4).

\subsubsection{Extent of Resection}

Several studies regarding the extent of tumor resection and its prognostic value in glioblastoma have been published (Yong and Lonser 2011, Sanai et al. 2011, Chandana et al. 2008, Sanai and Berger 2008, Lacroix et al. 2001, Simpson et al. 1993). The results obtained in this study did not show a significantly prolonged overall survival for patients who received gross total resection in the Kaplan-Meier survival analysis ( $p=0.1583)$, even though patients with extensively resected lesions reached a longer mean overall survival (15.26 compared to 12.03 months). Cox regression analysis revealed that gross total resection showed a strong trend towards being an independent prognostic factor $(\mathrm{p}=0.0601$, see section 3.4$)$.

However, in this study no detailed data regarding the percentage of tumor resection was gathered. The patient collective was divided into a group that underwent gross total resection and a group that was partially resected or biopsied according to the surgical reports. Therefore a comparison with the established data in the literature and further discussion is difficult. Nevertheless, individuals who underwent gross total resection were 4 years older than their partially resected or biopsied counterparts (63.15 compared to 59.21 years). This difference was not significant $(\mathrm{p}=0.1764)$. 


\subsubsection{Chemotherapy}

Chemotherapy with alkylating agents has been in use for the adjuvant treatment of glioblastoma for many years. The contemporary standard chemotherapy is done with temozolomide concomitantly with external beam radiation therapy (Stupp et al. 2005). The majority of the patient collective received TMZ (97 of 120 patients). 15 were treated before the Stupp-era and were given ACNU. They showed similar mean overall survival as well as age and KPS. Interestingly, 8 patients that received local BCNU in form of a Gliadel ${ }^{\circledR}$ wafer in the resection cavity, in addition to systemic TMZ, showed a prolonged overall survival of 20.29 months, compared to 14.06 months for individuals that received sole TMZ chemotherapy. Although not significant, a statistical trend towards longer overall survival of patients that received BCNU in addition to TMZ was observed $(\mathrm{p}=0.0958)$. Furthermore, the 8 cases showed a trend towards higher KPS $(\mathrm{p}=0.07665)$ at the time of diagnosis $(77.50 \%$ compared to $71.86 \%$ ). They were also younger (55.63 years compared to 63.18 years), however without reaching statistical significance $(\mathrm{p}=0.1172)$.

The statistical trend towards better clinical status and the non-significantly younger age may present a possible selection bias. Consequently the trend towards longer overall survival may be due to the two confounding prognostic factors, age and KPS, even though both variables were not significantly different when compared to the cases that received TMZ. It is possible, that the additional local chemotherapy was administered more often to younger patients with better clinical status. Even though several studies showed the effectiveness of Gliadel ${ }^{\circledR}$ wafers regarding prolonged overall survival in GBM (Hart et al. 2011, Westphal et al. 2006), the effectiveness of the adjuvant use additionally to TMZ is still unclear (Catalan-Uribarrena et al. 2012, Bock et al. 2010, McGirt et al. 2009a). The data presented do not show an advantage in overall survival. However, only 8 cases were assessed. 


\subsection{Prognostic Role of Immunohistochemical Markers}

\subsubsection{IDH-1 Mutation}

Since 2013 IDH-1 mutation is proclaimed as a reliable marker for secondary glioblastomas (Ohgaki and Kleihues 2013) and can be detected in approximately $10 \%$ of GBMs (Ohgaki and Kleihues 2012, Parsons et al. 2008). Patients of this tumor subgroup are usually younger at the time of diagnosis and have a longer overall survival. In the patient collective investigated in this study 5\% (6 of 120 cases) revealed an IDH-1 mutation. On average those patients were almost 16 years younger at the time of diagnosis (47.68 years compared to 63.03 years). Although slightly missing statistical significance, the IDH-1 mutated tumors showed a trend towards younger age $(\mathrm{p}=0.05063)$. There was no significant difference in longer overall survival (17.95 compared to 14.36 months, $\mathrm{p}=0.8276)$ and higher KPS at the time of diagnosis $(76.67 \%$ compared to $71.84 \%, \mathrm{p}=0.637)$. Multivariate analysis showed IDH-1 not to be an independent prognostic marker $(\mathrm{p}=0.9746)$. Even though the number of 6 cases seems to be too low to generate significant results, a non-significant difference in longer overall survival could be delineated while the role of age at the time of diagnosis corresponded with the findings in the literature.

It is the most prominent weakness of the tissue microarray method that only a small representative area of the tumor tissue can be assessed. Thus, the immunopositive area of a tumor tissue may have been missed and possibly led to a lower IDH-1 mutation rate in this population compared to the literature. However, in the case of IDH-1 this is unlikely, since the detection of mutations in immunohistochemistry is known to show a homogenous distribution among glial tumor cells. Furthermore, each tumor tissue sample was represented by two $2 \mathrm{~mm}$ samples, which is regarded as a large amount of tumor tissue compared to other studies using TMAs. A more likely cause of the lower rate of IDH-1 mutations could be that two secondary GBMs were excluded from the study due to differing adjuvant treatment regimens (no chemotherapy in one case and different radiotherapy in the other). One included case that was described as secondary GBM did not show an IDH-1 mutation. In all 6 cases that were IDH-1 mutated no precursor lesion in form of a lower graded glioma had been detected. It should be mentioned that the applied immunohistochemical staining method only detects the R132H mutation of IDH-1 which is by far the most common (approximately 90\%, see section 1.2.5). IDH-2 mutations can not be detected immunohistochemically and were not assessed in this study. Therefore the true number of IDH-mutated tumor samples may not have been reached. However, Ohgaki and Kleihues proclaim that IDH-1 mutation detection alone serves as a definite marker for 
Identification of Prognostically Relevant Cellular Markers of Differentiation in Glioblastoma

secondary GBM. This statement can not be supported or challenged with the data obtained (Ohgaki and Kleihues 2013). The inclusion criteria chosen for this patient collective do not allow a clear statement on this specific matter. The prognostic impact and the correlation with age and clinical status do partially correspond with the literature and are similar to the findings in secondary GBM. 


\subsubsection{NogoA}

NogoA (neurite outgrowth inhibitor A) is a member of the reticulon gene family, a group of proteins that are associated with the endoplasmic reticulum (GrandPre et al. 2000). It can be found in oligodendrogliomas (Marucci et al. 2012, Kuhlmann et al. 2008) and is believed to be a prognostic marker that correlates with lower grading (Xiong et al. 2007). Two studies showed that approximately $20 \%$ of GBMs hold high expression levels of NogoA (Marucci et al. 2012, Kuhlmann et al. 2008). Further investigations regarding the prognostic impact of NogoA in GBM have not yet been undertaken. In glioblastomas with oligodendroglial components (GBMOs) IDH1 mutation and MGMT promoter methylation are more frequently found. However, in previous studies GBMOs failed to show a better clinical course compared to GBMs in general (Ha et al. 2013, Wang et al. 2012, Vordermark 2006).

Nonetheless, the expression of NogoA in GBM and its correlation with clinical factors needed further detailed assessment. Marucci et al. and Kuhlmann et al., who assessed 30 and 29 GBMs, respectively, found approximately $20 \%$ to express NogoA. More specifically Kuhlmann et al. stated that $20 \%$ showed immunopositivity of more than $50 \%$ of the tumor cells (Marucci et al. 2012, Kuhlmann et al. 2008). The patient collective of 120 patients subject to this study showed high expression levels of NogoA (more than $50 \%$ of the tumor cells) in only $4.17 \%$.

Of the 3 cutoffs, that divided the patient collective in two groups with higher and lower NogoA expression levels, none showed a considerable difference in age at the time of diagnosis. A slight difference in clinical status in favor of higher NogoA expression rates was observed, although failing to reach statistical significance. Mean overall survival showed a notable, albeit non-significant difference for the first two cutoffs. Samples with none or very few scattered immunopositive cells had a survival advantage of two and a half months in comparison to higher expression rates (16.41 compared to 13.94 years, $\mathrm{p}=0.5420$ ). At the next cutoff the difference in overall survival was two months but also not statistically significant (15.75 compared to 13.73 years, for lower and higher expression rates, respectively, $\mathrm{p}=0.2708)$. Overall in our study NogoA neither shows a prognostic value in glioblastoma nor does it correlate with differences in clinical status or age. 


\subsubsection{OLIG2}

The oligodendrocyte transcription factor 2 (OLIG2) plays a major role in the structural development of the spinal cord. It promotes the differentiation of motor neurons and oligodendrocytes (Zhou and Anderson 2002) and is a member of the basic helixloop-helix protein (bHLH) family (Takebayashi et al. 2000, Zhou et al. 2000). OLIG2 is also linked to brain tumor development. It has been shown that OLIG2 expression is not restricted to tumors of oligodendroglial origin (Marie et al. 2001, Ligon et al. 2004). Some studies revealed a possible impact of OLIG2 in gliomagenesis through inhibition of tumor suppressor factors such as p21 and p53 (Mehta et al. 2011, Ligon et al. 2007, Ligon et al. 2004, Marie et al. 2001). No data has yet been published regarding the prognostic role of OLIG2 in patients suffering from glioblastoma and its possible correlations with other clinical findings.

The patient collective of this study was divided into two groups with differing OLIG2 expression levels at 5, 10, 20,30, 40 and 50\% of the tumor cells. Interestingly, at some cutoffs the subgroup with the lower expression rate had a lower clinical status at the time of diagnosis but without statistical significance. This was most pronounced at the $5 \%$ cutoff $(66.43 \%$ compared to $72.83 \%, \mathrm{p}=0.2048)$. This cutoff also showed a non-significant age difference (58.25 compared to 62.79 years, $\mathrm{p}=0.3217$ ), while all other cutoffs were approximately the same age. At all cutoffs the group with the lower expression level showed a tendency towards longer overall survival but never reached statistical significance. The cutoff at $30 \%$ barely missed the p-value of $5 \%(\mathrm{p}=0.0587)$. However, Cox regression analysis showed a statistically significant difference in overall survival for the OLIG2 cutoff at $30 \%(\mathrm{p}=0.0168$, see section 3.4$)$. Therefore, a prognostic impact of OLIG2 is likely and an expression rate below $30 \%$ of the tumor cells is a statistically significant independent positive prognostic factor according to the Cox regression analysis.

As delineated in section 4.3.2 it has been previously shown that glioblastoma multiforme with olidendroglioma component (GBMO) does not have a survival advantage besides MGMT promoter methylation and IDH-1 mutations being more frequent (Ha et al. 2013, Wang et al. 2012, Vordermark 2006). This may suggest that these two factors that usually correspond with longer overall survival in GBM have a decreased or no effect in GBMOs. As already mentioned in chapter 1.2.6, OLIG2 may even play a role in driving gliomagenesis through inhibition of tumor suppressor factors (Mehta et al. 2011, Ligon et al. 2007, Ligon et al. 2004, Marie et al. 2001). There is no data on the correlation of OLIG2 and GBMOs.

The data obtained in this study showed a significantly longer overall survival for tumor samples with expression levels of OLIG2 below 30\% in the multivariate analysis. Thus, glioblastomas with higher OLIG2 expression have a worse prognosis. This 
Identification of Prognostically Relevant Cellular Markers of Differentiation in Glioblastoma

indicates that the role of OLIG2 in gliomagenesis and its prognostic role in malignant gliomas needs further assessment. Considering the current efforts in molecular biological characterization of tumor subtypes, the method of immunohistochemistry applied in this study seems rather basic. However, the study revealed a prognostic subgroup of glioblastoma. A more detailed characterization of OLIG2-low GBMs is necessary, for example applying novel methylation arrays of GBMs. 


\subsubsection{Ki67}

As delineated in chapter 1.2.7, the prognostic role of Ki67 in astrocytoma has been subject to several studies. Some authors suggest the expression rate to be correlated with worse clinical outcome (Jin et al. 2011, Neder et al. 2004, Di et al. 1998, Sallinen et al. 1994) while other studies showed no impact of Ki67 as a prognostic marker (Vaquero et al. 2000, Litofsky et al. 1998, Chiba et al.2010, Berghoff et al. 2013). More specifically, the prognostic impact on glioblastoma also produced contradictory results. Two series of 38 and 37 GBMs investigated by Vaquero and Chiba, respectively, did not show Ki67 to have any prognostic impact (Chiba et al. 2010, Vaquero et al. 2000). In 2011 a larger study conducted by Jin et al. involved 156 glioblastomas. The cutoff was set at an expression rate of $25 \%$ of tumor cells. Individuals with increased expression rates (62.8\% of the samples) showed significantly shorter progression free and overall survival, while there was no difference in gender, age, extent of resection and KPS. Thus Jin et al. proclaimed Ki67 to be an independent prognostic marker (Jin et al. 2011).

In this study the expression rates of Ki67 showed a tendency towards Gaussian distribution. Approximately two thirds of the samples were assessed with 5-20\% immunopositive tumor cells (68.33\%), while $13.33 \%$ were above $20 \%$ and $17.50 \%$ below $5 \%$. This underlines that the cutoffs were well chosen. However, in this patient collective expression rates were not as high as described by Jin et al. in 2011. All three investigated cutoffs, chosen at 5\%,10\% and 20\%, did not reveal any considerable differences in age and KPS at the time of diagnosis or overall survival.

Due to the TMA method only small parts of the paraffin-embedded tumor tissue underwent immunohistochemical staining. Ki67 is known to show uneven expression levels throughout the glioblastoma tissue. It is possible that some samples did not represent an area of the tumor with sufficient Ki67 expression for immunohistochemical detection. This may explain the fact that expression levels of Ki67 were not as high as described in the literature (Jin et al. 2011). Additionally, most patients that were merely biopsied to confirm the diagnosis of GBM could not be included due to the small tumor sample size. This may have caused a selection bias. It is possible that these excluded cases were mainly patients with unfavorable clinical status that received a tumor biopsy since a subtotal or extensive resection was not feasible. In conclusion, the role of Ki67 as an independent prognostic marker as stated by Jin et al. can not be confirmed in the present study. However, the TMA procedure may not have been suitable to assess this immunohistochemical marker properly. 


\subsubsection{P53}

According to The Cancer Genome Atlas Research Network 87\% of primary GBMs have a mutation in the P53 pathway while $35 \%$ show a mutation of the actual gene TP53 (TCGA 2008). In the proneural subtype of glioblastoma, as proclaimed by Verhaak et al., 54\% show TP53 mutations. It is associated with younger age and shows a non-significant trend towards longer overall survival (Verhaak et al. 2010). The prognostic role of TP53 mutations in glioblastoma has been subject to several studies (Smith et al. 2001, Ohgaki et al. 2004, Batchelor et al. 2004, Felsberg et al. 2009). A significant prognostic impact could not be shown yet. It is believed that TP53 mutations are associated with younger age and therefore may lead to longer overall survival (Ohgaki et al. 2004, Batchelor et al. 2004).

As described in chapter 2, the immunohistochemical staining of p53 serves as an indirect detection of mutations of TP53. This patient collective showed p53 immunopositivity in $65.83 \%$ of the cases. Patients with p53 positive tumors held a significantly longer overall survival (15.88 compared to 11.95 months, $\mathrm{p}=0.0480$ ). However, as described by Ohgaki and Batchelor, in this patient collective the subgroup was also significantly younger $(\mathrm{p}=0.04054)$ and held a significantly better clinical status $(\mathrm{p}=0.01165)$ at the time of diagnosis (60.66 years and $74.68 \%$ compared to 65.16 years and $67.75 \%$ ). Multivariate analysis revealed that TP53 immunopositivity in general was not an independent prognostic marker. But a statistical trend was observed ( $\mathrm{p}=0.0689$ ). However, it was confirmed that the presence of TP53 positive tumor cells is associated with younger age and increased KPS. Both factors are known to correlate with longer overall survival, thus explaining the significant increase in overall survival in the Kaplan-Meier curve analysis.

As shown in chapter 3.3.5 none of the other p53 cutoffs $(5 \%, 10 \%, 20 \%, 30 \%)$ showed significant differences in mean overall survival in the Kaplan-Meier curve analyses or in age and clinical status in the independent tow-sample t-test. All cutoffs showed longer overall survival for the group with the higher rate of p53 positive cells. This difference increased with higher immunopositivity rates but never reached statistical significance in the Kaplan-Meier analysis. 


\subsubsection{GFAP}

GFAP plays a crucial role in the immunohistochemical diagnosis of brain tumors. Its expression rate in astrocytomas has been subject to several studies. It is believed that dedifferentiation of astrocytes is correlated with lower expression levels of GFAP (Wilhelmsson et al. 2003, Chen and Liem 1994, Rutka et al. 1994, Weinstein et al. 1991). In contrast other studies suggested a correlation of GFAP expression and WHO grading in astrocytomas (Reyaz et al. 2005, Heo et al. 2012). However, several studies showed no prognostic impact of GFAP expression in glioblastoma (Schmidt et al. 2002).

The cutoffs chosen in the present study did not show any statistically significant difference regarding overall survival as well as clinical status and age at diagnosis. A notable result was, however, shown at the first cutoff. A group of 14 samples showed a low expression rate of GFAP (majority of tumor cells remained unstained). These individuals showed a trend towards younger age at the time of diagnosis (54.99 compared to 63.39 years, $\mathrm{p}=0.07887)$, while $\operatorname{KPS}(\mathrm{p}=0.9028)$ and overall survival $(\mathrm{p}=0.3894)$ did not differ.

A possible correlation of younger age with low expression levels of GFAP, as shown with the first cutoff, has not yet been described in the literature. Even though the difference did not reach statistical significance, lower expression rates of GFAP showed a trend towards younger age. Due to the small number of patients (14) these findings need verification through further studies. It is possible that due to the TMA procedure the "true" GFAP expression of the tumor tissue was not assessed properly. However, this is unlikely since GFAP immunohistochemistry is known to show a highly homogeneous distribution. 


\subsubsection{Multivariate Analysis}

In order to reveal independent prognostic factors, multivariate analysis in form of the Cox regression model was applied. In contrast, the Kaplan-Meier survival analysis only focuses on one factor at a time. Possible confounding factors such as e.g. age differences of two observed groups were not taken into account. Thus the results of the multivariate analysis have a higher level of validity when it comes to assessing several variables.

Overall it is quite surprising that the well established independent prognostic factor age at the time of diagnosis failed statistical significance in the multivariate analysis of this study, although this factor showed significant differences in the Kaplan-Meier survival analysis. On the other hand, the variables that did reach statistical significance as independent prognostic factors (KPS and OLIG2 cutoff at 30\%) were in line with the obtained results from the Kaplan-Meier analysis. The clinical status is a well established prognostic factor that is routinely used to make treatment decisions for patients suffering from GBM. The impact of the extent of tumor resection on the overall survival is also well established (see chapter 1.2.1). It should be mentioned that multivariate analysis showed a strong trend towards EOR being an independent prognostic factor in this patient cohort.

The mutation of p53 has been shown to have an impact on overall survival in several studies. However, the survival advantage of p53 mutated GBMs is believed to be due to confounding factors such as younger age and better clinical status and therefore is not regarded as an independent prognostic factor (see section 1.2.9). In this patient cohort the expression of p53 was assessed immunohistochemically which serves as an indirect indicator for p53 mutations. However, the findings described in the literature were confirmed with the Kaplan-Meier analysis and the independent two sample t-test of this study. Interestingly, tumor samples with p53 immunopositivity showed statistical significance in the Kaplan-Meier survival analysis and merely a statistical trend in the multivariate analysis. This confirms the view expressed by other research groups (Smith et al. 2001, Ohgaki et al. 2004, Batchelor et al. in 2004).

A clear trend of OLIG2 expression below 30\% towards longer overall survival in the Kaplan-Meier survival analysis was observed. Multivariate analysis revealed this cutoff to be a significant independent prognostic factor, with lower expression levels having a better prognosis. At the time these findings can not be fully explained. However, reports about the role of OLIG2 as a glioma stem cell marker underline the correlation with worse prognosis of OLIG2 rich GBMs as delineated in this study. It also stresses the importance of this protein in future research endeavours of gliomagenesis (Trepant et al. 2014). 
Identification of Prognostically Relevant Cellular Markers of Differentiation in Glioblastoma 


\section{Chapter 5}

\section{Summary}

\section{Introduction:}

Glioblastoma multiforme is the most common primary brain tumor. Despite intense research efforts worldwide, the prognosis of patients diagnosed with this tumor remains poor. Several clinical and molecular parameters influence overall survival. However, apart from IDH-1, a prognostic marker that can be assessed immunohistochemically is still lacking. In this study the prognostic impact of several immunohistochemical markers was assessed in a cohort of 120 GBMs.

\section{Materials and Methods:}

In this study 120 tumor samples of patients diagnosed with glioblastoma multiforme underwent tissue microarray processing and subsequent immunohistochemical staining. All patients received adjuvant radiation and chemotherapy with an alkylating agent. The cellular expression of NogoA, OLIG2, GFAP, Ki67 and p53 and mutations of IDH-1 were assessed regarding their impact on overall survival, age and clinical status (KPS). Kaplan-Meier curve analysis (Gehan's Wilcoxon test), the independent two-sample t-test and multivariate analysis (Cox regression) were applied.

\section{Results:}

A small group of patients with low GFAP expression levels showed a tendency towards younger age at the time of diagnosis, a finding yet not described in the literature. The detection of IDH-1 mutations did not show a significant prognostic impact, but a clear trend towards younger age $(\mathrm{p}=0.05063)$. Immunopositivity of $\mathrm{p} 53$ was significantly associated with longer overall survival in the Kaplan-Meier analysis $(p=0.0480)$ and with younger age $(p=0.04054)$ and better clinical status $(p=0.01165)$. There was a strong trend towards longer overall survival of patients with lower expression levels of OLIG2, most pronounced at the cutoff at 30\%, $(\mathrm{p}=0.0587)$. 
Identification of Prognostically Relevant Cellular Markers of Differentiation in Glioblastoma

Interestingly, Cox regression analysis showed that expression rates of OLIG2 below $30 \%$ were a significant independent prognostic marker in glioblastoma multiforme $(\mathrm{p}=0.0168)$. The presence of $\mathrm{p} 53$ immunopositivity did not reach statistical significance in the multivariate analysis.

\section{Conclusion:}

In conclusion, the percentage of OLIG2 expressing tumor cells emerged as a prognostic factor for overall survival. Further studies are necessary to reveal its exact role in gliomagenesis. 


\section{Chapter 6}

\section{Bibliography}

Abaza MS, Shaban F, Narayan RK, Atassi MZ (1998): Human glioma associated intermediate filament proteins: over-expression, co-localization and cross-reactivity. Anticancer Res 18 (2B), 1333-1340

Ali Kahn A, O’Brien DF, Kelly P, Phillips JP, Rawluk D, Bolger C, Pidgeon CN (2007): The anatomical distribution of cerebral gliomas in mobile phone users. Ir Med J $\underline{96}$ (8), 240-242

Archavlis E, Tselis N, Birn G, Ulrich P, Baltas D, Zamboglou N (2013): Survival analysis of HDR brachytherapy versus reoperation versus temozolomide alone: a retrospective cohort analysis of recurrent glioblastoma multiforme. BMJ Open $\underline{3}$ (3), 1-9

Bailey P, Cushing H: A Classification of the Tumors of the Glioma Group on a Histogenetic Basis With a Correlated Study of Prognosis. JB Lippincott, Philadelphia 1926

Barnard RO, Geddes JF (1987): The incidence of multifocal cerebral gliomas. A histologic study of large hemisphere sections. Cancer 60 (7), 1519-1531

Batchelor TT, Betensky RA, Eposito JM, Pham LD, Dorfman MV, Piscatelli N, Jhung S, Rhee D, Louis DN (2004): Age-dependent prognostic effects of genetic alterations in glioblastoma. Clin. Cancer Res 10 (1), 228-233

Batchelor TT, Sorensen AG, Louis DN (2012): Case records of the Massachusetts General Hospital. Case 17-2012. A 54-year-old man with visual-field loss and a mass in the brain. N Engl J Med 366 (22), 2112-2120

Bauman GS, Gaspar LE, Fisher BJ, Halperin EC, Macdonald DR, Cairncross JG (1994): A prospective study of short-course radiotherapy in poor prognosis glioblastoma multiforme. Int J Radiat Oncol Biol Phys 29 (4), 835-839 
Berghoff AS, Stefanits H, Woehrer A, Heinzl. H, Preusser M, Hainfellner JA (2013): Clinical Neuropathology Practice Guide 3-2013: levels of evidence and clinical utility of prognostic and predictive candidate brain tumor biomarkers. Clin Neuropathol 32 (3), 148-158

Bock HC, Puchner MJ, Lohmann F, Schütze M, Koll S, Ketter R, Buchalla R, Rainaov N, Kantelhardt SR, Rohde V (2011): First-line treatment of malignant glioma with carmustine implants followed by concomitant radiochemotherapy: a multicenter experience. Neurosurg Rev 33 (4), 441-449

Bögler O, Huang HJ, Kleihues P, Cavenee WK (1995): The p53 gene and its role in human brain tumors. Glia 15 (3), 308-327

Boots-Sprenger SH, Sijben A, Rijntjes J, Tops BB, Idema AJ, Rivera AL, Bleeker FE, Gijtenbeek AN, Diefes K, Heathcock L (2013): Significance of complete 1p/19q co-deletion, IDH1 mutation and MGMT promoter methylation in gliomas: use with caution. Mod Pathol 26, 922-926

Brandes AA, Franceschi E, Tosoni A, Bartolini S, Bacci A, Agati R, Ghimenton C, Turazzi S, Talacchi A, Skrap M (2010): O(6)-methylguanine DNA-methyltransferase methylation status can change between first surgery for newly diagnosed glioblastoma and second surgery for recurrence: clinical implications.

Neuro-oncology $\underline{12}$ (3), 283-288

Brat DJ, Van Meir EG (2004): Vaso-occlusive and prothrombotic mechanisms associated with tumor hypoxia, necrosis, and accelerated growth in glioblastoma. Laboratory Investigation 84 (4), 397-405

Brat DJ, Seiferheld WF, Perry A, Hammond EH, Murray KJ, Schulsinger AR, Mehta MP, Curran WJ (2004): Analysis of 1p, 19q, 9p, and 10q as prognostic markers for high-grade astrocytomas using fluorescence in situ hybridization on tissue microarrays from Radiation Therapy Oncology Group trials. Neuro-oncology $\underline{6}(2), 96-103$

Braun N, Papadopoulos T, Müller-Hermelink HK (1988): Cell cycle dependent distribution of the proliferation-associated Ki-67 antigen in human embryonic lung cells. Virchows Arch., B, Cell Pathol 56 (1), 25-33 
Brem H, Piantadosi S, Burger PC, Walker M, Selker R, Vick NA, Black K, Sisti M, Brem S, Mohr G (1995): Placebo-controlled trial of safety and efficacy of intraoperative controlled delivery by biodegradable polymers of chemotherapy for recurrent gliomas. The Polymer-brain Tumor Treatment Group. Lancet 345 (8956), 1008-1012

Brommeland T, Rosengren L, Fridlund S, Henning R, Isaksen V (2007): Serum levels of glial fibrillary acidic protein correlate to tumour volume of high-grade gliomas. Acta Neurol Scand 116 (6), 380-384

Bullwinkel J, Baron-Lühr B, Lüdemann A, Wohlenberg C, Gerdes J, Scholzen T (2006): Ki-67 protein is associated with ribosomal RNA transcription in quiescent and proliferating cells. J Cell Physiol 206 (3), 624-635

Burger PC, Dubois PJ, Schold SC, Smith KR, Odom GL, Crafts DC, Giangaspero F (1983): Computerized tomographic and pathologic studies of the untreated, quiescent, and recurrent glioblastoma multiforme. J Neurosurg 58 (2), 159-169

Busch EA (1963): Indications for surgery in glioblastomas. Clin Neurosurg 9, 1-17

Cahill DP, Levine KK, Betensky RA, Codd PJ, Romany CA, Reavie LB, Batchelor TT, Futreal PA, Stratton MR, Curry WT (2007): Loss of the mismatch repair protein MSH6 in human glioblastomas is associated with tumor progression during temozolomide treatment. Clin Cancer Res 13 (7), 2038-2045

Cairncross G, Berkey B, Shaw E, Jenkins R, Scheithauer B, Brachman D, Buckner J, Fink K, Souhami L, Laperierre N (2006): Phase III trial of chemotherapy plus radiotherapy compared with radiotherapy alone for pure and mixed anaplastic oligodendroglioma: Intergroup Radiation Therapy Oncology Group Trial 9402. J Clin Oncol 24 (18), 2707-2714

Caloglu M, Yurut-Caloglu V, Karagol H, Bayir-Angin G, Turan FN, Uzal C (2009): Prognostic factors other than the performance status and age for glioblastoma multiforme: a single-institution experience. J BUON 14 (2), 211-218

Carlson MR, Pope WB, Horvath S, Braunstein JG, Nghiemphu P, Tso CL, Mellinghoff I, Lai A, Liau LM, Mischel PS (2007): Relationship between survival and edema in malignant gliomas: role of vascular endothelial growth factor and neuronal pentraxin 2. Clin Cancer Res 13 (9), 2592-2598 
Catalan-Uribarrena G, Bilbao-Barandica G, Pomposo-Gaztelu I, Undabeitia-Huertas J, Ruiz de Gopegui-Ruiz E, Galbarriatu-Gutirrez L, Canales-Llantada M, Aurrecoechea-Obieta J, Igartua-Azkune A,Carbayo-Lozano G (2012): Prognostic factors and survival in a prospective cohort of patients with high-grade glioma treated with carmustine wafers or temozolomide on an intention-to-treat basis. Acta Neurochir $\underline{154}$ (2), 211-222

Chandana SR, Movva S, Arora M, Singh T (2008): Primary brain tumors in adults. Am Fam Physician 77 (10), 1423-1430

Chang SM, Parney IF (2005): Patterns of care for adults with newly diagnosed malignant glioma. JAMA 293 (5), 557-564

Chen SM, Huber AB, van der Haar ME, Frank M, Schnell L, Spillmann AA, Christ F, Schwab ME (2000): Nogo-A is a myelin-associated neurite outgrowth inhibitor and an antigen for monoclonal antibody IN-1. Nature $\underline{403}$ (6768), 434-439

Chen WJ, Liem RK (1994): Reexpression of glial fibrillary acidic protein rescues the ability of astrocytoma cells to form processes in response to neurons. J Cell Biol $\underline{127}$ (3), 813-823

Chiba Y, Hashimoto N, Tsuboi A, Rabo C, Oka Y, Kinoshita M, Kagawa N, Oji Y, Sugiyama H, Yoshimine T (2010): Prognostic value of WT1 protein expression level and MIB-1 staining index as predictor of response to WT1 immunotherapy in glioblastoma patients. Brain Tumor Pathol 27 (1), 29-34

Chill DP, Levine KK, Betensky RA, Codd PJ, Romany CA, Reavie LB, Batchelor TT, Futreal PA, Stratton MR (2007): Loss of the mismatch repair protein MSH6 in human glioblastomas is associated with tumor progression during temozolomide treatment. Clin Cancer Res 13 (7), 2038-2045

Chin HW, Lefkowitz DM, Eisenberg RL (1992): Treatment options in high-grade brain tumors: brain brachytherapy. Radiographics 12 (4), 721-729

Chinot OL, Barrie M, Frauger E, Dufour H, Figarella-Branger D, Palmari J, Braguer D, Hoang-Xuan K, Moktari K, Peragut JC (2004): Phase II study of temozolomide without radiotherapy in newly diagnosed glioblastoma multiforme in an elderly populations. Cancer 100 (10), 2208-2214

Choucair AK, Levin VA, Gutin PH, Davis RL, Silver P, Edwards MS, Wilson CB (1986): Development of multiple lesions during radiation therapy and chemotherapy in patients with gliomas. J Neurosurg $\underline{65}$ (5), 654-658 
Cohen MH, Shen YL, Keegan P, Pazdur R (2009): FDA drug approval summary: bevacizumab (Avastin) as treatment of recurrent glioblastoma multiforme.

Oncologist 14 (11), 1131-1138

Combs SE, Rieken S, Wick W, Abdollahi A, von Deimling A, Debus J, Hartmann C (2011): Prognostic significance of IDH-1 and MGMT in patients with glioblastoma: one step forward, and one step back? Radiat Oncol 6, 115

Conte V, Baratta P, Tomaselli P, Songa V, Magni L, Stocchetti N (2008): Awake neurosurgery: an update. Minerva Anestesiol 74 (6), 289-292

Crowley RW, Pouratian M, Sheehan JP (2006): Gamma knife surgery for glioblastoma multiforme. Neurosurg. Focus 20 (4), E17

Di X, Nishizaki K, Harada K, Kajiwara K, Nakayama H, Ito H (1997): Proliferative potentials of glioma cells and vascular components determined with monoclonal antibody MIB-1. J Exp Clin Cancer Res 16 (4), 389-394

Dolecek TA, Propp JM, Stroup NE, Kruchko C (2012): CBTRUS Statistical Report: Primary Brain and Central Nervous System Tumors Diagnosed in the United States in 2005-2009. Neuro Oncol 14 (Suppl 5), v1-v49

Ekstrand AJ, Sugawa N, James CD, Collins VP (1992): Amplified and rearranged epidermal growth factor receptor genes in human glioblastomas reveal deletions of sequences encoding portions of the N- and/or C-terminal tails. Proc Natl Acad Sci USA 89 (10), 4309-4313

Eng LF, Vanderhaeghen JJ, Bignami A, Gerstl B (1971): An acidic protein isolated from fibrous astrocytes. Brain Res 28 (2), 351-354

Eng LF, Ghirnikar RS, Lee YL (2000): Glial fibrillary acidic protein: GFAP-thirty-one years (1969-2000). Neurochem Res 25 (9-10), 1439-1451

Erickson KM, Cole DJ (2012): Anesthetic considerations for awake craniotomy for epilepsy and functional neurosurgery. Anesthesiol Clin 30 (2), 241-268

Erlich SS, Davis RL (1978): Spinal subarachnoid metastasis from primary intracranial glioblastoma multiforme. Cancer 42 (6), 2854-2864

Esteller M, Garcia-Foncillas J, Andion E, Goodman SN, Hidalgo OF, Vanaclocha V, Baylin SB, Herman JG (2000): Inactivation of the DNA-repair gene MGMT and the clinical response of gliomas to alkylating agents. N Engl J Med 343 (19), 1350-1354 
Fan X, Salford LG, Widegren B (2007): Glioma stem cells: evidence and limitation. Semin Cancer Biol 17 (3), 214-218

Felsberg J, Erkwoh A, Sabel MC, Kirsch L, Fimmrs R, Blaschke B, Schlegel U, Schramm J, Wiestler OD, Reifenberger G (2004): Oligodendroglial tumors: refinement of candidate regions on chromosome arm $1 \mathrm{p}$ and correlation of $1 \mathrm{p} / 19 \mathrm{q}$ status with survival. Brain Pathol 14 (2), 121-130

Felsberg J, Rapp M, Loeser S, Fimmers R, Stummer W, Goeppert M, Steiger HJ, Friedensdorf B, Reifenberger G, Sabel MC (2009): Prognostic significance of molecular markers and extent of resection in primary glioblastoma patients. Clin Cancer Re 15 (21), 6683-6693

Fisher JL, Schwarzbaum JA, Wrensch M, Berger MS (2006): Evaluation of epidemiologic evidence for primary adult brain tumor risk factors using evidence-based medicine. Prog Neuro Surg 19, 54-79

Fodstad H, Kelly PJ, Buchfelder M (2006): History of the cushing reflex. Neurosurgery 59 (5), 1132-1137

Forsyth PA, Posner JB (1993): Heachaches in patients with brain tumors: a study of 111 patients. Neurology $\underline{43}$ (9), 1678-1683

Fournier AE, GrandPre T, Strittmatter SM (2001): Identification of a receptor mediating Nogo-66 inhibition of axonal regeneration. Nature 409 (6818), 341-346

Frankel SA, German WJ (1953): Glioblastoma multiforme; review of 219 cases with regard to natural history, pathology, diagnostic methods, and treatment. J Neurosurg 15 (5), 489-503

Fu J, Koul D, Yao J, Wang S, Yuan Y, Colman H, Sulman EP, Lang FF, Yung WA (2013): Novel HSP90 inhibitor NVP-HSP990 targets cell-cycle regulators to ablate Olig2-positive glioma tumor-initiating cells. Cancer Res 73 (10), 3062-3074

Galacich JH, French LA, Melby JC (1961): Use of dexamethasone in treatment of cerebral edema associated with brain tumors. J Lancet $81,46-53$

Garside R, Pitt M, Anderson R, Roger G, Dyer M, Mealing S, Somerville M, Price A, Stein K (2007): The effectiveness and cost-effectiveness of carmustine implants and temozolomide for the treatment of newly diagnosed high-grade glioma: a systematic review and economic evaluation. Health Technol Assess 11 (45), iii-iv, ix-221 
Gaspar LE, Fisher BJ, Macdonald DR, LeBer DV, Halperin EC, Schold SC, Cairncross JD (1992): Supratentorial malignant glioma: patterns of recurrence and implications for external beam local treatment. Int. J Radiat Oncol Biol Phys 24 (1), 55-57

Geer CP, Grossman SA (1997): Interstitial fluid flow along white matter tracts: a potentially important mechanism for the dissemination of primary brain tumors. J Neurooncol 32 (3), 193-201

Gerdes J, Schwab U, Lemke H, Stein H (1983): Production of a mouse monoclonal antibody reactive with a human nuclear antigen associated with cell proliferation. Int J Cancer 31 (1), 13-20

Giese A, Bjerkvig R, Berens ME, Westphal M (2003): Cost of Migration: Invasion of Malignant Gliomas and Implications for Treatment. JCO 21 (8), 1624-1636

Glasgow SM, Zhu W, Stolt CC, Huang TW, Chen F, LoTurco JJ, Neul JL, Wegner M, Mohila C, Deneen B (2014): Mutual antagonism between Sox10 and NFIA regulates diversification of glial lineages and glioma subtypes. Nat Neurosci 17 (10), $1322-9$

Golgi C: Untersuchungen über den feineren Bau des zentralen und peripheren Nervensystems. Fischer, Jena 1894

Gomez-Manzano C, Fueyo J, Kyritsis AP, McDonnell TJ, Steck PA, Levin VA, Yung WK (1997): Characterization of p53 and p21 functional interactions in glioma cells en route to apoptosis. J Natl Cancer Inst 89 (14), 1036-1044

GrandPre T, Nakamura F, Vartanian T, Strittmatter SM (2000): Identification of the Nogo inhibitor of axon regeneration as a Reticulon protein. Nature 403 (6768), 439-444

Greenberg MS: Handbook of Neurosurgery. 7th edition; Thieme, Stuttgart 2012 Gulati S, Jakola AS, Nerland US, Weber C, Solheim O (2011): The risk of getting worse: surgically acquired deficits, perioperative complications, and functional outcomes after primary resection of glioblastoma. World Neurosurg $\underline{76}$ (6), 572-579

Ha SY, Kang SY, Do IG, Suh YL (2013): Glioblastoma with oligodendroglial component represents a subgroup of glioblastoma with high prevalence of IDH1 mutation and association with younger age. J Neurooncol 112 (3), 439-448 
Habberstad AH, Lind-Landstrm T, Sundstrm S, Torp SH (2012): Primary human glioblastomas - prognostic value of clinical and histopathological parameters. Clin Neuropathol 31 (5), 361-368

Hamaya K, Doi K, Tanaka T, Nishimoto A (1985): The determination of glial fibrillary acidic protein for the diagnosis and histogenetic study of central nervous system tumors: a study of 152 cases. Acta Med Okayama 39 (6), 453-462

Hamer PC, Robles SG, Zwinderman AH, Duffau H, Berger MS (2012): Impact of Intraoperative Stimulation Brain Mapping on Glioma Surgery Outcome: A Meta-Analysis. JCO 30 (20), 2559-2565

Hamilton MG, Hull RD, Pineo GF (1994): Prophylaxis of venous thromboembolism in brain tumor patients. J Neurooncol 22 (2), 111-126

Han SJ, Yang I, Tihan T, Prados MD, Parsa AT (2010): Primary gliosarcoma: key clinical and pathologic distinctions from glioblastoma with implications as a unique oncologic entity. J Neurooncol 96 (3), 313-320

Hart MG, Grant R, Garside R, Rogers G, Somerville M, Stein K (2011): Chemotherapy wafers for high grade glioma. Cochrane Database Syst Rev $\underline{3}$, CD007294

Hartmann C, Meyer J, Balss J, Capper D, Mueller W, Christians A, Felsberg J, Wolter M, Mawrin C, Wick W (2009): Type and frequency of IDH1 and IDH2 mutations are related to astrocytic and oligodendroglial differentiation and age: a study of 1,010 diffuse gliomas. Acta Neuropathol 118 (4), 469-474

Heesters M, Koudstaal J, Go KG, Molenaar WM (1999): Analysis of Proliferation and Apoptosis in Brain Gliomas: Prognostic and Clinical Value. J Neurooncol 44 (3), 255-266

Hegi ME, Diserens AC, Gorlia T, Hamou MF, de Tribolet N, Weller M, Kros JM, Hainfellner JA, Mason W, Maiana L (2005): MGMT gene silencing and benefit from temozolomide in glioblastoma. N Engl J Med $\underline{352}$ (10), 997-1003

Hegi ME, Liu L, Herman JG, Stupp R, Wick W, Weller M, Mehta MP, Gilbert MR (2008): Correlation of O6-Methylguanine Methyltransferase (MGMT) Promoter Methylation With Clinical Outcomes in Glioblastoma and Clinical Strategies to Modulate MGMT Activity. JCO 26 (25), 4189-4199

Heo DH, Kim SH, Yang KM, Cho YJ, Kim KN, Yoon DH, Kang TC (2012): A histopathological diagnostic marker for human spinal astrocytoma: expression of glial fibrillary acidic protein- $\delta$. J Neurooncol 108 (1), 45-52 
Hochberg FH, Pruitt A (1980): Assumptions in the radiotherapy of glioblastoma. Neurology 30 (9), 907-911

Holland EC (2000). Glioblastoma multiforme: The terminator. PNAS 97 (12), $6242-6244$

Hollstein M, Sidransky D, Vogelstein B, Harris CC (1991): p53 mutations in human cancers. Science 253 (5015), 49-53

Horsted F, West J, Grainge MJ (2012): Risk of venous thromboembolism in patients with cancer: a systematic review and meta-analysis. PloS Med 9 (7), e1001275

Hurwitz H, Fehrenbach L, Novotny W, Cartwright T, Hainsworth J, Heim W, Berlin J, Baron A, Griffing S, Holmgren E (2004): Bevacizumab plus irinotecan, fluorouracil, and leucovorin for metastatic colorectal cancer. N Engl J Med 350 (23), 2335-2342

Isoardo G, Morra I, Chiarle G, Audrito V, Deaglio S, Melcarne A, Junemann C, Naddeo M, Cogoni M, Valentini MC (2012): Different Aquaporin-4 Expression in Glioblastoma Multiforme Patients with and without Seizures. Mol Med 18 (1), $1147-1151$

Jacobs AH, Kracht LW, Gossmann A, Rger MA, Thomas AV, Thiel A, Herholz Karl (2005): Imaging in Neurooncology. NeuroRx 2 (2), 333-347

Jacque CM, Vinner C, Kujas M, Raoul M, Racadot J, Baumann NA (1978):

Determination of glial fibrillary acidic protein (GFAP) in human brain tumors. J Neurol Sci 35 (1), 147-155

Jenkins RB, Blair H, Ballman KV, Giannini C, Arusell RM, Law M, Flynn H, Passe S, Felten S, Brown PD (2006): A t $(1 ; 19)$ (q10;p10) mediates the combined deletions of $1 p$ and $19 q$ and predicts a better prognosis of patients with oligodendroglioma. Cancer Res 66 (20), 9852-9861

Jenkinson MD, Du Plessis DG, Walker T, Smith TS (2007): Advanced MRI in the management of adult gliomas. Br J Neurosurg 21 (6), 550-561

Jin Q, Zhang W, Qui XG, Yan W, You G, Liu YW, Jiang T, Wang L (2011): Gene expression profiling reveals Ki-67 associated proliferation signature in human glioblastoma. Chin Med J 124 (17), 2584-2588

Johannessen AL, Torp SH (2006): The clinical value of Ki-67/MIB-1 labeling index in human astrocytomas. Pathol Oncol Res 12 (3), 143-147 
Johnson DR, Ma DJ, Buckner JC, Hammack JE (2012): Conditional probability of long-term survival in glioblastoma. Cancer 118 (22), 5608-5613

Jones DT, Gronych J, Lichter P, Witt O, Pfister SM (2012): MAPK pathway activation in pilocytic astrocytoma. Cell Mol Life Sci 69 (11), 1799-1811

Jung CS, Foerch C, Schänzer A, Heck A, Plate KH, Seifert V, Steinmetz H, Raabe A, Sitzer M (2007): Serum GFAP is a diagnostic marker for glioblastoma multiforme. Brain 130 (Pt 12), 3336-3341

Kalkanis SN, Carroll RS, Zhang J, Zamani AA, Black PM (1996): Correlation of vascular endothelial growth factor messenger RNA expression with peritumoral vasogenic cerebral edema in meningiomas. J Neurosurg 85 (6), 1095-1101

Kaneshiro D, Kobayashi T, Chao ST, Suh J, Prayson RA (2009): Chromosome 1p and 19q deletions in glioblastoma multiforme. Appl. Immunohistochem. Mol Morphol 17 (6), 512-516

Karamitopoulou E, Perentes E, Diamantis I, Maraziotis T (1994): Ki-67 immunoreactivity in human central nervous system tumors: a study with MIB 1 monoclonal antibody on archival material. Acta Neuropathol 87 (1), 47-54

Kargiotis O, Markoula S, Kyritsis AP (2011): Epilepsy in the cancer patient. Cancer Chemother Pharmacol 67 (3), 489-501

Khalid H, Shibata S, Kishikawa W, Yasunaga A, Iseki M, Hiura T (1997): Immunohistochemical analysis of progesterone receptor and Ki-67 labeling index in astrocytic tumors. Cancer 80 (11), 2133-2140

Kirste S, Treier M, Wehrle SJ, Becker G, Abdel-Tawab M, Gerbeth K, Hug MJ, Lubrich B, Grosu AL, Momm F (2011): Cancer 117 (16), 3788-3795

Koch G: Bildmorphologische Parameter properativer MR-Tomogramme und berlebenszeit von Patienten mit malignen Gliomen. Med. Diss. Berlin 2007

Kozac KR, Moody JS (2009): Giant cell glioblastoma: A glioblastoma subtype with distinct epidemiology and superior prognosis. Neuro Oncol 11 (6), 833-841

Kreisl TN, Kim L, Moore K, Duic P, Royce C, Stroud I, Garren N, Mackey M, Butman JA, Camphausen K (2009): Phase II trial of single-agent bevacizumab followed by bevacizumab plus irinotecan at tumor progression in recurrent glioblastoma. J Clin Oncol 27 (5), 740-745 
Krex D, Klink B, Hartmann C, von Diemling A, Pietsch T, Simon M, Sabel M, Steinbach JT, Heese O, Reifenberger G (2007): Long-term survival with glioblastoma multiforme. Brain 130 (pt10), 2596-2606

Kuhlmann T, Gutenberg A, Schulten HJ, Paulus W, Rohde V, Bruck W (2008): Nogo-a expression in glial CNS tumors: a tool to differentiate between oligodendrogliomas and other gliomas? Am J Surg Pathol 23 (10), 1444-1453

Lacroix M, Abi-Said D, Fourney DA, Gokaslan ZR, Shi W, DeMonte F, Lang FF, McCutcheon IE, Hassenbusch SJ, Holland E (2001): A multivariate analysis of 416 patients with glioblastoma multiforme: prognosis, extent of resection, and survival. J Neurosurg $\underline{95}$ (2), 190-198

Lamszus K, Ulbricht U, Matschke J, Brockmann MA, Fillbrandt R, Westphal M (2003): Levels of soluble vascular endothelial growth factor (VEGF) receptor 1 in astrocytic tumors and its relation to malignancy, vascularity, and VEGF-A. Clin Cancer Res $\underline{9}$ (4), 1399-1405

Lane DP (1992): Cancer. p53, guardian of the genome. Nature 358 (6381), 15-16 Laperriere N, Zuraw L, Cairncross G (2002): Radiotherapy for newly diagnosed malignant glioma in adults: a systematic review. Radiother Oncol 64 (3), 259-273

Larjavaara S, Mantyla R, Salminen T, Haapasalo H, Raitanen J, Jaaskelainen J, Auvinen A (2007): Incidence of gliomas by anatomic location. Neuro Oncol 9 (3), 319-325

Laws ER, Parney IF, Huang W, Anderson F, Morris AM, Asher A, Lillehei KO, Bernstein M, Brem M, Sloan A (2003): Survival following surgery and prognostic factors for recently diagnosed malignant glioma: data from the Glioma Outcomes Project. J Neurosurg $\underline{99}$ (3), 467-473

Lee JW, Wen PY, Hurwitz S, Black P, Kesari S, Drappatz J, Golby AJ, Wells WM, Warfiled SK, Kikinis R, Bromfield EB (2012): Morphological Characteristics of Brain Tumors Causing Seizures. Arch Neurol 67 (3) 336-342

Ligon KL, Alberta JA, Kho AT, Weiss J, Kwaan MR, Nutt CL, Louis DN, Stiles CD, Rowitch DH (2004): J Neuropathol Exp Neurol 63 (5), 499-509

Ligon KL, Huillard E, Mehta S, Kesari S, Liu H, Alberta JA, Bachoo RM, Kane M, Louis DM, Depinho RA (2007): Olig2-regulated lineage-restricted pathway controls replication competence in neural stem cells and malignant gliomas. Neuron 53 (4), 503-517 
Litofsky NS, Mix TC, Baker SP, Recht LD, Smith TW (1998): Ki-67 (clone MIB-1) proliferation index in recurrent glial neoplasms: no prognostic significance. Surgical Neurology 50 (6), 579-585

Louis DN, Ohgaki H, Wiestler OD, Cavenee WK, Burger PC, Jouvet A, Scheihauer BW, Kleihues P (2007): The 2007 WHO Classification of Tumors of the Central Nervous System. Acta Neuopathol 114 (2), 97-109

Lutterbach J, Guttenberg R, Pagenstecher A (2001): Gliosarcoma: a clinical study. Radiother Oncol 61 (1), 57-64

Machein MR, Plate KH (2000): VEGF in brain tumors. J Neurooncol 50 (1-2), $109-120$

Maiuri F, Stella L, Benvenuti D, Giamundo A, Pettinato G (1990): Cerebral gliosarcomas: correlation of computed tomographic findings, surgical aspect, pathological features, and prognosis. Neurosurgery 26 (2), 261-267

Marie Y, Sanson M, Mokhtari K, Leuraud P, Kujas M, Delattre JY, Poirier J, Zalc B, Hoang-Xuang K (2001): OLIG2 as a specific marker of oligodendroglial tumour cells. Lancet 358 (9278), 298-300

Marina O, Suh JH, Reddy CA, Barnett GH, Vogelbaum MA, Peereboom DM, Stevens GH, Elinzano H, Chao ST (2011): Treatment outcomes for patients with glioblastoma multiforme and a low Karnofsky Performance Scale score on presentation to a tertiary care institution. J Neurosurg 115 (2), 220-229

Marucci G, Di Oto E, Farnedi A, Panzacchi R, Ligorio C, Foschini MP (2012): Nogo-A: a useful marker for the diagnosis of oligodendroglioma and for identifying 1p19q codeletion. Hum Pathol 43 (3), 374-380

Maschio M (2012): Brain Tumor-Related Epilepsy. Curr Neuopharmacol 10 (2), 124-133

Maslehaty H, Cordovi S, Hefti M (2011): Symptomatic spinal metastases of intracranial glioblastoma: clinical characteristics and pathomechanism relating to GFAP expression. J Neurooncol 101 (2), 329-333

McGirt MJ, Than KD, Weingart JD, Chaichana KL, Attenello FJ, Olivi A, Laterra J, Kleinberg LR, Grossman SA, Brem H (2009a): Gliadel (BCNU) wafer plus concomitant temozolomide therapy after primary resection of glioblastoma multiforme. J Neurosur 110 (3), 583-588 
McGirt MJ, Mukherjee D, Chaichana HL, Than KD, Weingart JD, Quinones-Hinojosa A (2009b): Association of surgically acquired motor and language deficits on overall survival after resection of glioblastoma multiforme. Neurosurgery 65 (3), 463-470

Mehta S, Huillard E, Kesari S, Maire CL, Golebiowski D, Harrington EP, Alberta JA, Kane MF, Theisen M, Ligon KL (2011): The central nervous system restricted transcription factor Olig2 opposes p53 responses to genotoxic damage in neural progenitors and malignant glioma. Cancer Cell 19 (3), 359-371

Meijer DH, Kane MF, Mehta F, Liu H, Harrington E, Taylor CM, Stiles CD, Rowitch DH (2012): Separated at birth? The functional and molecular divergence of OLIG1 and OLIG2. Nat Rev Neurosci 13 (12), 819-831

Menezes DL, Taverna P, Jensen MR, Abrams T, Stuart D, Yu GK, Duhl D, Machajewski T, Sellers WR, Pryrer NK (2012): The novel oral Hsp90 inhibitor NVP-HSP990 exhibits potent and broad-spectrum antitumor activities in vitro and in vivo. Mol Cancer Ther 11 (3), 730-739

Middeldorp J, Hol EM (2011): GFAP in health and disease. Prog Neurobiol 93 (3), 421-443

Miller CR, Perry A (2007): Glioblastoma. Arch Pathol Lab Med 131 (3), 397-406

Mor V, Laliberte L, Morris JN, Wiemann M (1984): The Karnofsky Performance Status Scale. An examination of its reliability and validity in a research setting. Cancer 53 (9), 2002-2007

Narayana A, Gruber D, Kunnakkat S, Golfinos JG, Parker E, Raza S, Zagzag D, Eagan P, Gruber ML (2012): A clinical trial of bevacizumab, temozolomide, and radiation for newly diagnosed glioblastoma. J Neurosurg 116 (2), 341-345

Neder L, Colli BO, Machado HR, Carlotti CG, Santos AC, Chimelli L (2004): MIB-1 labeling index in astrocytic tumors-a clinicopathologic study. Clin Neuropathol 23 (6), 262-270

Newton HB (1994): Primary brain tumors: review of etiology, diagnosis and treatment. Am Fam Physician 49 (4), 787-797

Ohgaki H, Kleihues P (2007): Genetic Pathways to Primary and Secondary Glioblastoma. Am J Pathol 170 (5), 1445-1453

Ohgaki H, Kleihues P (2009): Genetic alterations and signaling pathways in the evolution of gliomas. Cancer Sci 100 (12), 2235-2241 
Ohgaki H, Kleihues P (2012): The definition of primary and secondary glioblastoma. Clin Cancer Res 19 (4), 764-772

Ohgaki H, Dessen P, Jourde B, Horstmann S, Nishikawa T, Di Patre PL, Burkhard C, Schueler D, Probst-Hensch NM, Maiorka PC (2004): Genetic pathways to glioblastoma: a population-based study. Caner Res $\underline{64}$ (19), 6892-6899

Onishi A, Sawa H, Tsuda M, Sawamura Y, Itoh T, Iwasaki Y, Nagashima K (2003): Expression of the oligodendroglial lineage-associated markers Olig1 and Olig2 in different types of human gliomas. J Neuropathol Exp Neurol 62 (10), 1052-1059

Orzaiz GA, Solis ST, Valverde EP, Sanchez MM, Herruzo BB, Idoate Gastearena M, Valle RD (2013): Prognostic Value of Residual Fluorescent Tissue in Glioblastoma Patients After Gross Total Resection in 5-ALA Guided Surgery. Neurosurgery 154 (11), 1997-2002

Palma L, Celli P, Maleci A, Di Lorenzo N, Cantore G (1989): Malignant monstrocellular brain tumours. A study of 42 surgically treated cases. Acta Neurochir 97 (1-2), 17-25

Parsons DW, Jones S, Zhang X, Lin JC, Leary RJ, Angenendt P, Mankoo P, Carter H, Siu IM, Gallia GL (2008): An integrated genomic analysis of human glioblastoma multiforme. Science 321 (5897), 1807-1812

Peereboom DM, Shepard DR, Ahluwalia MS, Brewer CJ, Agarwal N, Stevens GH, Suh JH, Toms SA, Vogelbaum MA, Weil RJ (2010): Phase II trial of erlotinib with temozolomide and radiation in patients with newly diagnosed glioblastoma multiforme. J Neurooncol 98 (1), 93-99

Perry J, Chambers A, Spithoff K, Laperriere N (2007): Gliadel wafers in the treatment of malignant glioma: a systematic review. Curr Oncol 14 (5), 189-194

Perry JR (2012): Thromboembolic disease in patients with high-grade glioma.

Neuro-oncology 14 (4), iv73-80

Picard D (2002): Heat-shock protein 90, a chaperone for folding and regulation. Cell Mol Life Sci 59 (10), 1640-1648

Pinto LW, Araujo MB, Vettore AL, Wernersbach L, Leite AC, Chimelli LA, Soares FA (2008): Glioblastomas: correlation between oligodendroglial components, genetic abnormalities, and prognosis. Virchows Arch $\underline{452}$ (5), 481-490 
Reyaz N, Tayyab M, Khan SA, Siddique T (2005): Correlation of glial fibrillary acidic protein (GFAP) with grading of the neuroglial tumours. J Coll Physicians Surg Pak 15 (8), 472-475

Rhamanzadeh R, Hüttmann G, Gerdes J, Scholzen T (2007): Chromophore-assisted light inactivation of pKi-67 leads to inhibition of ribosomal RNA synthesis. Cell Prolif 40 (3), 422-430

Roa W, Brasher PM, Bauman G, Anthes M, Bruera E, Chan A, Fisher B, Fulton D, Gulavita S, Hao C (2004): Abbreviated course of radiation therapy in older patients with glioblastoma multiforme: a prospective randomized clinical trial. J Clin Oncol $\underline{22}$ (9), 1583-1588

Robert Koch Institute: Krebs in Deutschland 2007/2008. 8. Ausgabe. Robert Koch-Institut (Hrsg) und die Gesellschaft der epidemiologischen Krebsregister in Deutschland e.V. (Hrsg). Robert Koch Institut Berlin, 2012

Roessmann U, Velasco ME, Sindely SD, Gambetti P (1980): Glial fibrillary acidic protein (GFAP) in ependymal cells during development. An immunocytochemical study. Brain Res 200 (1), 13-21

Rogers G, Garside R, Mealing S, Pitt M, Anderson R, Dyer M, Stein K, Somerville M (2008): Carmustine implants for the treatment of newly diagnosed high-grade gliomas: a cost-utility analysis. Pharmacoeconomics 26 (1), 33-44

Romanelli P, Conti A, Pontoriero A, Ricciardi GK, Tomasello F, De Renzis C, Innocenzi G, Esposito V, Cantore G (2009): Role of stereotactic radiosurgery and fractionated stereotactic radiotherapy for the treatment of recurrent glioblastoma multiforme. Neurosurg Focus 27 (6), E8

Rong Y, Durden DL, Van Meir EG, Brat DJ (2006): 'Pseudopalisading' necrosis in glioblastoma: a familiar morphologic feature that links vascular pathology, hypoxia, and angiogenesis. J Neuropathol Exp Neurol 65 (6), 529-539

Roth JG, Elvidge AR (1960): Glioblastoma multiforme: a clinical survey. J Neurosurg 17, 736-750

Rutka JT, Hubbard SL, Fukuyama K, Matsuzawa K, Dirks PB, Becker LE (1994): Effects of antisense glial fibrillary acidic protein complementary DNA on the growth, invasion, and adhesion of human astrocytoma cells. Cancer Res $\underline{54}$ (12), 3267-3272 
Sallinen PK, Haapasolo HK, Visakorpi T, Helen PT, Rantala IS, Isola JJ, Helin HJ (1994): Prognostication of astrocytoma patient survival by Ki-67 (MIB-1), PCNA, and S-phase fraction using archival paraffin-embedded samples. J Pathol 174 (4), $275-282$

Sanai N, Berger MS (2008): Glioma extent of resection and its impact on patient outcome. Neurosurgery 62 (4), 753-764

Sanai N, Polley MY, McDermott MW, Parsa AT, Berger MS (2011): An extent of resection threshold for newly diagnosed glioblastomas. J Neurosurg 115 (1), 3-8

Sandler A, Gray R, Perry MC, Brahmer J, Schiller JH, Dowlati A, Lilenbaum R, Johnson DH (2006): Paclitaxel-carboplatin alone or with bevacizumab for non-small-cell lung cancer. N Engl J Med 355 (24), 2542-2550

Sartori MT, Della Puppa A, Ballin A, Saggiorato G, Bernardi D, Padoan A, Scienza R, d'Avella D, Cella G (2011): Prothrombotic state in glioblastoma multiforme: an evaluation of the procoagulant activity of circulating microparticles. J Neurooncol 104 (1), 225-231

Scherer HJ (1940): The forms of growth in gliomas and their practical significance. Brain 63 (1), 1-35

Scherer HJ (1940): Cerebral Astrocytoma and Their Derivatives. Am J Cancer 40 (2), 159-198

Schinojima N, Kochi M, Hamada J, Nakamura A, Yano S, Makino K, Tsuiki H, Tada K, Kuratsu J, Ishimaru Y (2004): The influence of sex and the presence of giant cells on postoperative long-term survival in adult patients with supratentorial glioblastoma multiforme. J Neurosur 101 (2), 219-226

Schlüter C, Duchrow M, Wohlenberg C, Becker MH, Key G, Flad HD, Gerdes J (1993): The cell proliferation-associated antigen of antibody Ki-67: a very large, ubiquitous nuclear protein with numerous repeated elements, representing a new kind of cell cycle-maintaining proteins. J Cell Biol 123 (3), 513-522

Schmidt MC, Antweiler S, Urban N, Mueller W, Kuklik A, Meyer-Puttlitz B, Wiestler OD, Louis DN, Fimmers R, von Deimling A (2002): Impact of genotype and morphology on the prognosis of glioblastoma. J Neuropathol Exp Neurol 61 (4), 321-328

Schneider T, Mawrin C, Scherlach C, Skalej M, Firsching R (2010): Gliomas in Adults. Dtsch Arztbl Int 107 (45), 799-808 
Scholzen T, Gerdes J (2000): The Ki-67 protein: from the known and the unknown. J Cell Physiol 182 (3), 311-322

Scott JG, Suh JH, Elson P, Barnett GH, Vogelbaum MA, Peereboom PM, Stevens GH, Elinzano H, Chao ST (2011): Aggressive treatment is appropriate for glioblastoma multiforme patients 70 years old or older: a retrospective review of 206 cases. Neuro-oncology 13 (4), 428-436

Scott JN, Rewcastle NB, Brashe PM, Fulton D, Hagen NA, MacKinnon JA, Sutherland G, Cairncross JG, Forsyth P (1998): Long-term glioblastoma multiforme survivors: a population-based study. Can J Neurol Sci 25 (3), 197-201

Seidel C, Dorner N, Osswald M, Wick A, Platten M, Bendzus M, Wick W (2011): Does age matter? - A MRI study on peritumoral edema in newly diagnosed primary glioblastoma. BMC Cancer $\underline{11}, 127$

Shibata S (1989): Ultrastructure of capillary walls in human brain tumors. Acta Neuropathol $\underline{78}$ (6), 561-571

Silbergeld DL, Rostomily RC, Alvord EC (1991): The cause of death in patients with glioblastoma is multifactorial: clinical factors and autopsy findings in 117 cases of supratentorial glioblastoma in adults. J Neurooncol 10 (2), 179-185

Simpson JR, Horton J, Scott C, Curran WJ, Rubin P, Fischbach J, Isaacson S, Rotman M, Asbell SO, Nelson JS (1993): Influence of location and extent of surgical resection on survival of patients with glioblastoma multiforme: results of three consecutive Radiation Therapy Oncology Group (RTOG) clinical trials. Int J Radiat Oncol Biol Phys 26 (2), 239-244

Singh SK, Hawkins C, Clarke ID, Squire JA, Bayani J, Hide T, Henkelman RM, Cusimano MD, Dirks PB (2004): Identification of human brain tumour initiating cells. Nature $\underline{432}$ (7015), 396-401

Smith ME, Eng LF (1987): Glial fibrillary acidic protein in chronic relapsing experimental allergic encephalomyelitis in SJL/J mice. J Neurosci Res 18 (1), 203-208

Smith DR, Hardman JM, Earle KM (1969): Contiguous glioblastoma multiforme and fibrosarcoma with extracranial metastasis. Cancer 24 (2), 270-276 
Smith JS, Perry AE, Borell TJ, Lee HK, O'Fallon J, Hosek SM, Kimmel D, Yates A, Burger PC, Scheithauer BW, Jenkins RB (2000): Alterations of Chromosome Arms $1 \mathrm{p}$ and $19 \mathrm{q}$ as Predictors of Survival in Oligodendrogliomas, Astrocytomas, and Mixed Oligoastrocytomas. JCO 18 (3), 636-645

Smith JS, Tachibana I, Passe SM, Huntley BK, Borell TJ, Iturria N, O'Fallon JR, Schaeffer PL, Scheithauer BW, James CD, Buckner JC, Jenkins RB (2001): PTEN mutation, EGFR amplification, and outcome in patients with anaplastic astrocytoma and glioblastoma multiforme. J Natl Cancer Inst 93 (16), 1246-1256

Souhami L, Seiferheld W, Brachman W, Podgorsak EB, Werner-Wasik M, Lustig R, Schultz CJ, Sause W, Okunieff P, Buckner J (2004): Randomized comparison of stereotactic radiosurgery followed by conventional radiotherapy with carmustine to conventional radiotherapy with carmustine for patients with glioblastoma multiforme: report of Radiation Therapy Oncology Group 93-05 protocol. Int J Radiat Oncol Biol Phys 60 (3), 853-860

Stevens GH (2006): Antiepileptic therapy in patients with central nervous system malignancies. Curr Neurol Neurosci Rep $\underline{6}$ (4), 311-318

Streffer JR, Bitzer M, Schabet M, Dichgans J, Weller M (2001): Response of radiochemotherapy-associated cerebral edema to a phytotherapeutic agent, H15. Neurology 56 (9), 1219-1221

Strugar JG, Criscuolo GR, Rothbart D, Harrington WN (1995): Vascular endothelial growth/permeability factor expression in human glioma specimens: correlation with vasogenic brain edema and tumor-associated cysts. J Neurosurg 83 (4), 682-689

Stummer W, Novotyny A, Stepp H, Goetz C, Bise K, Reulen HJ (2000):

Fluorescence-guided resection of glioblastoma multiforme by using 5-aminolevulinic acid-induced porphyrins: a prospective study in 52 consecutive patients. J Neurosurg 93 (6), 1003-1013

Stummer W, Pichlmeier U, Meine T, Wiestler OD, Zanella F, Reulen HJ (2006): Fluorescence-guided surgery with 5-aminolevulinic acid for resection of malignant glioma: a randomised controlled multicentre phase III trial. Lancet Oncol $\underline{7}$ (5), 392-401

Stupp R, Mason WP, van den Bent MJ, Weller M, Fisher B, Taphoorn M, Belanger K, Brandes AA, Marosi C, Bogdahn U (2005): Radiotherapy plus Concomitant and Adjuvant Temozolomide for Glioblastoma. N Engl J Med 352 (10), 987-996 
Stupp R, Hegi MA, Bent MJ, Mason WP, Weller M, Mirimanoff RO, Cairncross JG (2006): Changing Paradigms - An Update on the Multidisciplinary Management of Malignant Glioma. The Oncologist 11 (2), 165-180

Sun Y, Meijer DH, Albeta JA, MehtaS, Kane MF, Tien AC, Fu H, Petryniak MA, Potter GB, Liu Z (2011): Phosphorylation state of Olig2 regulates proliferation of neural progenitors. Neuron 69 (5), 906-917

Takebayashi H, Yoshida S, Sugimori M, Kosako H, Kominami R, Nakafuku M, Nabeshima Y (2000): Dynamic expression of basic helix-loop-helix Olig family members: implication of Olig2 in neuron and oligodendrocyte differentiation and identification of a new member, Olig3. Mec Dev 99 (1-2), 143-148

Taphoorn MJ, Klein M (2004): Cognitive deficits in adult patients with brain tumours. Lancet Neurol $\underline{3}$ (3), 159-168

Thaler J, Ay C, Pabinger I (2012): Clinical significance of circulating microparticles for venous thromboembolism in cancer patients. Hamostaseologie $\underline{32}$ (2), 127-131

The Cancer Genome Atlas (TCGA) Research Network (2008): Comprehensive genomic characterization defines human glioblastoma genes and core pathways. Nature $\underline{455}$ (7216), 1061-1068

Tremont-Lukats IW, Ratilal BO, Armstrong T, Gilbert MR (2008): Antiepileptic drugs for preventing seizures in people with brain tumors. Cochrane Database Syst Rev 2, CD004424

Trepant AL, Bouchart C, Rorive S, Sauvage S, Decaestecker C, Demetter P, Salmon I (2014): Identification of OLIG2 as the most specific glioblastoma stem cell marker starting from comparative analysis of data from similar DNA chip microarray platforms. Tumour biol 36 (3), 1943-1953

Turola MC, Schivalocchi R, Ramponi V, De Vito A, Nanni MG, Frivoli GF (2009): A rare case of multicentric synchronous bi-frontal glioma in a young female.

Diagnostic and therapeutic problems: a case report. Cases J 2 , 81

van den Bent MJ, Carpentier AF, Brandes AA, Sanson M, Taphoorn MJ, Bernsen HJ, Frenay M, Tijssen CC, Grisold W, Sipos L (2006): Adjuvant procarbazine, lomustine, and vincristine improves progression-free survival but not overall survival in newly diagnosed anaplastic oligodendrogliomas and oligoastrocytomas: a randomized European Organisation for Research and Treatment of Cancer phase III trial. J Clin Oncol 24 (18), 2715-2722 
Vantonen S, Timonen U, Toivanen P, Kalimo H, Kivipelto L, Heiskanen O, Unsgaard G, Kuurne T (1997): Interstitial chemotherapy with carmustine-loaded polymers for high-grade gliomas: a randomized double-blind study. Neurosurgery 41 (1), 44-49

Vaquero J, Zurita M, Morales C, Oya S, Coca S (2000): Prognostic significance of endothelial surface score and MIB-1 labeling index in glioblastoma. J Neurooncol 46 (1), 11-16

Verger E, Valduvieco I, Caral LI, Pujol T, Ribalta T, Vinolas N, Boget T, Oleaga L, Blanco Y, Graus F (2011): Does gender matter in glioblastoma? Clin Transl Oncol 13 (10), 737-741

Verhaak RG, Hoadley KA, Purdom E, Wang V, Qi Y, Wilkerosn MD, Miller CR, Ding L, Golub T, Mesirov JP (2010): Integrated genomic analysis identifies clinically relevant subtypes of glioblastoma characterized by abnormalities in PDGFRA, IDH1, EGFR, and NF1. Cancer Cell 17 (1), 98-110

Villa S, Vinolas N, Verger E, Vaya R, Martinez A, Gil M, Moreno V, Caral L, Graus F (1998): Efficacy of radiotherapy for malignant gliomas in elderly patients. Int J Radiat Oncol Biol Phys 42 (5), 977-980

Vordermark D, Ruprecht K, Rieckmann P, Roggendorf W, Vince GH, Warmuth-Metz M, Klbl O, Flentje M (2006): Glioblastoma multiforme with oligodendroglial component (GBMO): favorable outcome after post-operative radiotherapy and chemotherapy with nimustine (ACNU) and teniposide (VM26). BMC Cancer $\underline{6}, 247$

Vousden KH, Prives C (2009): Blinded by the Light: The Growing Complexity of p53. Cell $\underline{137}(3), 413-431$

Wang Y, Li S, Chen L, You G, Bao Z, Yan W, Shi Z, Chen Y, Yao K, Zhang W (2012): Glioblastoma with an oligodendroglioma component: distinct clinical behavior, genetic alterations, and outcome. Neuro-oncology 14 (4), 518-525

Watanabe K, Tachibana O, Sata K, Yonekawa Y, Kleihues P, Ohgaki H (1996): Overexpression of the EGF receptor and p53 mutations are mutually exclusive in the evolution of primary and secondary glioblastomas. Brain Pathol $\underline{6}$ (3), 217-123

Watts GS, Pieper RO, Costello JF, Peng YM, Dalton WS, Futscher BW (1997): Methylation of discrete regions of the O6-methylguanine DNA methyltransferase (MGMT) CpG island is associated with heterochromatinization of the MGMT transcription start site and silencing of the gene. Mol Cell Biol 17 (9), 5612-5619 
Wei P, Zhang W, Yang LS, Zhang HS, Xu XE, Jiang YH, Huang FP, Shi Q (2013): Serum GFAP autoantibody as an ELISA-detectable glioma marker. Tumour Biol 34 (4), 2283-2292

Weinstein DE, Shelanski ML, Liem RK (1991): Suppression by antisense mRNA demonstrates a requirement for the glial fibrillary acidic protein in the formation of stable astrocytic processes in response to neurons. J Cell Biol 112 (6), 1205-1213

Weller M, Wick W (2011): Are we ready to demystify age in glioblastoma? Or does older age matter in glioblastoma? Neuro-oncology 13 (4), 365-366

Westphal M, Hilt DC, Bortey E, Delavault P, Olivares R, Warnke PC, Whittle IR, Jaaskelainen J, Ram J (2003): A phase 3 trial of local chemotherapy with biodegradable carmustine (BCNU) wafers (Gliadel wafers) in patients with primary malignant glioma. Neuro-oncology $\underline{5}(2), 79-88$

Westphal M, Ram Z, Riddle V, Hilt D, Bortey E (2006): Gliadel wafer in initial surgery for malignant glioma: long-term follow-up of a multicenter controlled trial. Acta Neurochir 148 (3), 269-275

Whitesell L, Lindquist SL (2005): HSP90 and the chaperoning of cancer. Nat Rev Cancer $\underline{5}(10), 761-772$

Wick W, Kueker W (2004): Brain edema in neurooncology: radiological assessment and management. Onkologie 27 (3), 261-266

Wick W, Weller M, Weiler M, Batchelor T, Yung AW, Platten M (2011): Pathway inhibition: emerging molecular targets for treating glioblastoma. Neuro Oncol 13 (6), 566-579

Wilhelmsson U, Eliasson C, Bjerkvig R, Pekny M (2003): Loss of GFAP expression in high-grade astrocytomas does not contribute to tumor development or progression. Oncogene $\underline{22}$ (22), 3407-3411

Wrensch M, Minn Y, Chew T, Bondy M, Berger MS (2002): Epidemiology of primary brain tumors: current concepts and review of the literature. Neuro Oncol $\underline{4}$ (4), 278-299

Xiong NX, Zhao HY, Zhang FC, He ZQ (2007): Negative correlation of Nogo-A with the malignancy of oligodendroglial tumor. Neurosci Bull 23 (1), 41-45

Yahanda AM, Bruner JM, Donehower LA, Morrison RS (1995): Astrocytes derived from p53-deficient mice provide a multistep in vitro model for development of malignant gliomas. Mol Cell Biol 15 (8), 4249-4259 
Yan H, Parsons DW, Jin G, McLendon R, Rasheed BA, Yuan W, Kos I, Batinic-Haberle I, Jones S, Riggins GJ (2009): IDH1 and IDH2 mutations in gliomas. N Engl J Med 360 (8), 765-773

Yang JC, Haworth L, Sherry RM , Hwu P, Schwartzentruber DJ, Topalian SL, Steinberg SM, Chen HX, Rosenberg SA (2003): A randomized trial of bevacizumab, an anti-vascular endothelial growth factor antibody, for metastatic renal cancer. N Engl J Med 349 (5), 427-434

Yong RL, Lonser RR (2011): Surgery for glioblastoma multiforme: striking a balance. World Neurosurgery 76 (6), 528-530

Yoshimoto K, Mizoguchi M, Hata N, Murata H, Hatae R, Amano T, Nakamizo A, Sasaki T (2012): Complex DNA repair pathways as possible therapeutic targets to overcome temozolomide resistance in glioblastoma. Front. Oncol 2 186, 1-8

Yu L, Qi ST, Li ZY (2010): Analysis of isocitrate dehydrogenase-1/2 gene mutations in gliomas. Chin Med J 123 (24), 3697-3705

Zhou Q, Anderson DJ (2002): The bHLH transcription factors OLIG2 and OLIG1 couple neuronal and glial subtype specification. Cell 109 (1), 61-73

Zhou Q, Wang S, Anderson DJ (2000): Identification of a novel family of oligodendrocyte lineage-specific basic helix-loop-helix transcription factors. Neuron 25 (2), 331-343

Zou P, Xu H, Chen P, Yan Q, Zhao L, Zhao P, Gu A (2013): IDH1/IDH2 mutations define the prognosis and molecular profiles of patients with gliomas: a meta-analysis. PLos One 8 (7), e68782. 


\section{Acknowledgements}

First and foremost, I would like to express my immense gratitude to Professor Christine Stadelmann and Doctor Alonso Barrantes-Freer of the Department of Neuropatholgy of the University Medical Center Göttingen. Without their enormous support this study would not have been possible. The numerous meetings, chats in the hallway and e-mails helped me keep on schedule with my workload and to further develop my scientific thinking. I am very grateful for all that I was taught.

I am also indebted to Professor Christian Hartmann, director of the Department of Neuropathology at the Hannover Medical School, for performing the initial histopathological evaluation and marking of the tumor samples. I also wish to thank Doctor Florian Stockhammer and his doctoral students of the Department of Neurosurgery of the University Medical Center Göttingen for providing the clinical data of the patient collective necessary for the statistical analysis. The Tissue Microarray method would not have been possible without the help and support of Doctor Carl-Ludwig Behnes of the Department of Pathology of the University Medical Center Göttingen. I am also thankful for the support of Doctor Tobias Pukrop of the Department of Hematology and Oncology of the University Medical Center Göttingen. At this point I would also like to thank Professor Wolfgang Brück, director of the Department of Neuropathology of the University Medical Center Göttingen, for giving me the opportunity to undertake this research project and the doctoral thesis.

Last but not least I would like to thank the many colleagues of the Department of Neuropathology of the University Medical Center Göttingen for introducing me to the laboratory and helping me with little problems like computer access, unlocking rooms, finding materials and tissue samples.

I owe a special thanks to an undefined group of people who will probably never read this. Most of the statistical analysis, all image processing and the writing of this thesis were done with open source software (R-Studio, Gimp and Latex). Thanks to the enormous support through internet forums and free youtube tutorials I was able to learn to use these programs quickly and was able to solve many little problems and issues as promptly as they occurred. I can not find the right words to express the excitement I feel about the endless opportunities the internet offers to the scientific world and the spread of knowledge and information nowadays. 
Identification of Prognostically Relevant Cellular Markers of Differentiation in Glioblastoma 


\section{Curriculum Vitae}

I, Felix Behling, was born on the 5th of September in 1983 as the second of three children to the married couple Dipl. Ing. Volker Horst Günther Behling and Dipl. Ing. Uta Behling.

After visiting the Martin-Luther Elementary School in Northeim from 1990 to 1994, I enrolled in the Thomas-Mann Orientierungsstufe in Northeim for 2 years. In 1996 I started attending the Gymnasium Corvinianum, a grammar school in Northeim. In the school year of 2000/2001 I took part in an exchange student program organized by the Rotary Club. After attending a full school year at the High School in Caseville Michigan, I finished grammar school in 2003 receiving the higher education entrance qualification (Abitur). From October 2003 till July 2004 I fulfilled my compulsory civilian service in the Intermediate Care Unit of the Marienhospital in Stuttgart.

In October 2004 I enrolled at the University of Göttingen for the studies of agricultural sciences. Subsequently, in April 2005 I started my studies in Medical School and passed the preliminary medical examination (Physikum) in May 2007. From August 2008 until July 2009 I temporarily discontinued my clinical medical education to research the role of microRNAs in a cell culture model of Huntington disease (Department of Neurology of the University Medical Center Göttingen, Prof. Dr. Mathias Bähr). In October 2009 I continued Medical School and in my final year I chose clinical electives in the Department of Surgery at the Protestant Hospital in Göttingen, the Department of Cardiology and the Department of Neurosurgery at the University Medical Center Göttingen and the Department of Neurosurgery at the National Hospital for Neurology and Neurosurgery in London.

I passed the Medical State Examination in June 2012 and received my medical license (Approbation) on the 3rd of July in 2012. In July 2011 and 2012 I passed the first and second step of the United States Medical Licensing Examination, respectively.

In September 2012 I started the research project subject to this dissertation under the supervision of Prof. Dr. med. Christine Stadelmann at the Department of Neuropathology at the University Medical Center Göttingen.

Since the first of July 2013 I started my residency at the Department of Neurosurgery at the University Clinic Tübingen under the direction of Prof. Dr. med. Marcos Soares Tatagiba. 